\title{
Bis- $N$-Heterocyclic Carbene Complexes of Coinage Metals Containing Four Naphthalimide Units: A Structure-Emission Properties Relationship Study
}

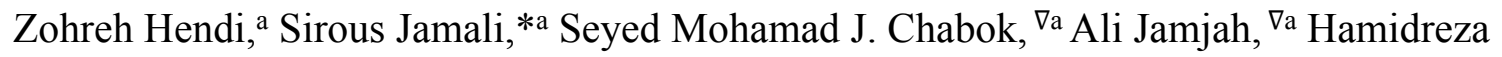 \\ Samouei, ${ }^{\mathrm{b}}$ Zahra Jamshidi ${ }^{\mathrm{a}}$ \\ a Department of Chemistry, Sharif University of Technology, P.O. Box 11155-3516, Tehran, \\ Iran \\ ${ }^{\mathrm{b}}$ Department of Chemistry, Texas A\&M University, P.O. Box 30012, College Station, Texas \\ 77842-3012, United States
}

Sirous Jamali*: sjamali@sharif.edu 


\section{Table of Content}

$1 \quad \mathrm{X}$-Ray Structure Determination.......................................... S1

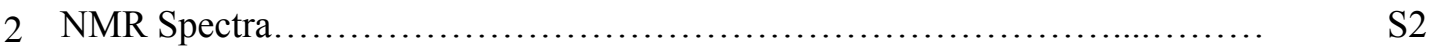

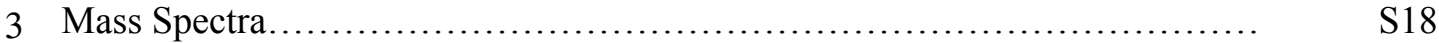

4 Crystal Structures...................................................... S20

5 Photophysical properties...........................................

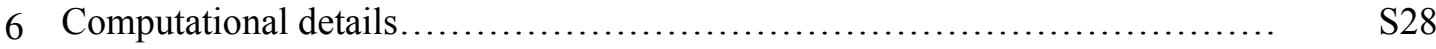

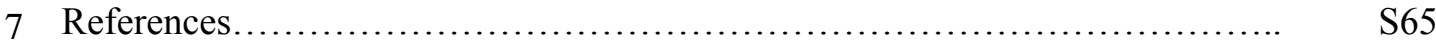




\section{X-Ray Structure Determination}

X-ray intensity data were collected using the full sphere routine by an $\omega$ scan strategy on the Agilent SuperNova dual-wavelength EoS S2 diffractometer with mirror monochromated $\mathrm{Cu}$ Ka radiation $(\lambda=1.54184 \AA)$ for 3 and 4(parallel) and the Bruker SMART Apex2CCD diffractometer with graphite monochromated Mo Ka radiation $(\lambda$ $=0.71073 \AA$ ) for 4 (twisted) and 5. The data collection were done at room temperature for all the complexes. Data reduction, including an empirical absorption correction using spherical harmonics, implemented in the SCALE3 ABSPACK scaling algorithm, ${ }^{1}$ was performed using the CrysAlisPro software package and SAINT ${ }^{2}$ for all the complexes. The crystal structures of all the complexes were determined by direct methods using the online version of AutoChem 2.0 in conjunction with the OLEX $2^{3}$ suite of programs implemented in the CrysAlis software and then refined by full-matrix least squares (SHELXL-2018) ${ }^{4}$ on F2. The non-hydrogen atoms were refined anisotropically. All of the hydrogen atoms were positioned geometrically in idealized positions and refined with the riding model approximation, with $\operatorname{Uiso}(\mathrm{H})=1.2$ or $1.5 \mathrm{Ueq}(\mathrm{C})$. All geometric calculations were carried out using the PLATON software. ${ }^{5}$ The solvent accessible voids of both structures were squeezed by the SQUEEZE ${ }^{6}$ routine in PLATON by back Fourier transform. Crystallographic data for the structural analysis have been deposited with the Cambridge Crystallographic Data Centre, No. CCDC 2070212, 2070213, 2070214, and 2070215. 


\section{NMR Spectra}
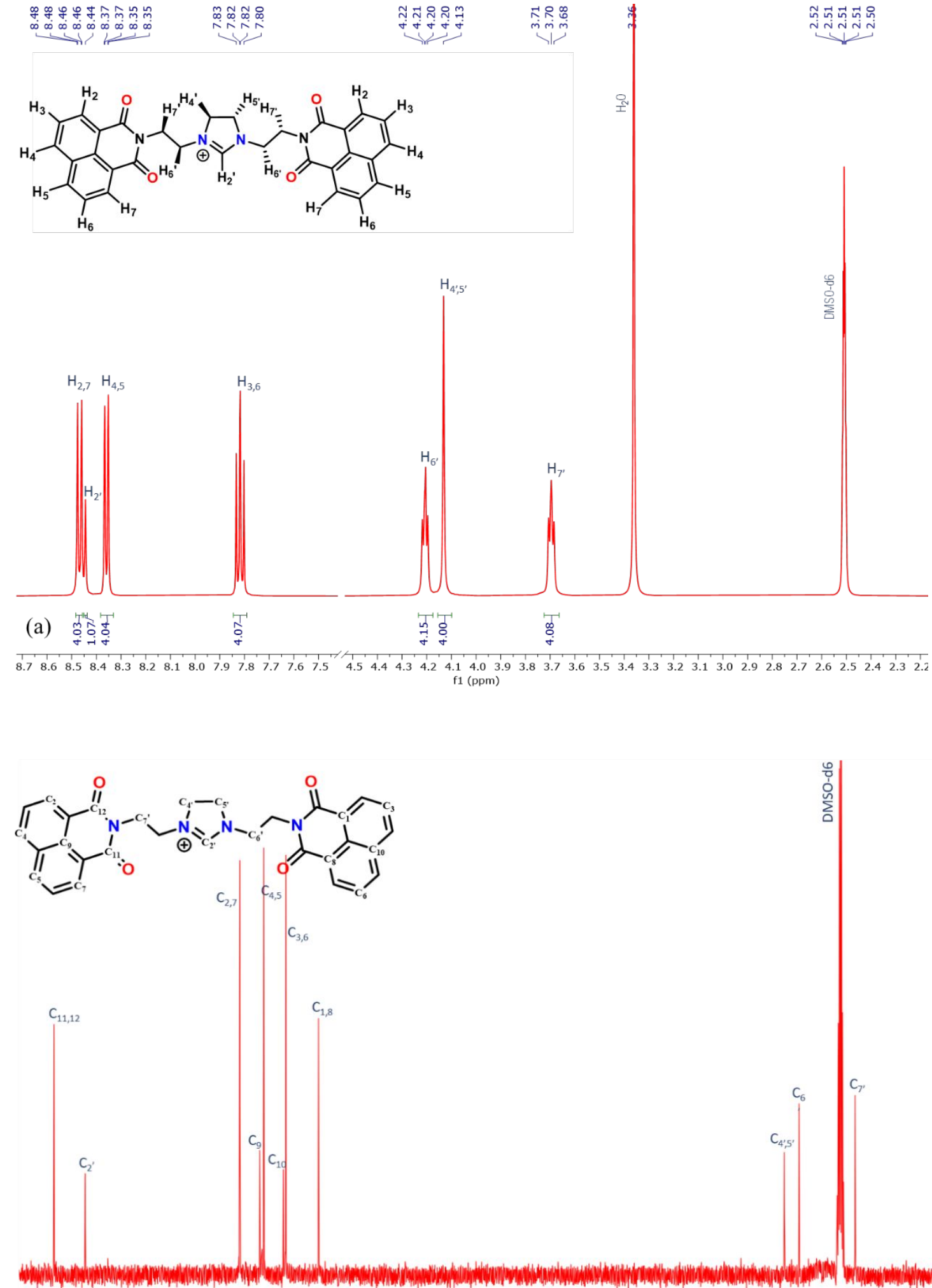

(b)

Figure S1. (a) ${ }^{1} \mathrm{H}$ NMR and (b) ${ }^{13} \mathrm{C}\left\{{ }^{1} \mathrm{H}\right\}$ NMR spectrum of 2a in DMSO- $d_{6}$ at $298 \mathrm{~K}$. 


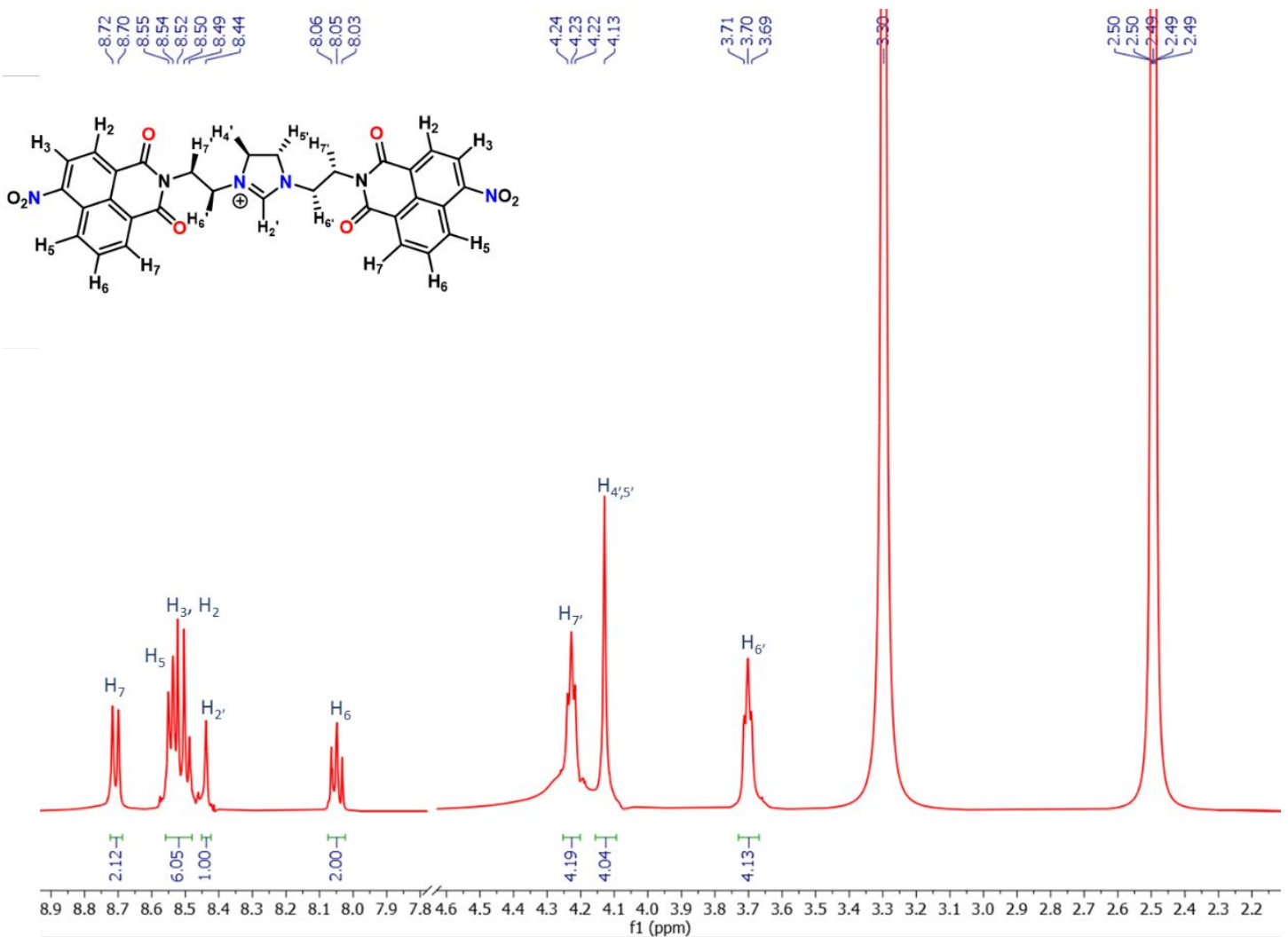

Figure S2. ${ }^{1} \mathrm{H}$ NMR spectrum of $\mathbf{2 b}$ in DMSO- $d_{6}$ at $298 \mathrm{~K}$. 


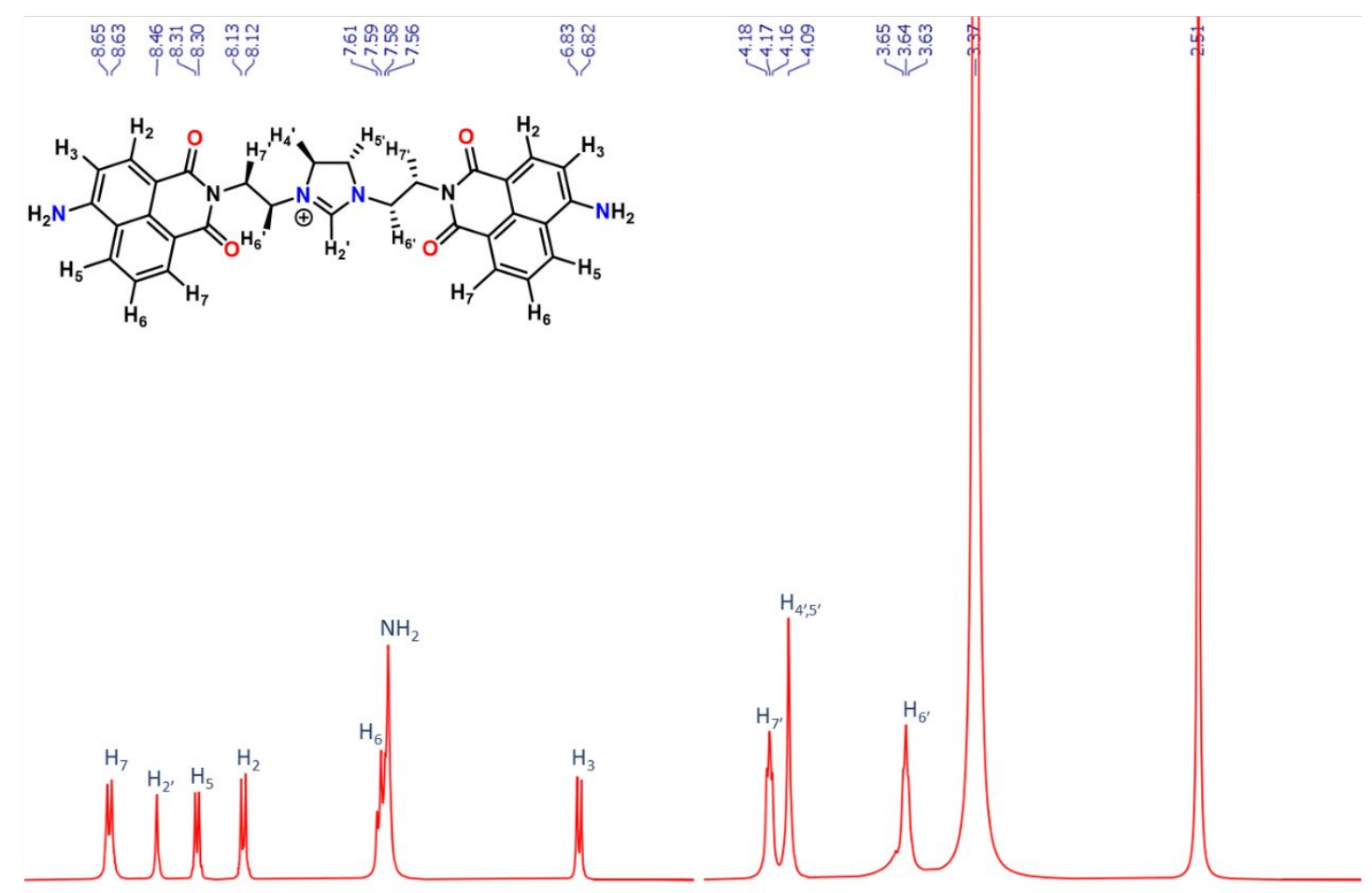

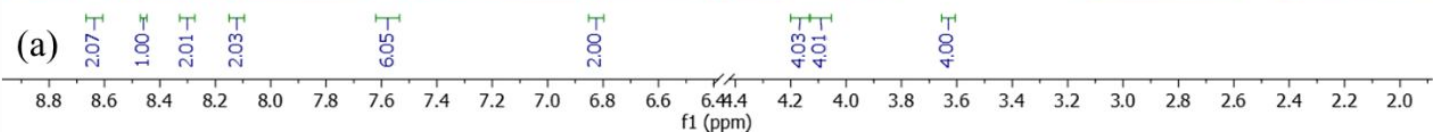

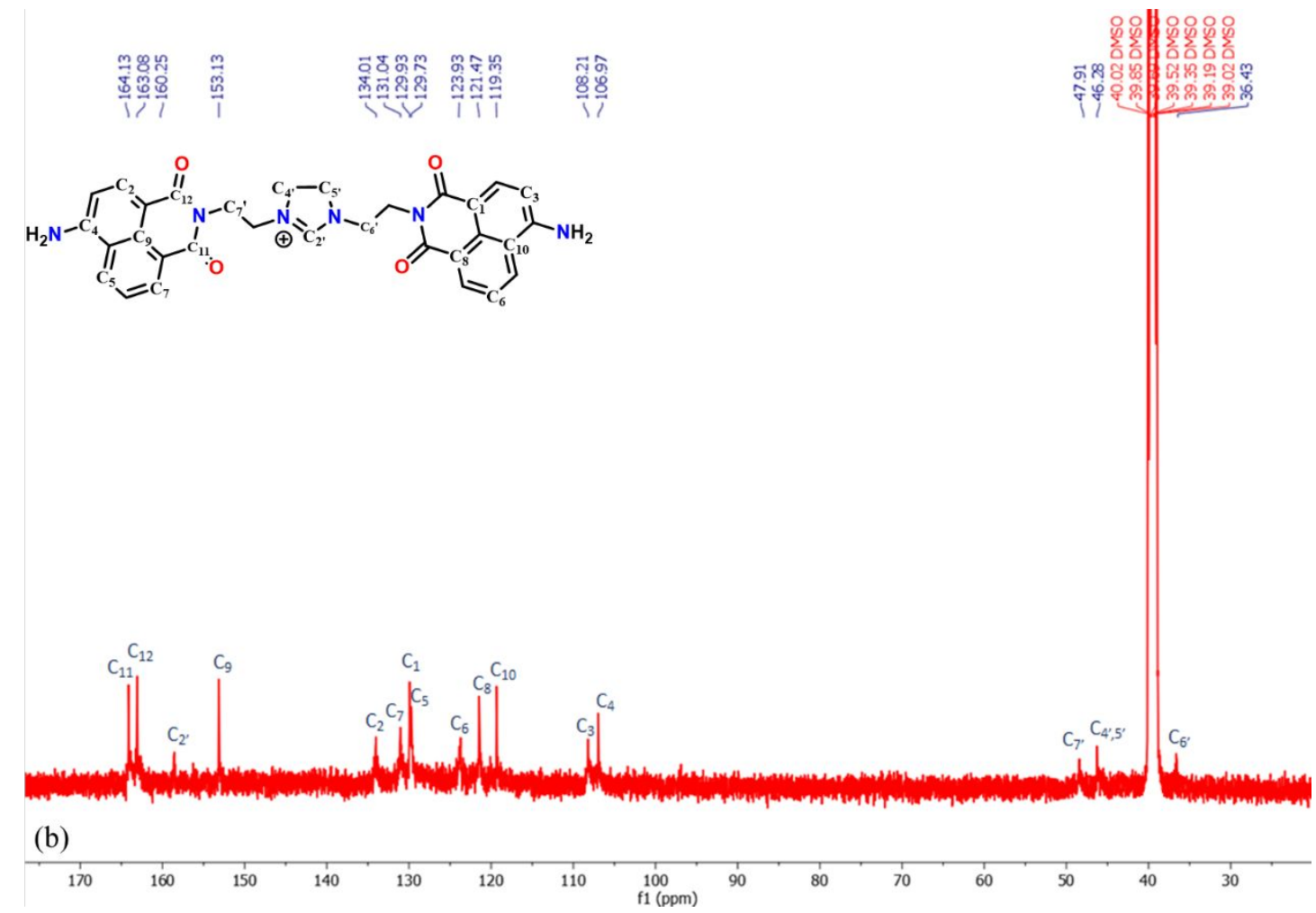

Figure S3. (a) ${ }^{1} \mathrm{H}$ NMR spectrum of 2a in DMSO- $d_{6}$ at $298 \mathrm{~K}$, (b) ${ }^{13} \mathrm{C}\left\{{ }^{1} \mathrm{H}\right\}$ NMR spectrum of 2a in DMSO- $d_{6}$ at $298 \mathrm{~K}$. 


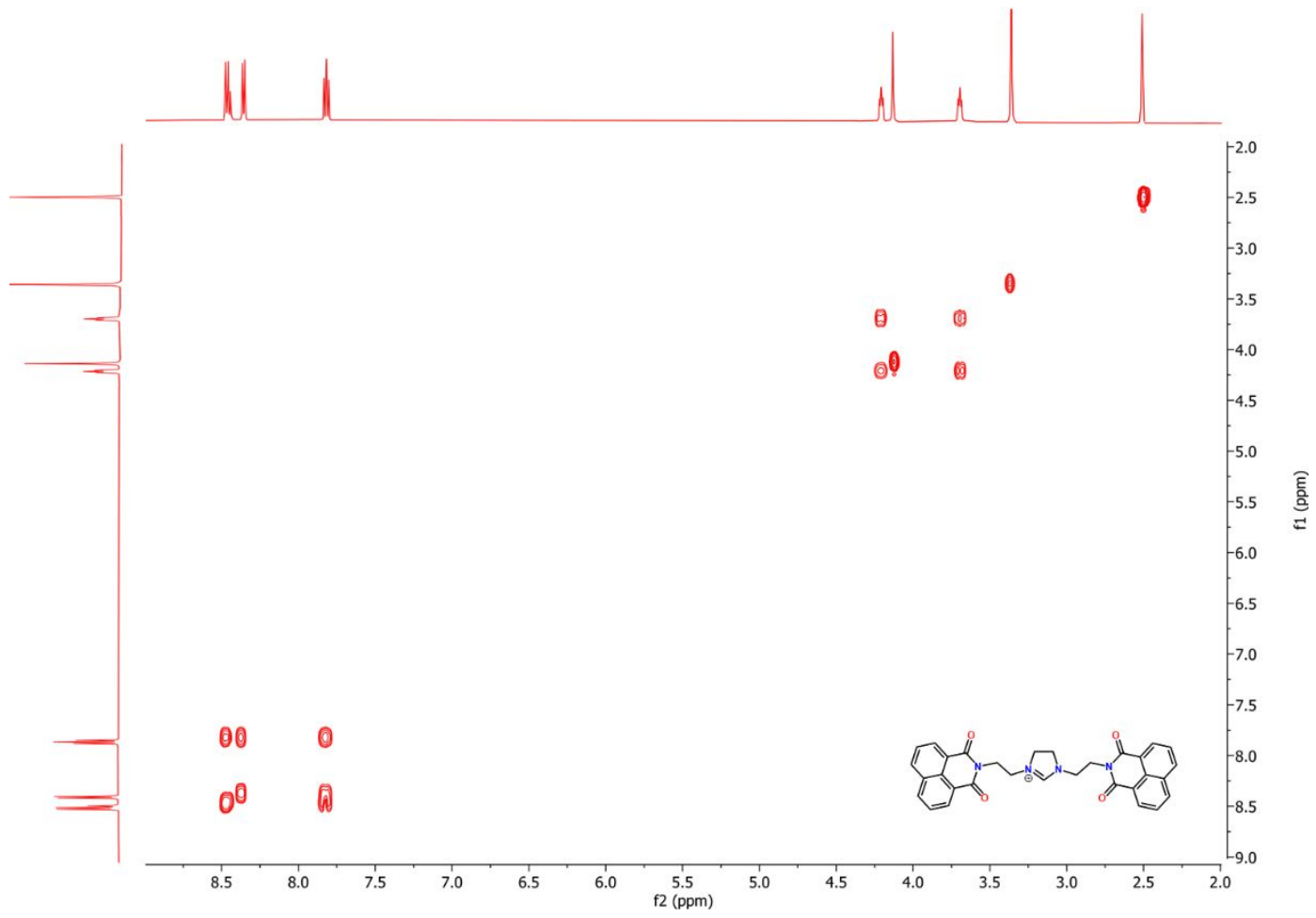

Figure $\mathbf{S 4} .{ }^{1} \mathrm{H}-{ }^{1} \mathrm{H}$ COSY NMR spectrum of $\mathbf{2 a}$ in DMSO- $d_{6}$ at $298 \mathrm{~K}$.

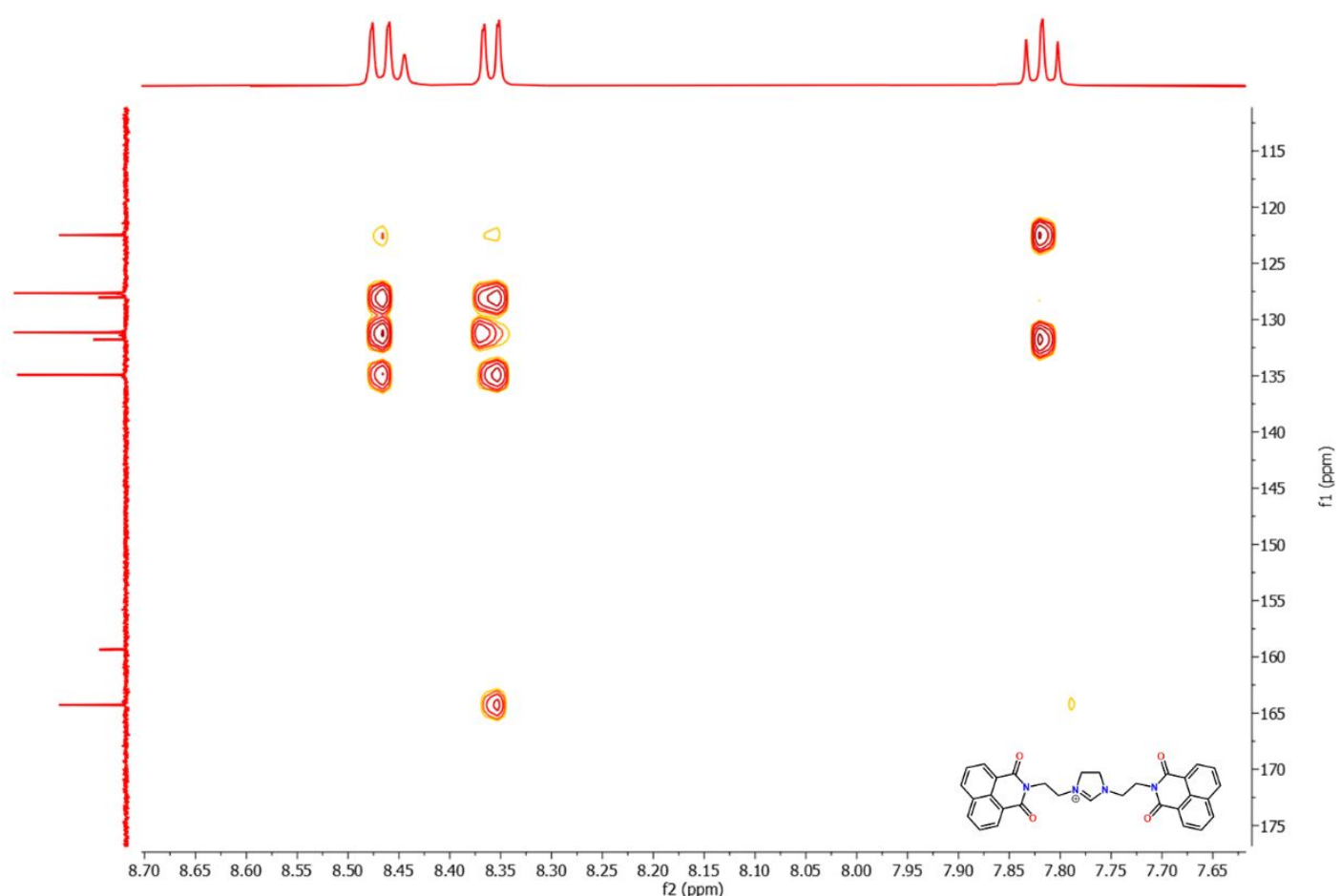

Figure S5. ${ }^{1} \mathrm{H}-{ }^{13} \mathrm{C}$ HMBC NMR spectrum of 2a in DMSO- $d_{6}$ at $298 \mathrm{~K}$. 


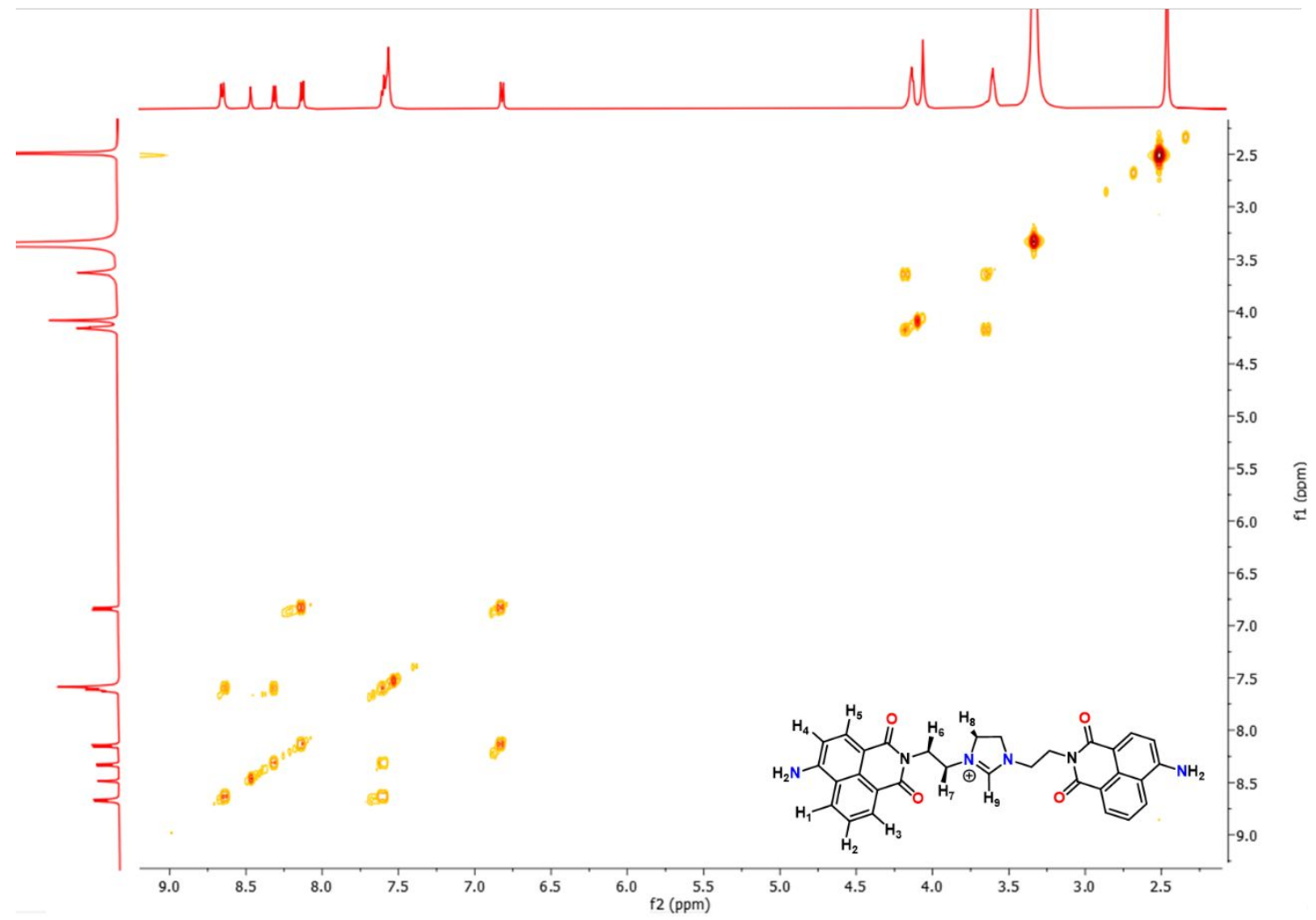

Figure S6. ${ }^{1} \mathrm{H}-{ }^{1} \mathrm{H}$ COSY NMR spectrum of $2 \mathrm{c}$ in DMSO- $d_{6}$ at $298 \mathrm{~K}$.

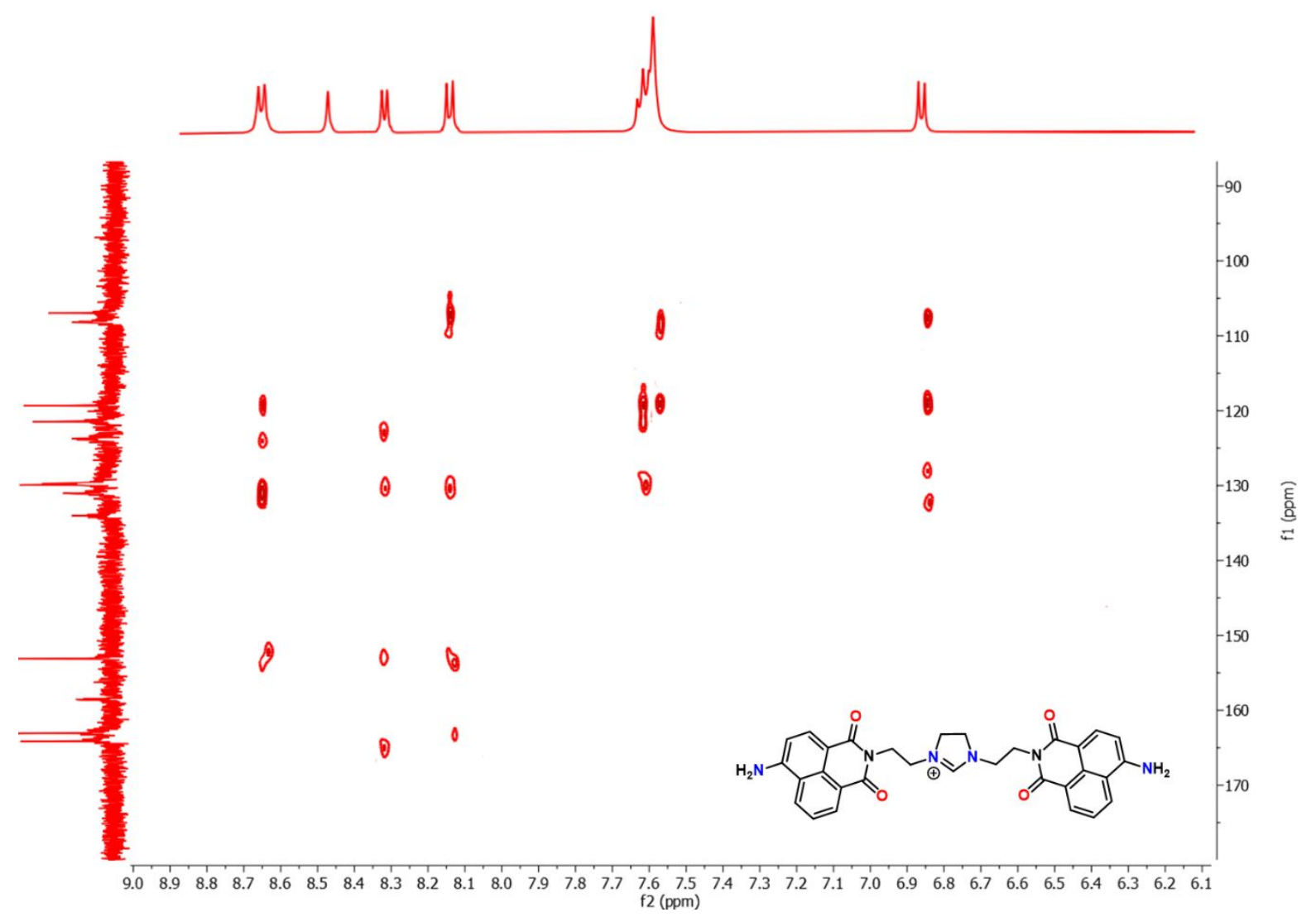

Figure S7. ${ }^{1} \mathrm{H}_{-}{ }^{13} \mathrm{C}$ HMBC NMR spectrum of $2 \mathrm{c}$ aromatic part, in DMSO- $d_{6}$ at $298 \mathrm{~K}$. 


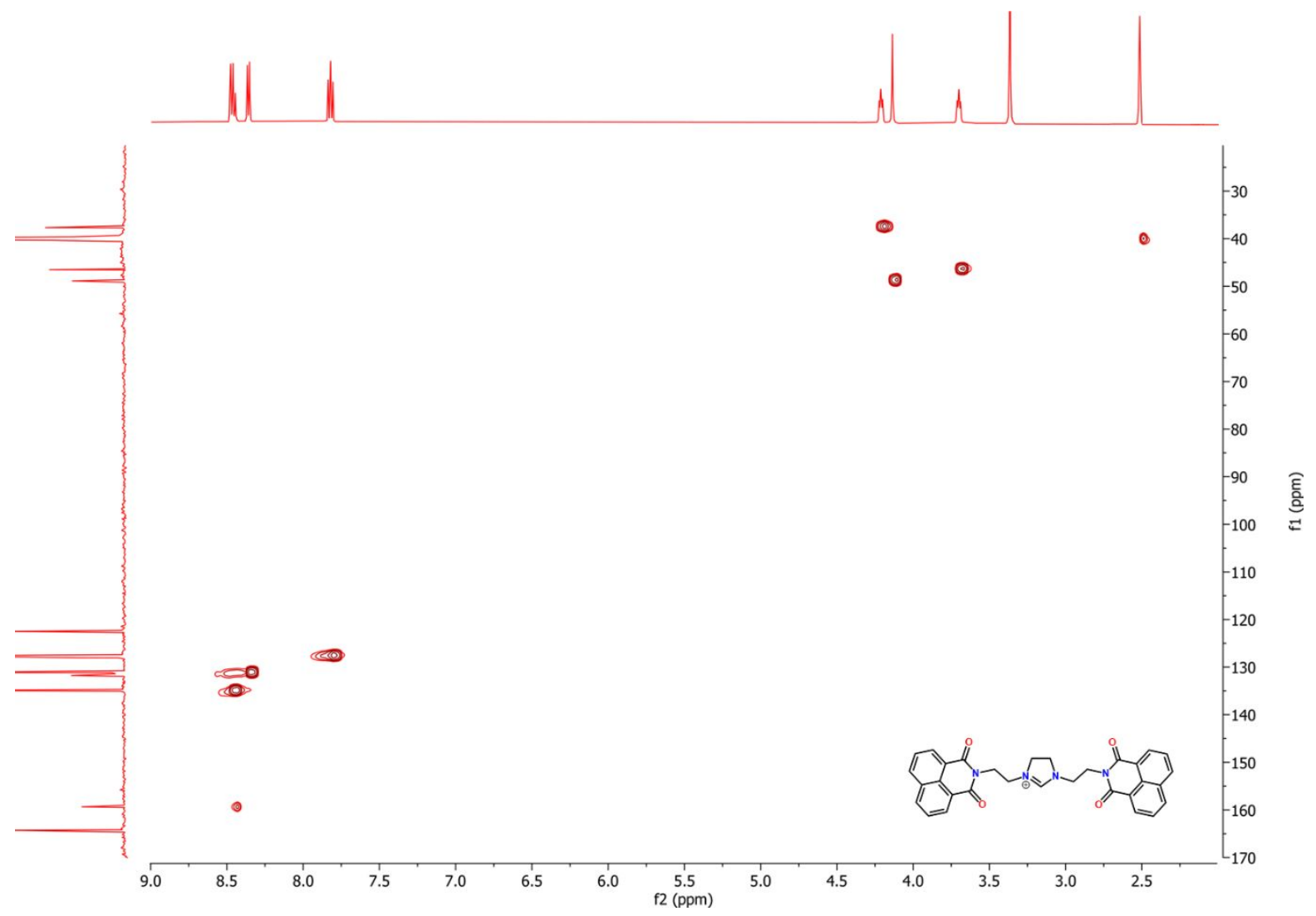

Figure S8. ${ }^{1} \mathrm{H}-{ }^{13} \mathrm{C}$ HSQC NMR spectrum of 2a in DMSO- $d_{6}$ at $298 \mathrm{~K}$.

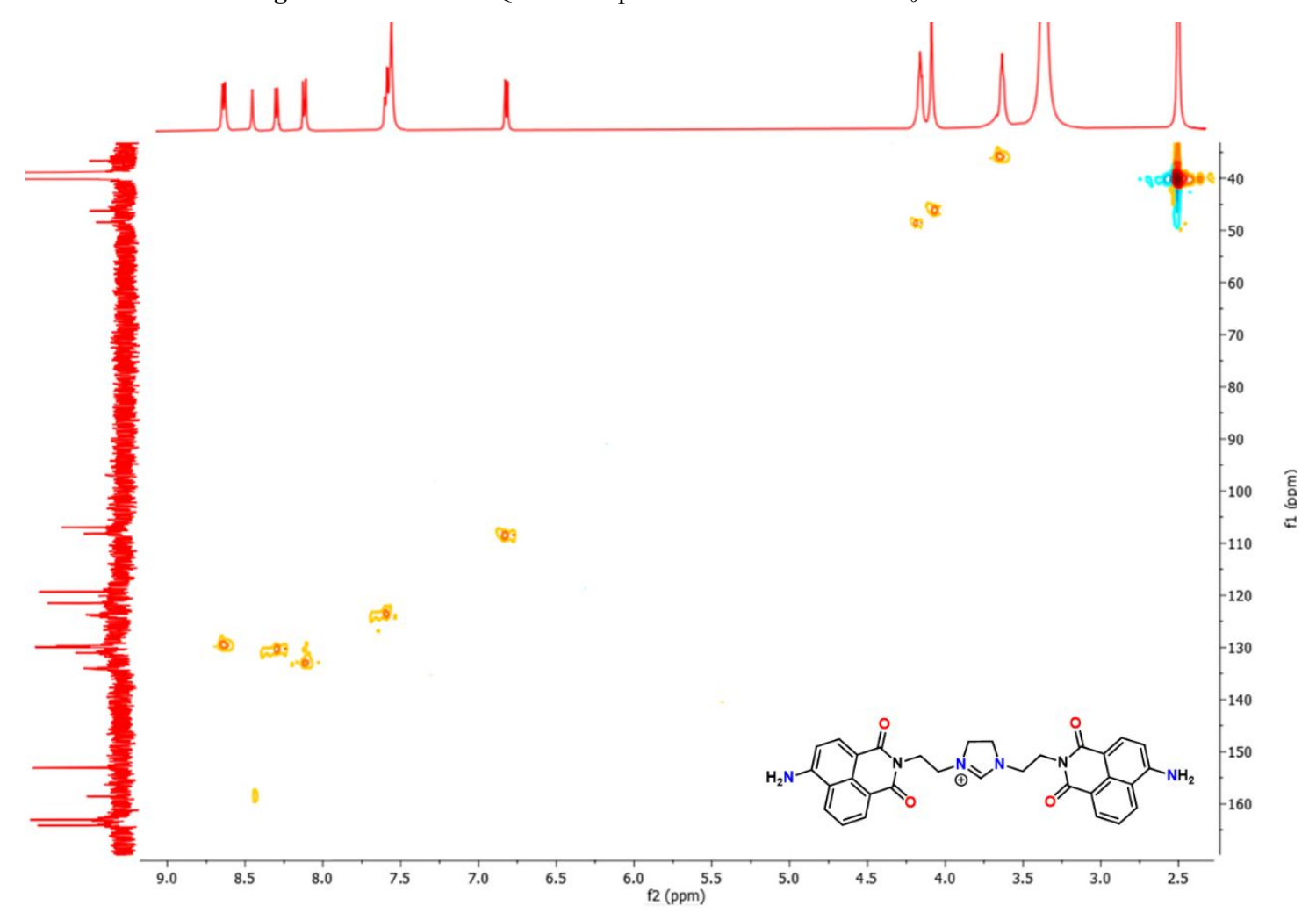

Figure S9. ${ }^{1} \mathrm{H}-{ }^{13} \mathrm{C}$ HSQC NMR spectrum of $2 \mathbf{c}$ in DMSO- $d_{6}$ at $298 \mathrm{~K}$. 


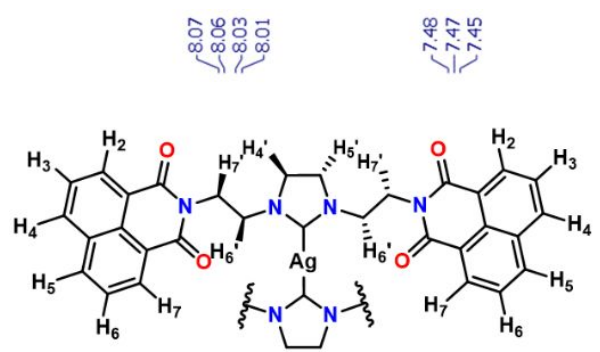

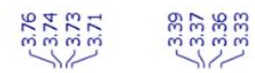
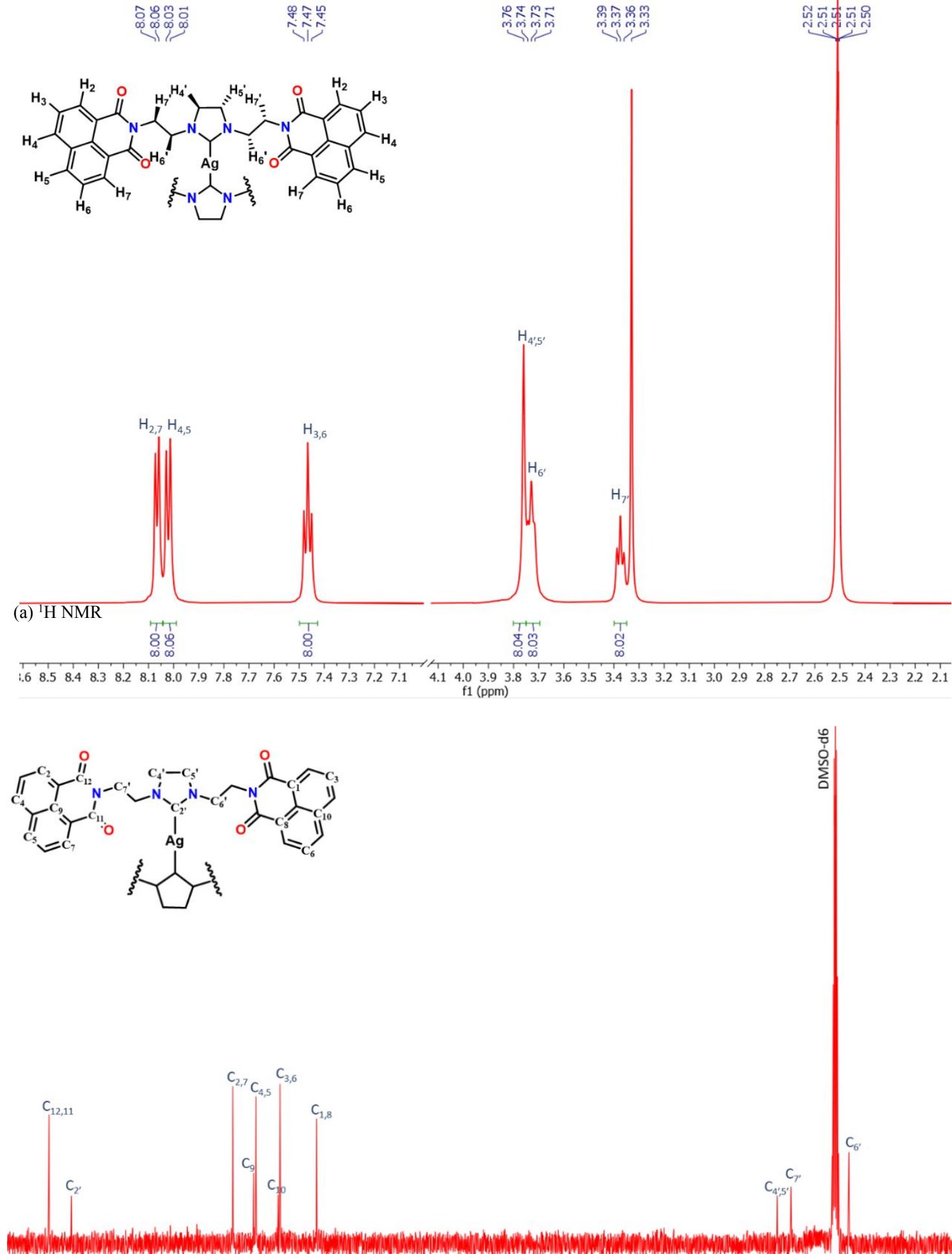

(b) ${ }^{160} \mathrm{C}\left\{{ }^{1} \mathrm{H}\right\} \mathrm{NMR}$ 

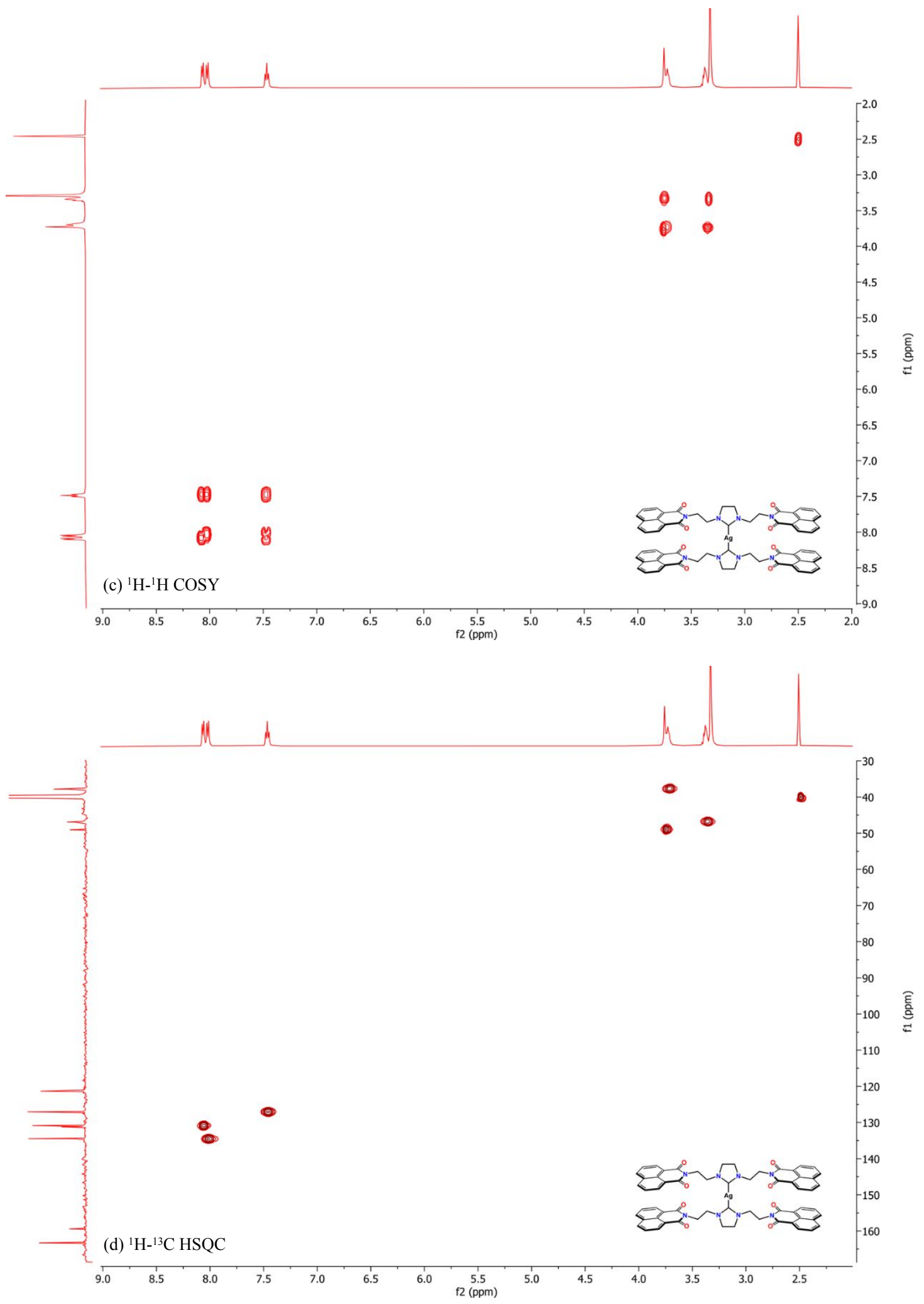


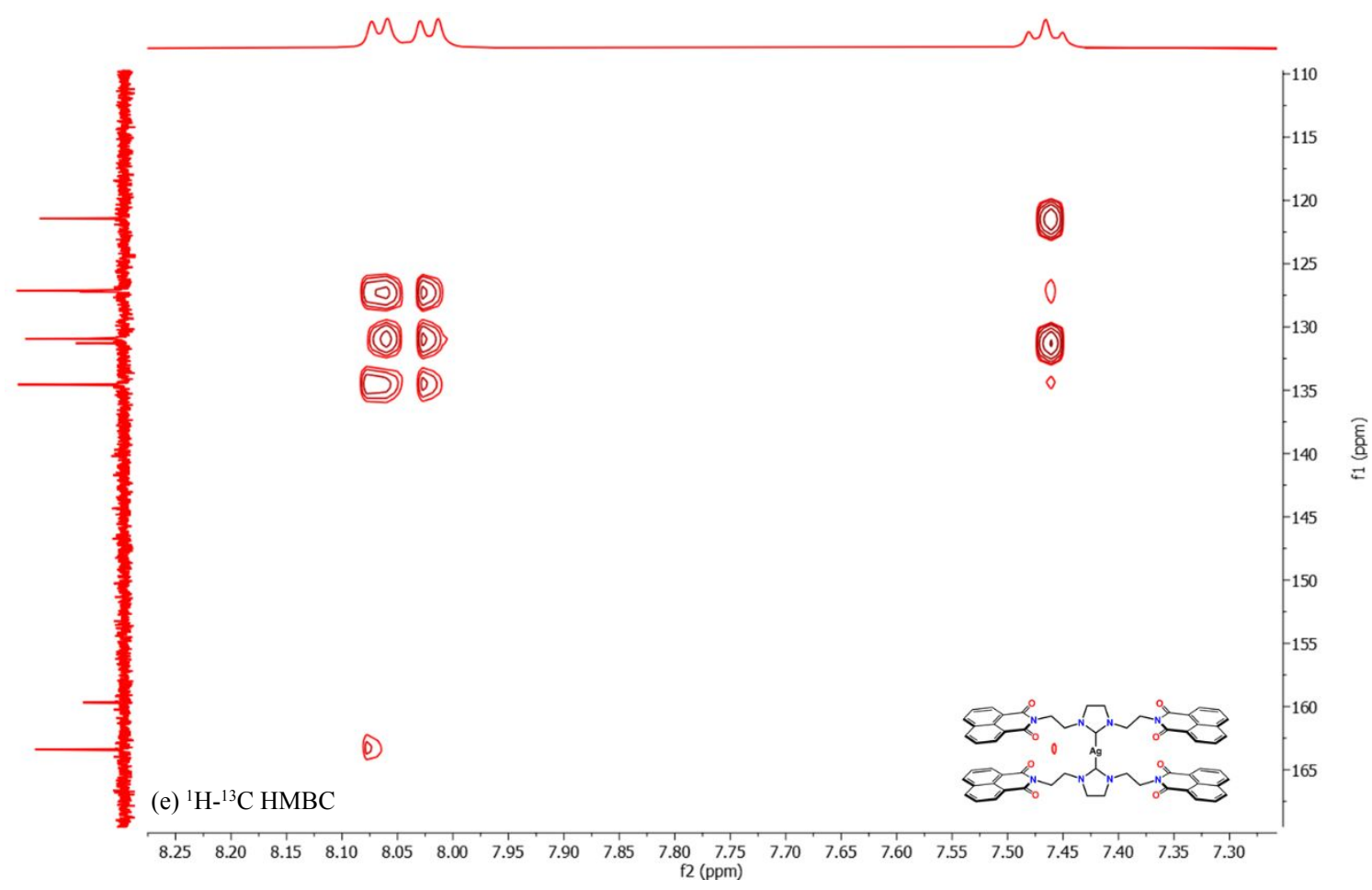

Figure S10. (a) ${ }^{1} \mathrm{H}$ NMR spectrum of 3 in DMSO- $d_{6}$ at $298 \mathrm{~K}$. (b) ${ }^{13} \mathrm{C}\left\{{ }^{1} \mathrm{H}\right\}$ NMR spectrum of $\mathbf{3}$ in DMSO- $d_{6}$ at $298 \mathrm{~K}$. (c) ${ }^{1} \mathrm{H}-{ }^{1} \mathrm{H}$ COSY NMR spectrum of 3 in DMSO- $d_{6}$ at $298 \mathrm{~K}$. (d) ${ }^{1} \mathrm{H}^{13} \mathrm{C}$ HSQC NMR spectrum of $\mathbf{3}$ in DMSO- $d_{6}$ at $298 \mathrm{~K}$. (e) ${ }^{1} \mathrm{H}-{ }^{13} \mathrm{C}$ HMBC NMR spectrum of $\mathbf{3}$, aromatic part, in DMSO- $d_{6}$ at $298 \mathrm{~K}$.
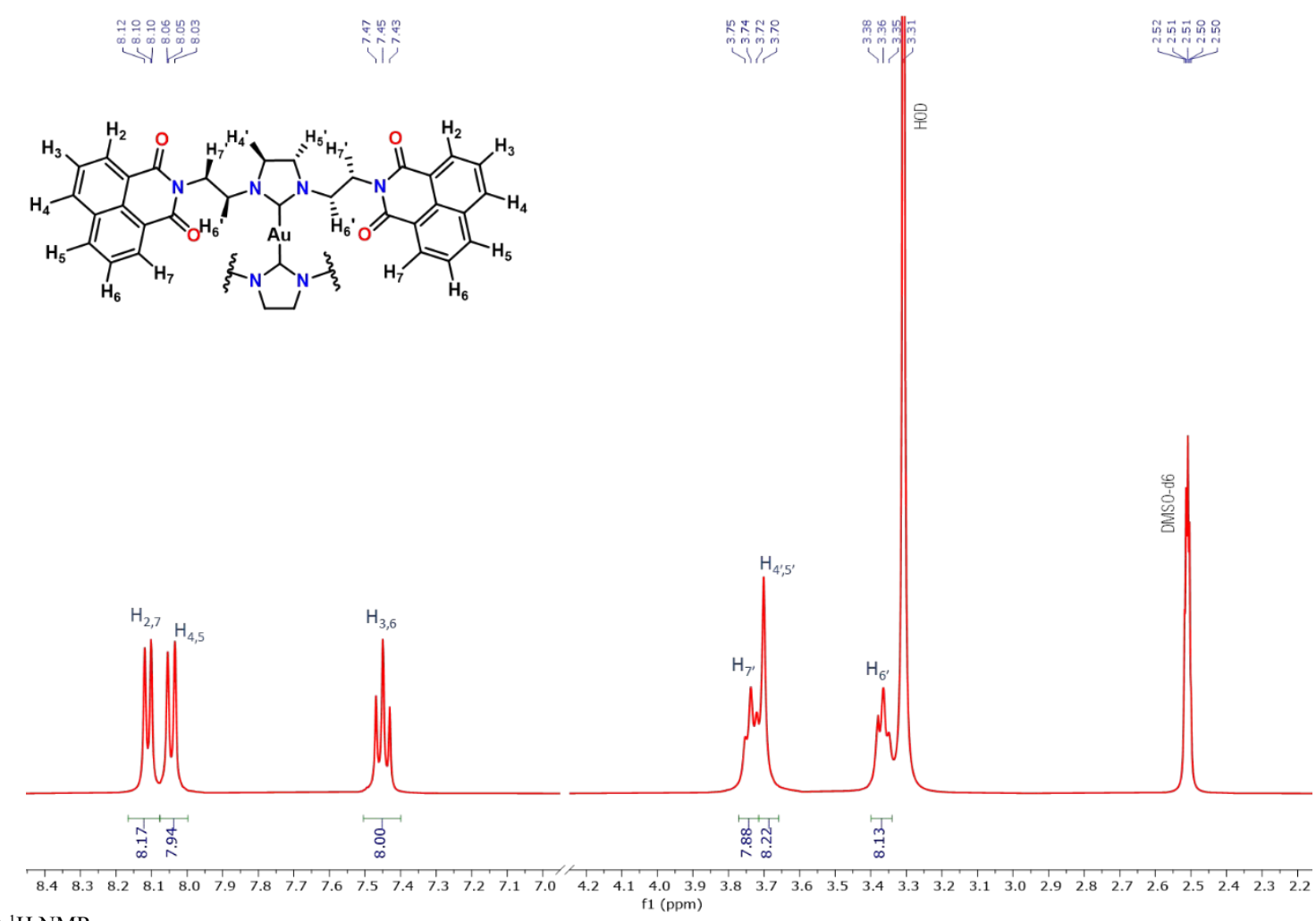

(a) ${ }^{1} \mathrm{H}$ NMR 

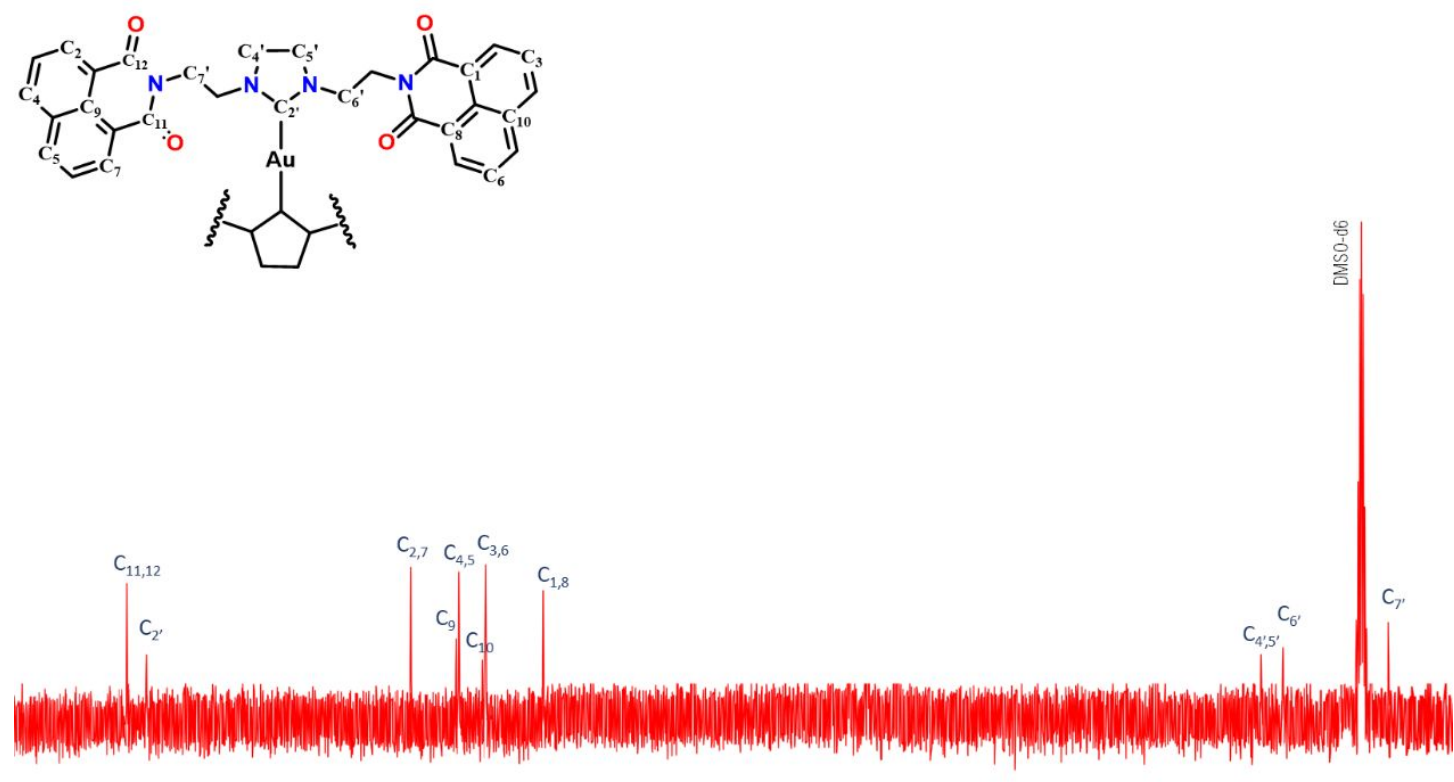

\begin{tabular}{llllllllllllllllllllllllllllllllllllllll}
\hline 170 & 165 & 160 & 155 & 150 & 145 & 140 & 135 & 130 & 125 & 120 & 115 & 110 & $\begin{array}{c}105 \\
\mathrm{f}(\mathrm{ppm})\end{array}$ & 95 & 90 & 85 & 80 & 75 & 70 & 65 & 60 & 55 & 50 & 45 & 40 & 35
\end{tabular} (b) ${ }^{13} \mathrm{C}\left\{{ }^{1} \mathrm{H}\right\} \mathrm{NMR}$

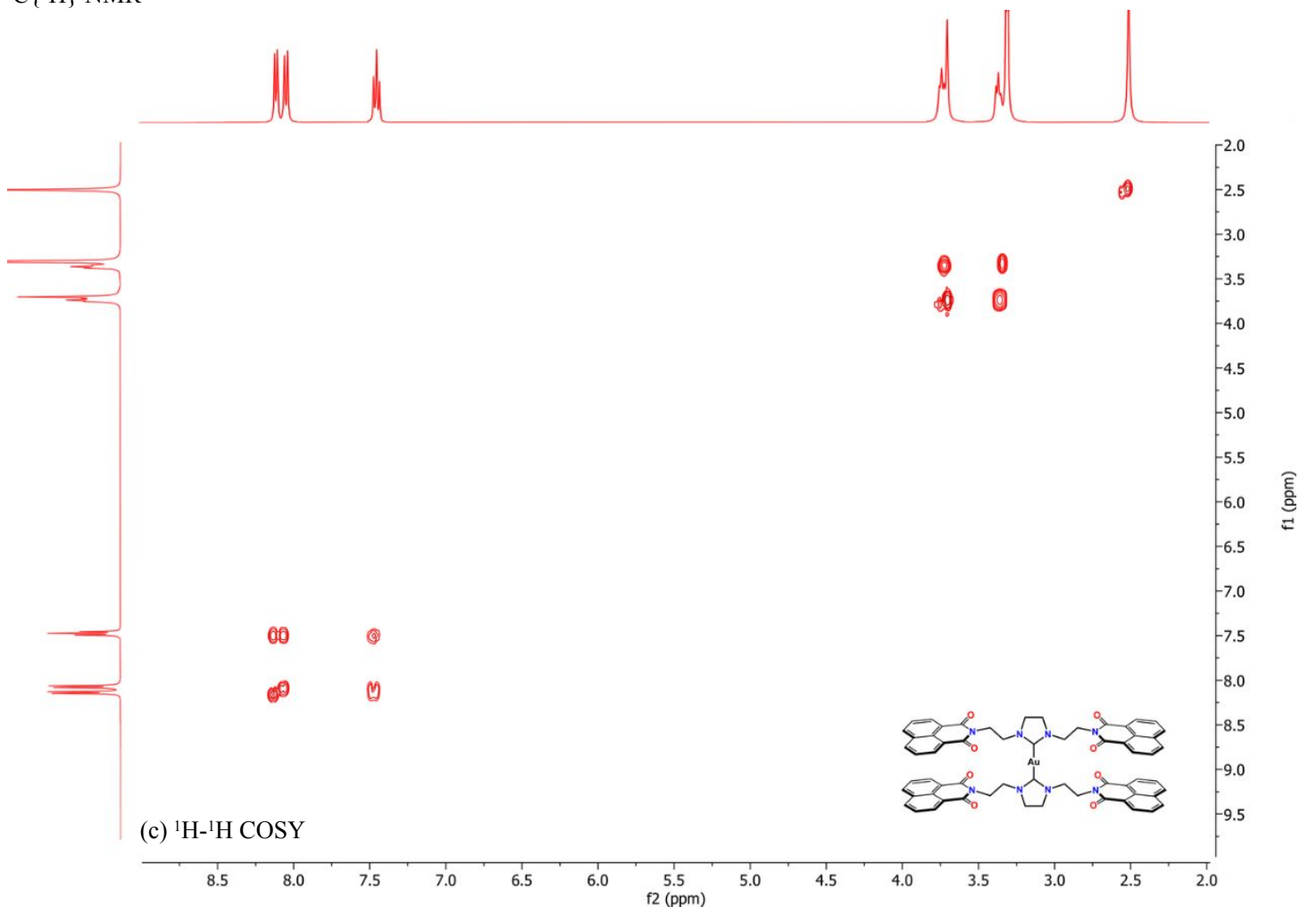

Figure S11. (a) ${ }^{1} \mathrm{H}$ NMR spectrum of 4 in DMSO- $d_{6}$ at $298 \mathrm{~K}$. (b) ${ }^{13} \mathrm{C}\left\{{ }^{1} \mathrm{H}\right\}$ NMR spectrum of 4 in DMSO- $d_{6}$ at $298 \mathrm{~K}$. (c) ${ }^{1} \mathrm{H}-{ }^{1} \mathrm{H}$ COSY NMR spectrum of $\mathbf{4}$ in DMSO- $d_{6}$ at $298 \mathrm{~K}$. 

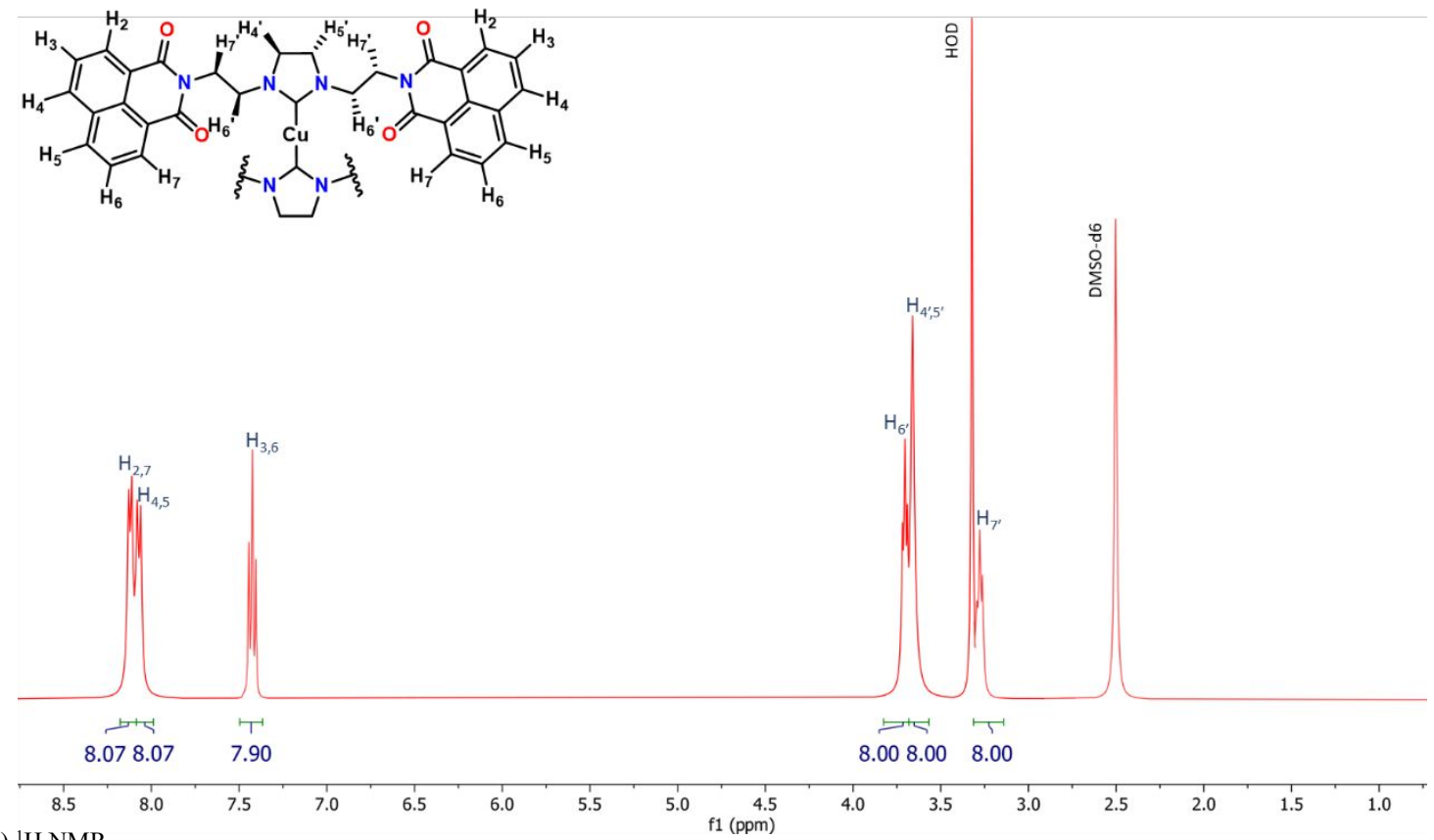

(a) ${ }^{1} \mathrm{H}$ NMR
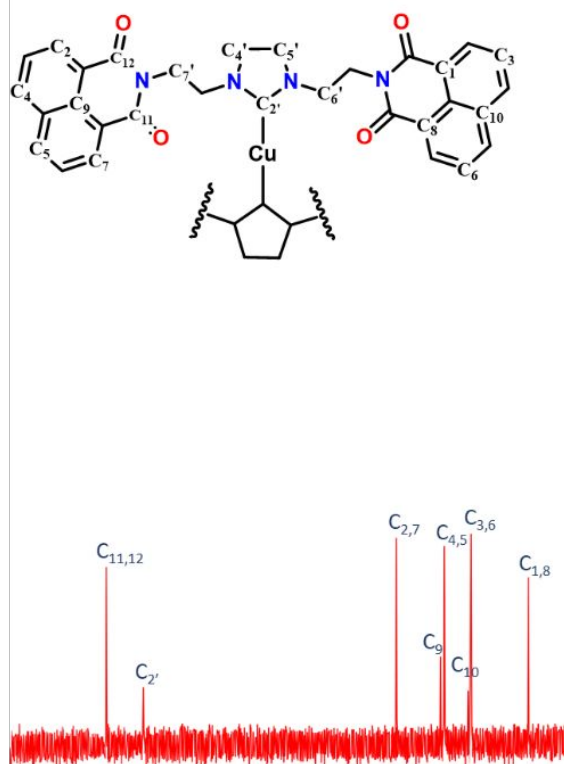

(b) ${ }^{13} \mathrm{C}\left\{{ }^{1} \mathrm{H}\right\} \mathrm{NMR}$ 


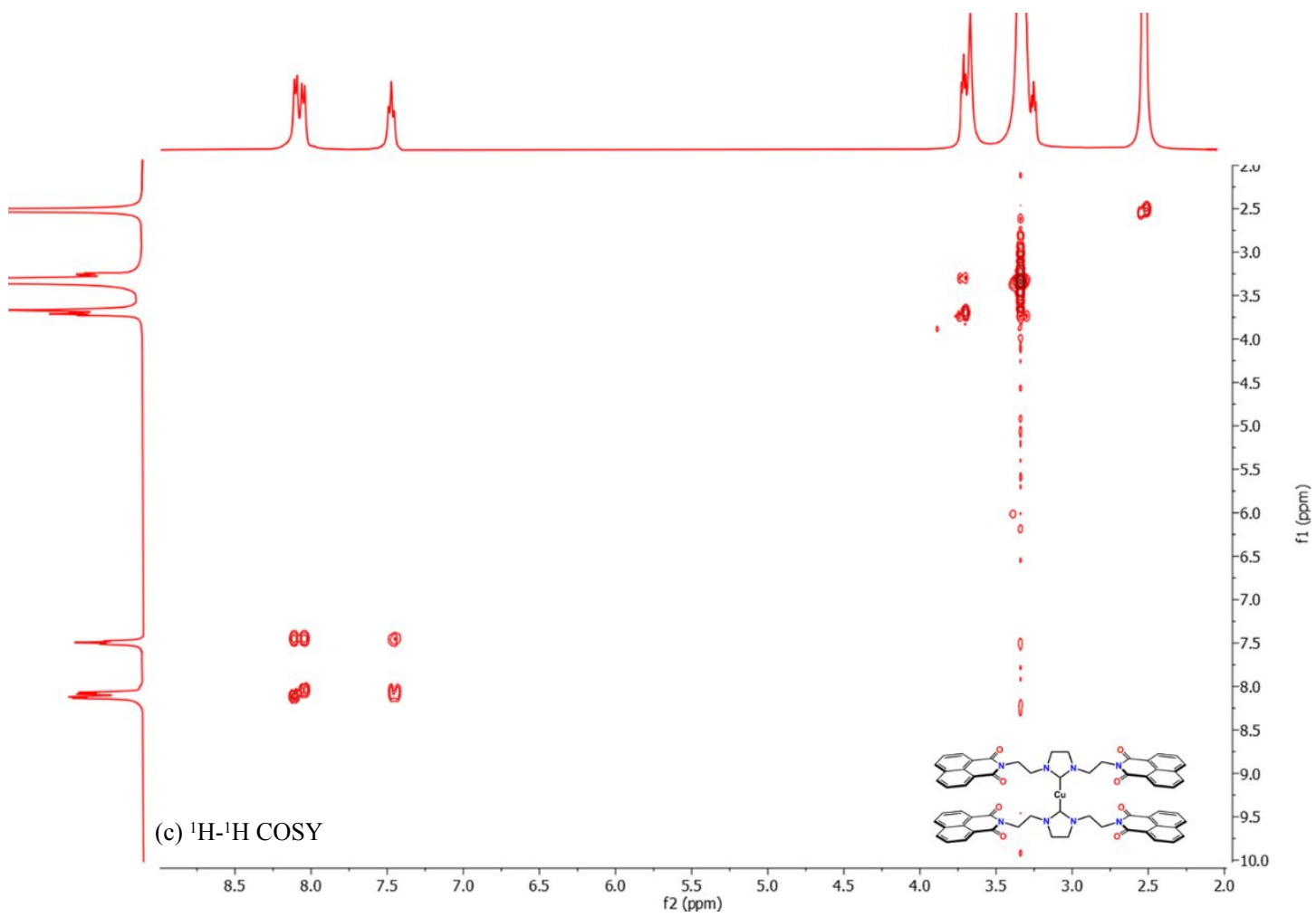

Figure S12. (a) ${ }^{1} \mathrm{H}$ NMR spectrum of 5 in DMSO- $d_{6}$ at $298 \mathrm{~K}$. (b) ${ }^{13} \mathrm{C}\left\{{ }^{1} \mathrm{H}\right\}$ NMR spectrum of 5 in DMSO- $d_{6}$ at $298 \mathrm{~K}$. (c) ${ }^{1} \mathrm{H}-{ }^{1} \mathrm{H}$ COSY NMR spectrum of 5 in DMSO- $d_{6}$ at $298 \mathrm{~K}$.
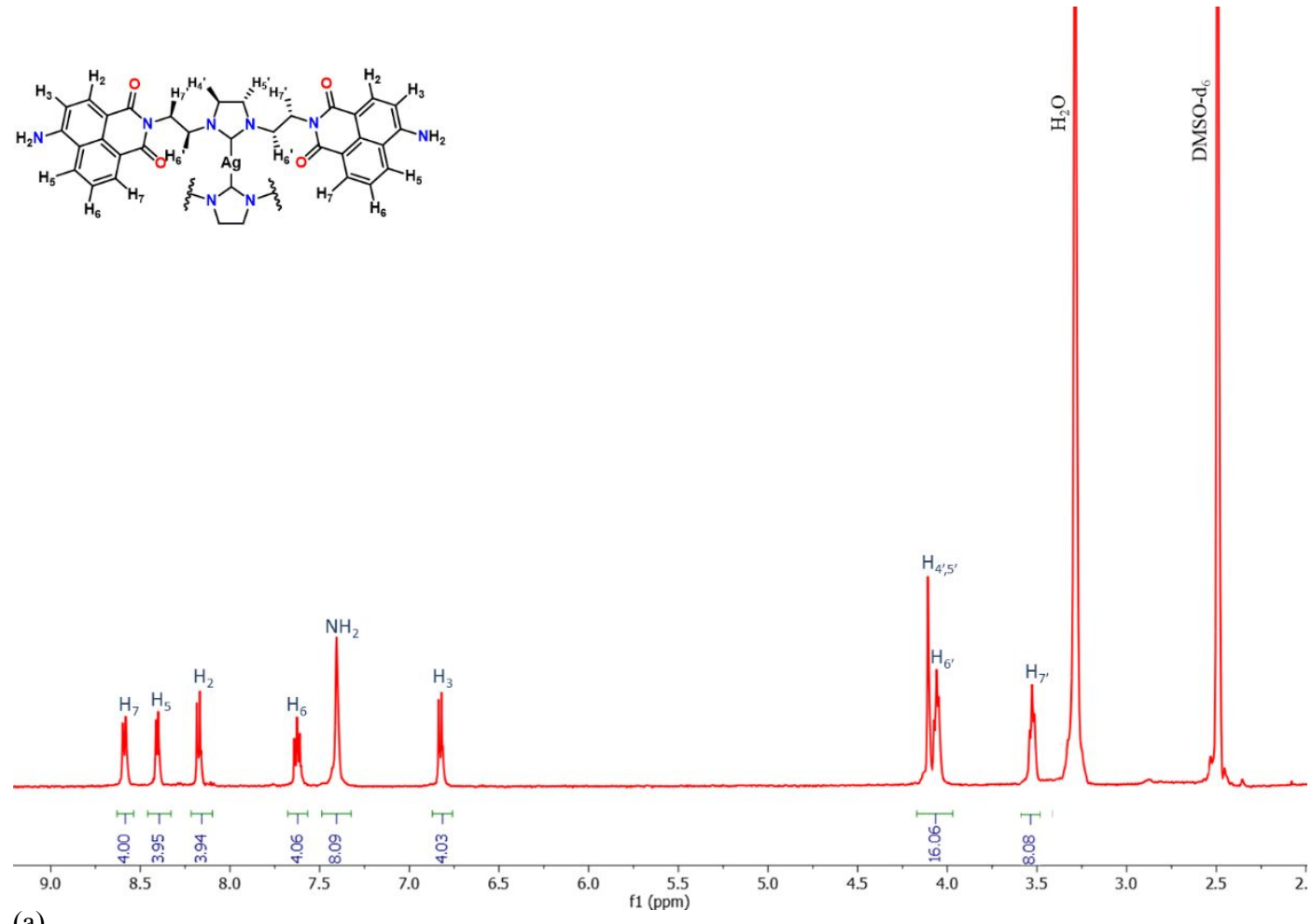

(a) 

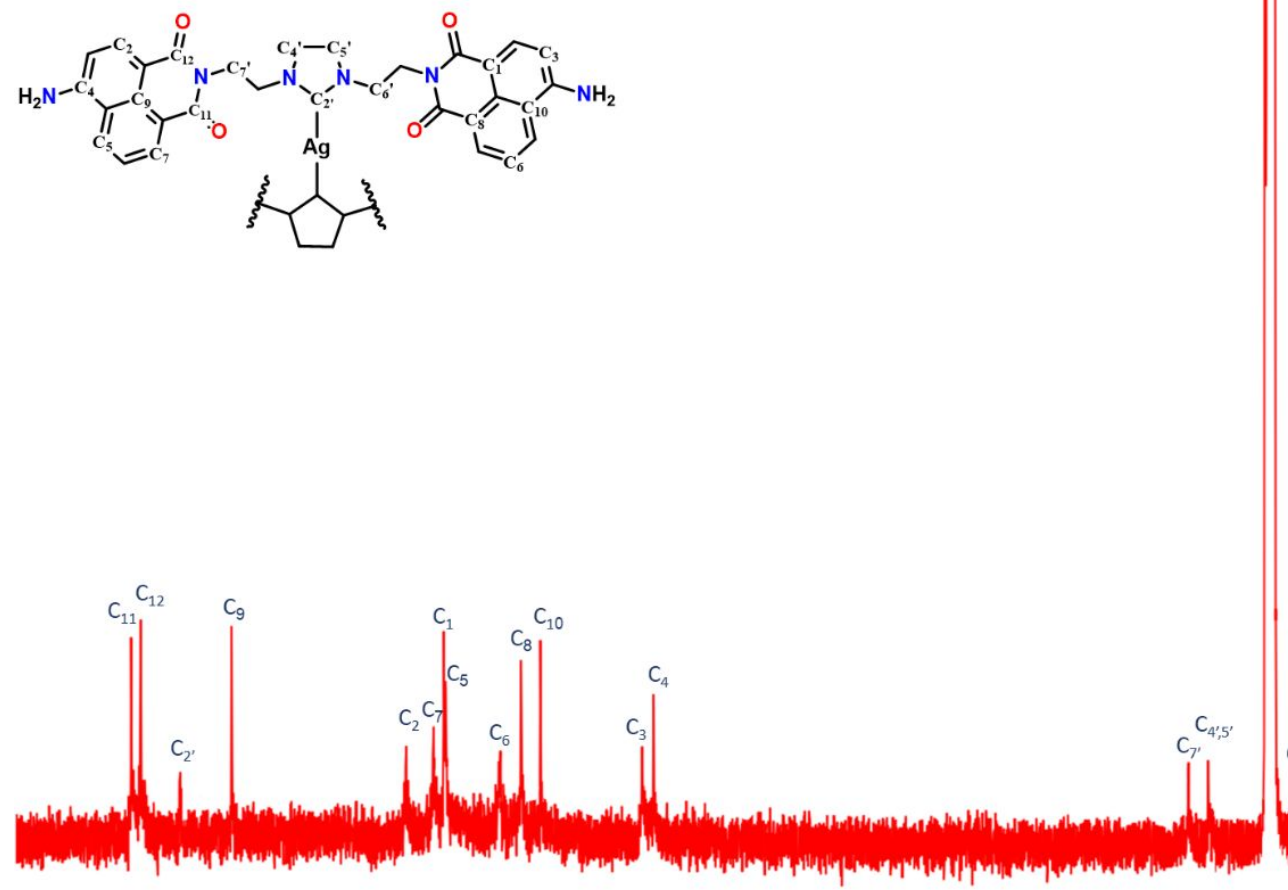

(b)
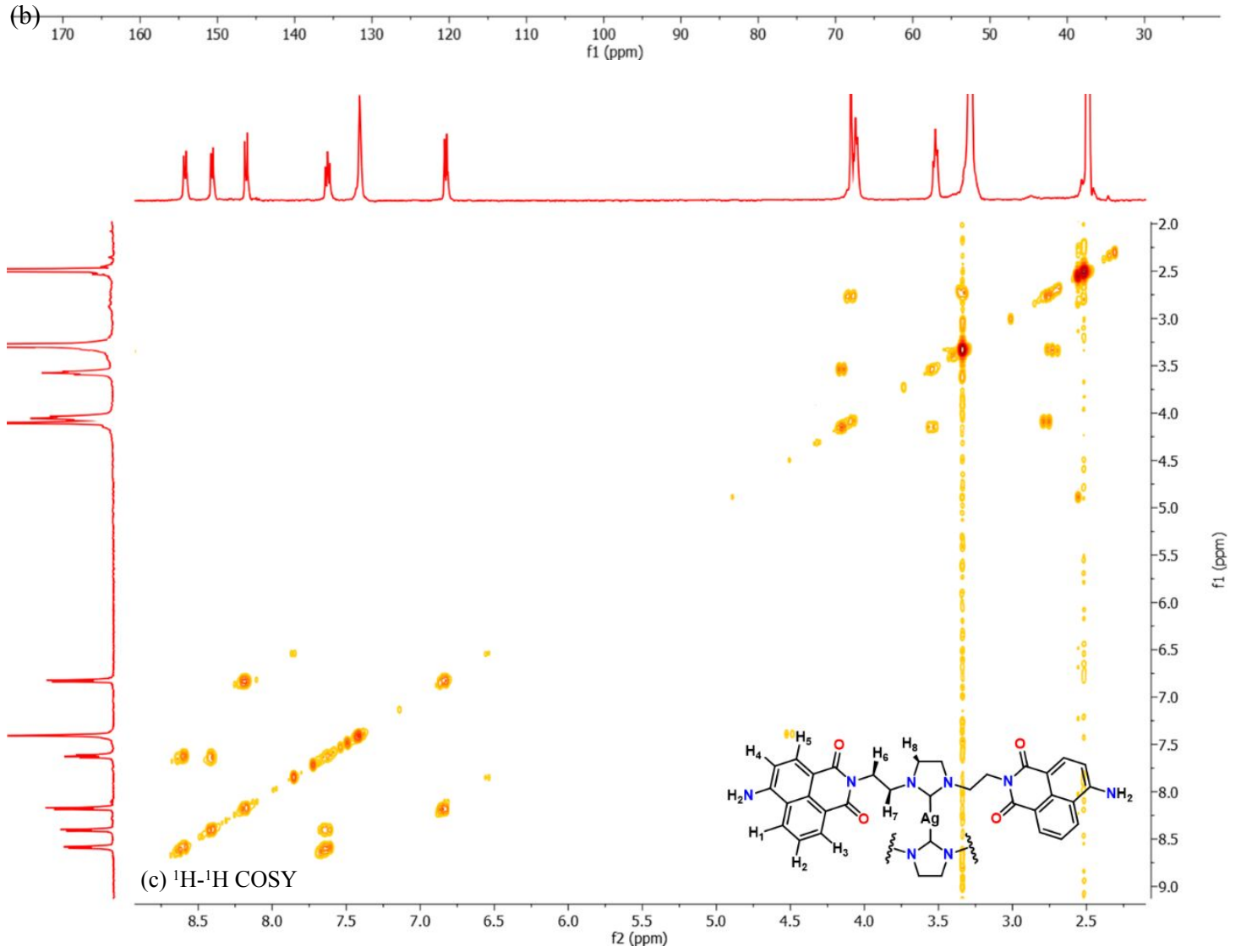

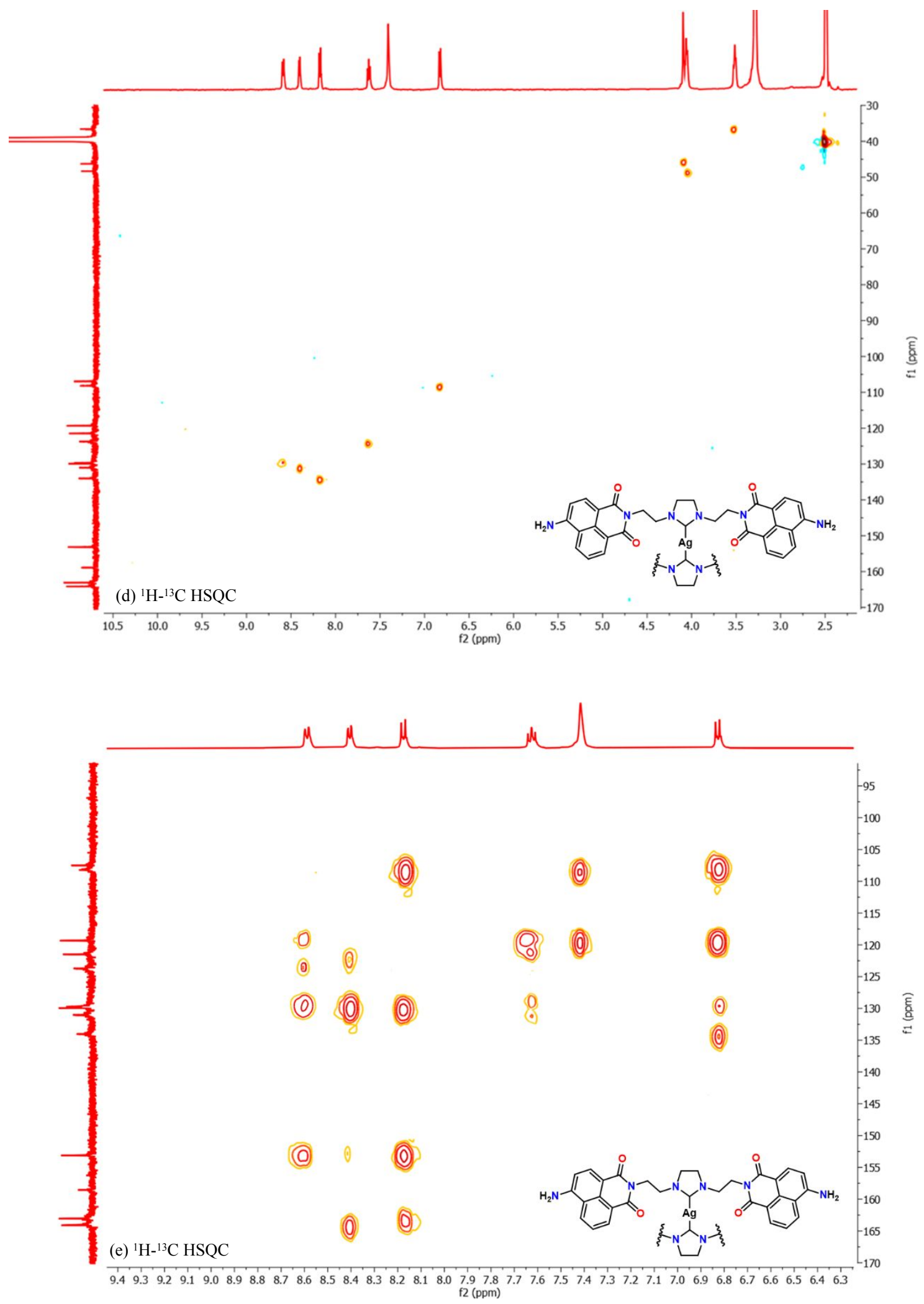

Figure S13. (a) ${ }^{1} \mathrm{H}$ NMR spectrum of 6 in DMSO- $d_{6}$ at $298 \mathrm{~K}$. (b) ${ }^{13} \mathrm{C}\left\{{ }^{1} \mathrm{H}\right\}$ NMR spectrum of 6 in DMSO- $d_{6}$ at $298 \mathrm{~K}$. (c) ${ }^{1} \mathrm{H}-{ }^{1} \mathrm{H}$ COSY NMR spectrum of 6 in DMSO- $d_{6}$ at $298 \mathrm{~K}$. (d) ${ }^{1} \mathrm{H}-{ }^{13} \mathrm{C}$ HSQC NMR spectrum of 6 in DMSO- $d_{6}$ at $298 \mathrm{~K}$. (e) ${ }^{1} \mathrm{H}-{ }^{13} \mathrm{C}$ HMBC NMR spectrum of $\mathbf{6}$, aromatic part, in DMSO- $d_{6}$ at $298 \mathrm{~K}$. 


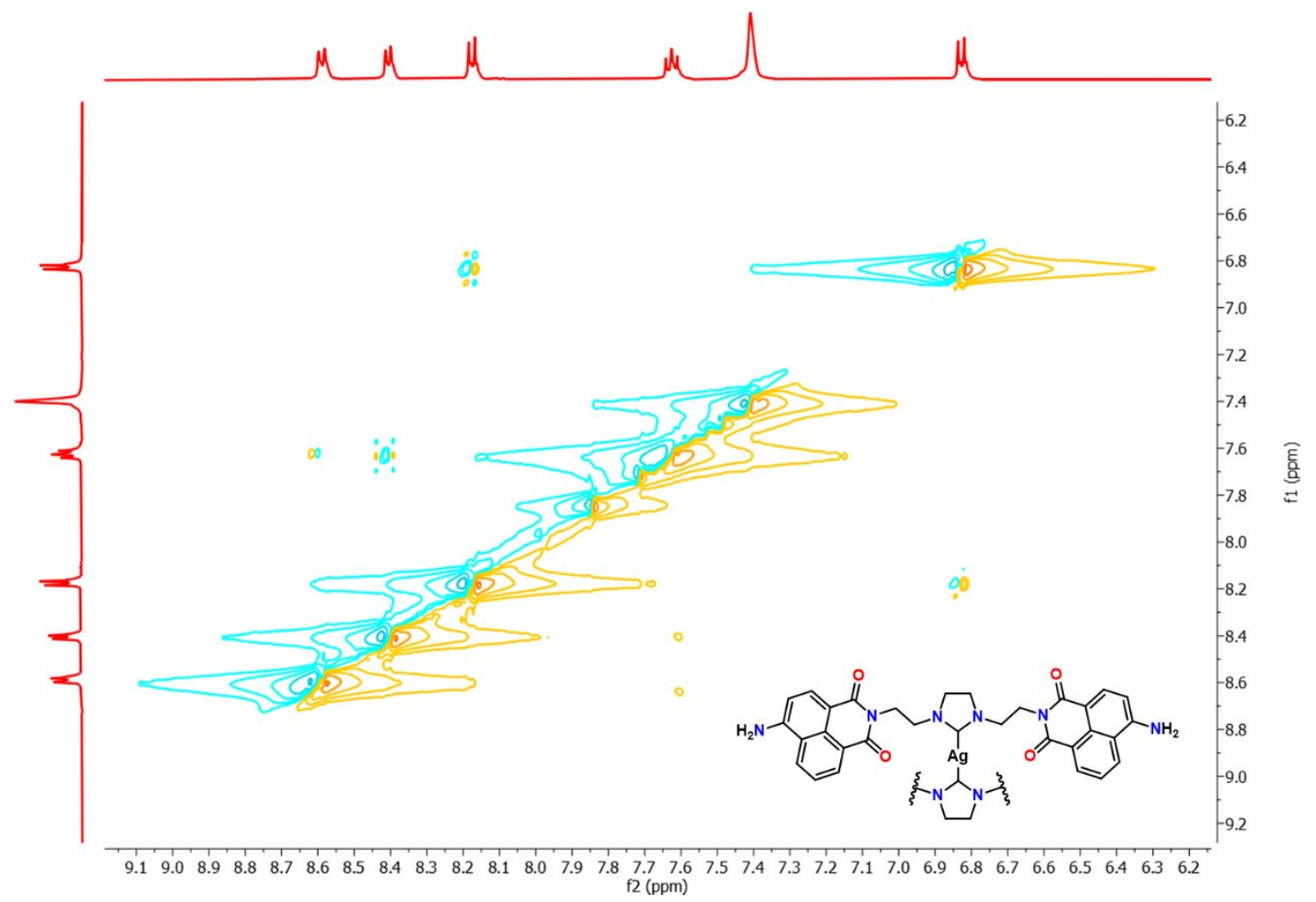

Figure S14. ${ }^{1} \mathrm{H}-{ }^{1} \mathrm{H}$ NOSY NMR of 6 in DMSO- $\mathrm{d}_{6}$ at $298 \mathrm{~K}$.

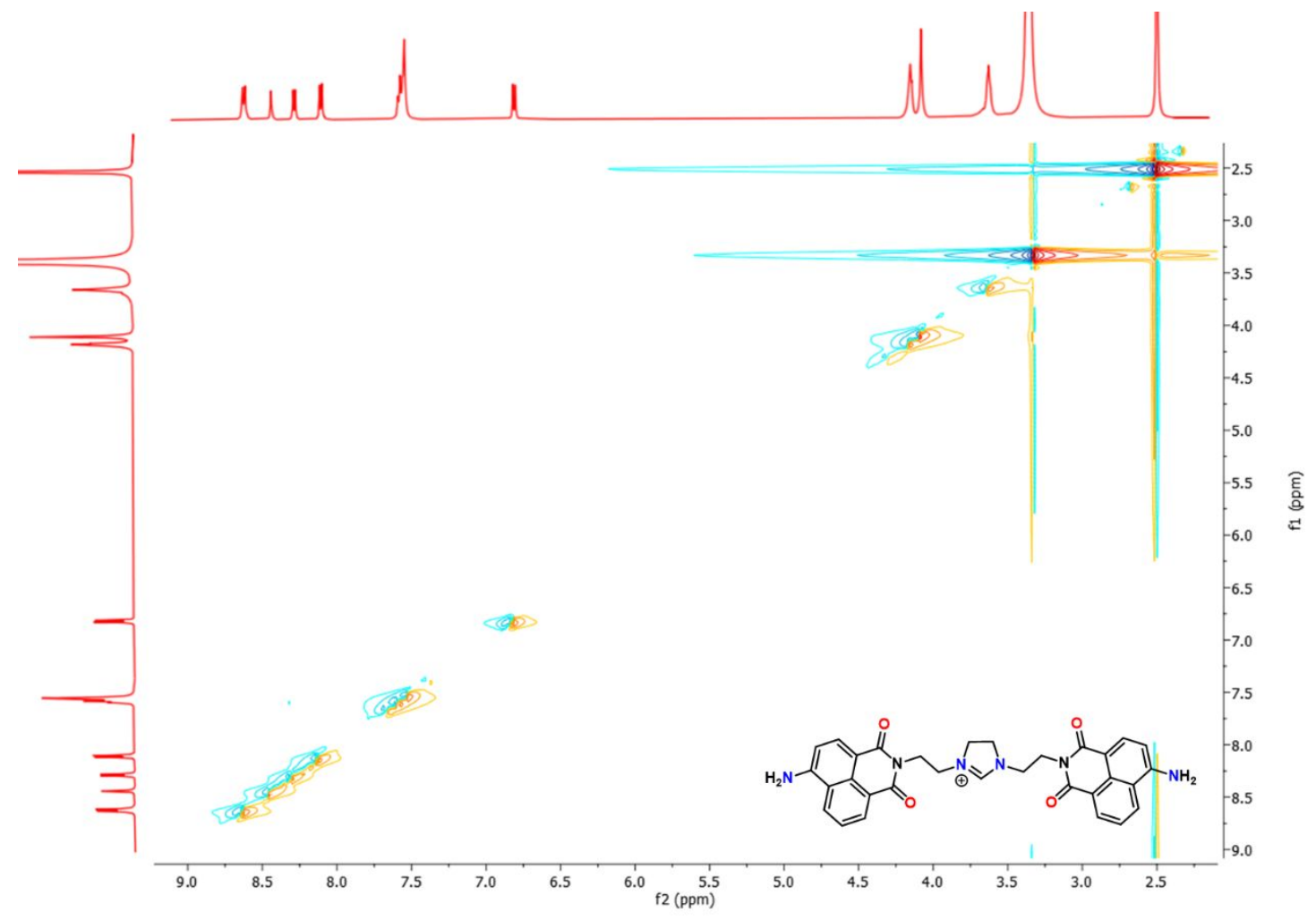

Figure S15. ${ }^{1} \mathrm{H}-{ }^{1} \mathrm{H}$ NOSY NMR spectrum of $2 \mathbf{c}$ in DMSO- $d_{6}$ at $298 \mathrm{~K}$. 


\section{$\mathrm{CD}_{3} \mathrm{CN}$}

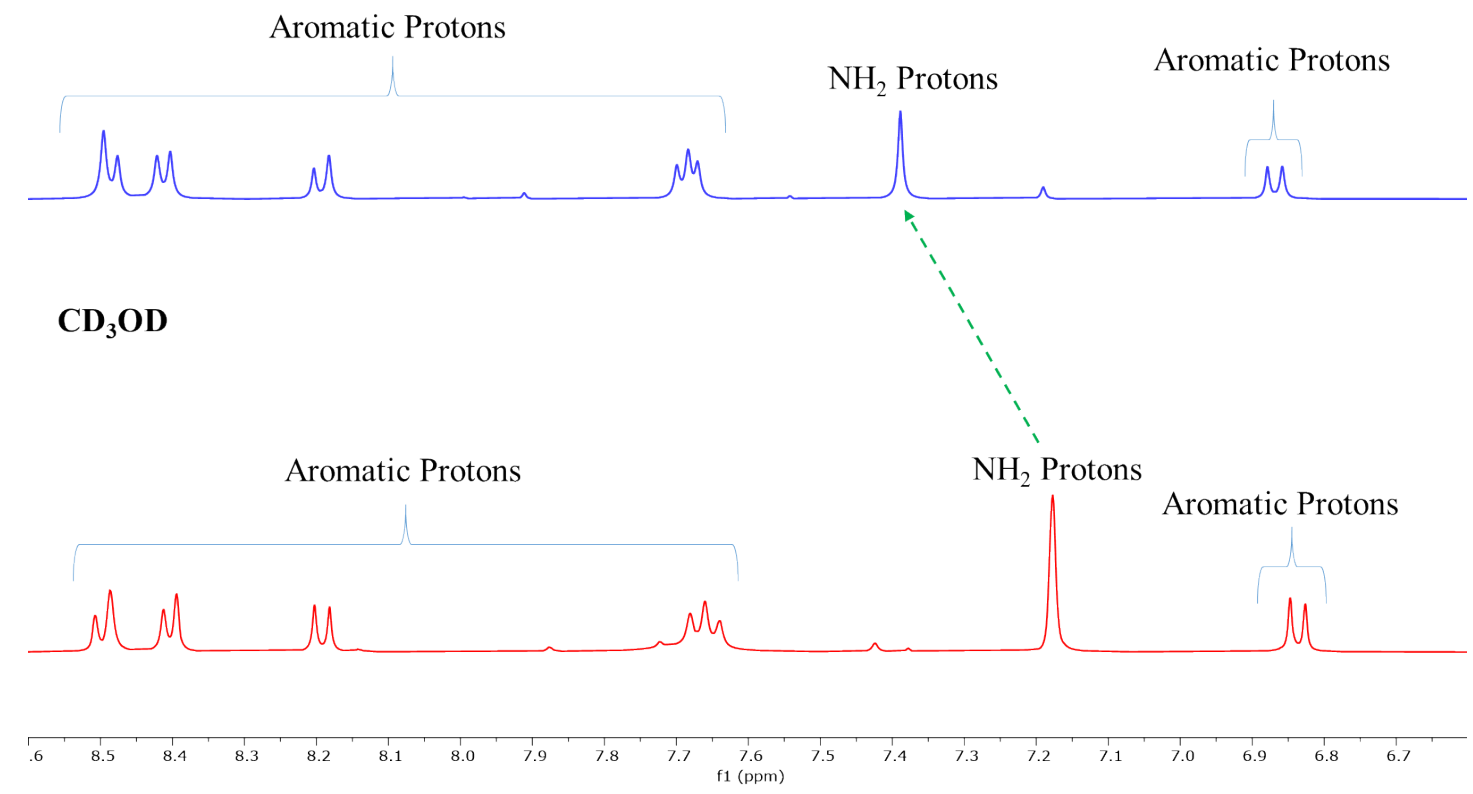

Figure S16. ${ }^{1} \mathrm{H}-\mathrm{NMR}$ spectrum of 6 in $\mathrm{CD}_{3} \mathrm{OD}-d_{4}$ and $\mathrm{CD}_{3} \mathrm{CN}-d_{3}$ at $298 \mathrm{~K}$.

6

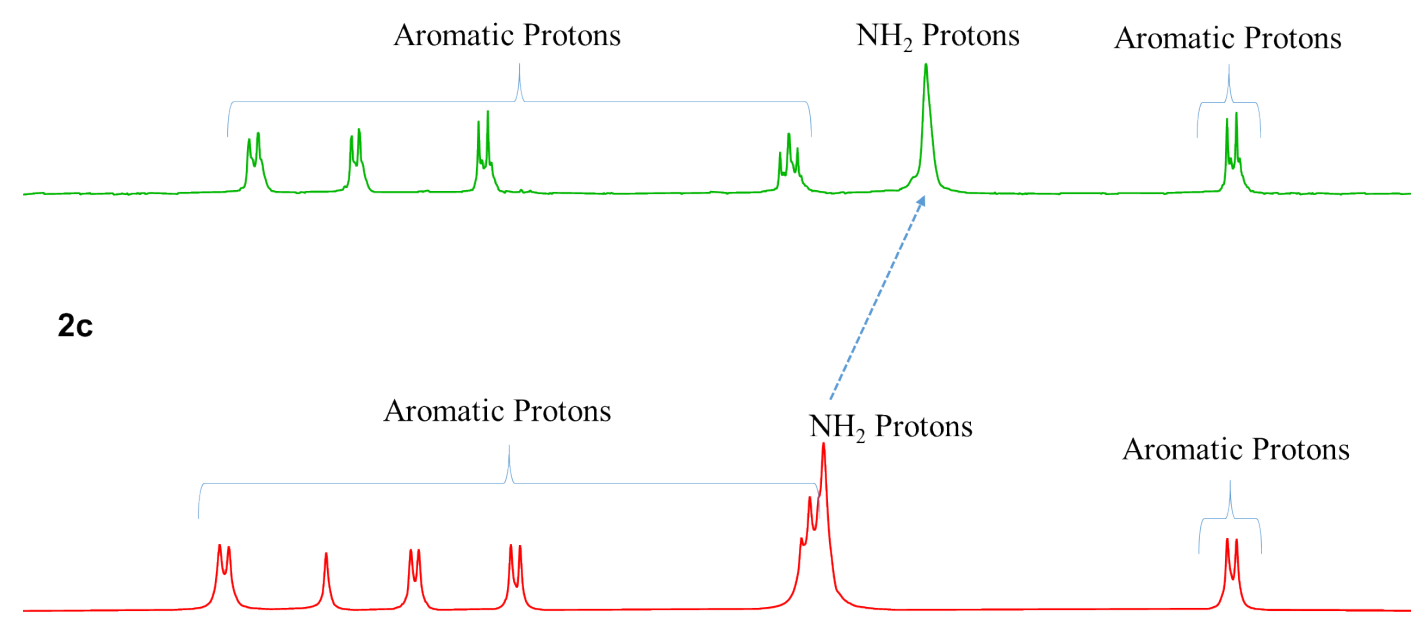

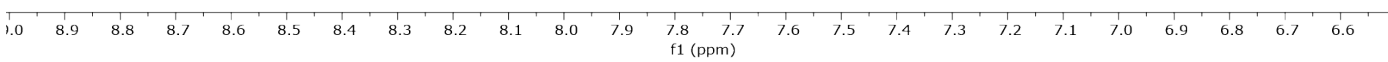

Figure S17. ${ }^{1} \mathrm{H}-\mathrm{NMR}$ spectrum of $\mathbf{2 c}$ and 6 in DMSO- $d_{6}$ at $298 \mathrm{~K}$. 


\section{ESI-Mass}

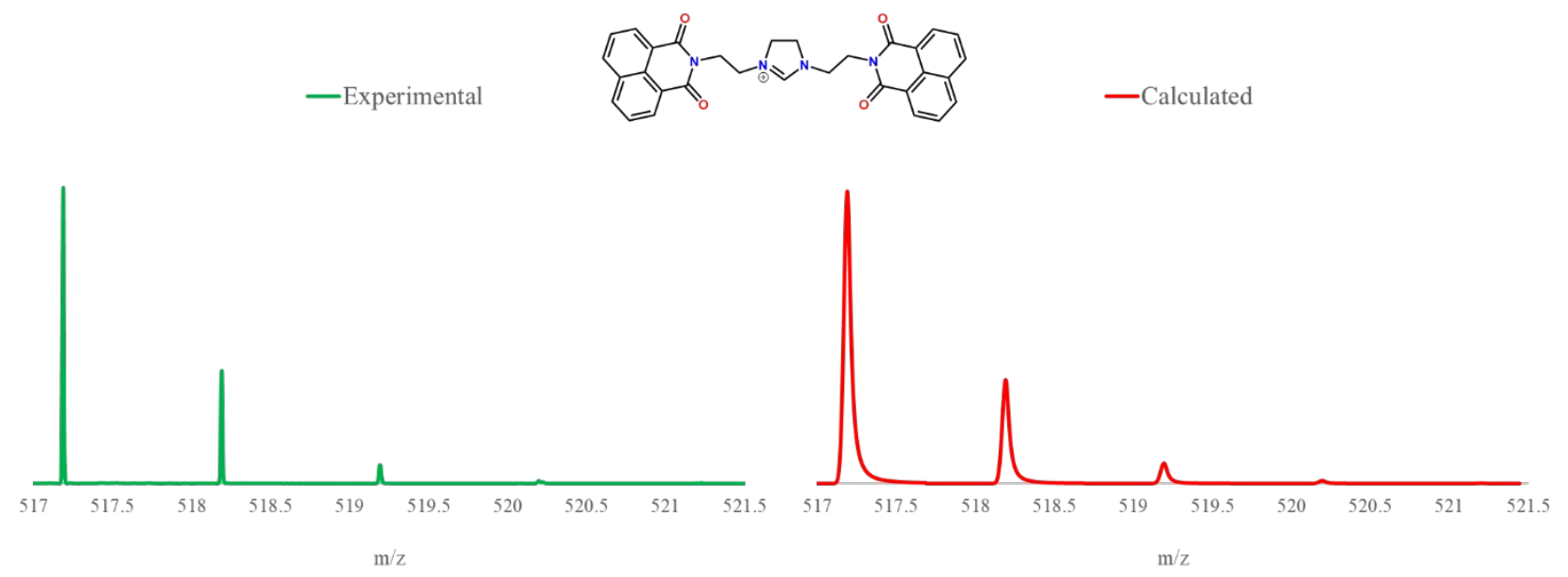

Figure S18. ESI ${ }^{+} \mathrm{HR}-\mathrm{MS}$ spectrum of 2a.(green) Expansion of the cationic fragment signal $\left[\mathrm{C}_{31} \mathrm{H}_{25} \mathrm{~N}_{4} \mathrm{O}_{4}\right]^{+}$at $\mathrm{m} / \mathrm{z}=517$, (red) simulated osotopic pattern of the cationic fragment $\left[\mathrm{C}_{31} \mathrm{H}_{25} \mathrm{~N}_{4} \mathrm{O}_{4}\right]^{+}$

- Experimental

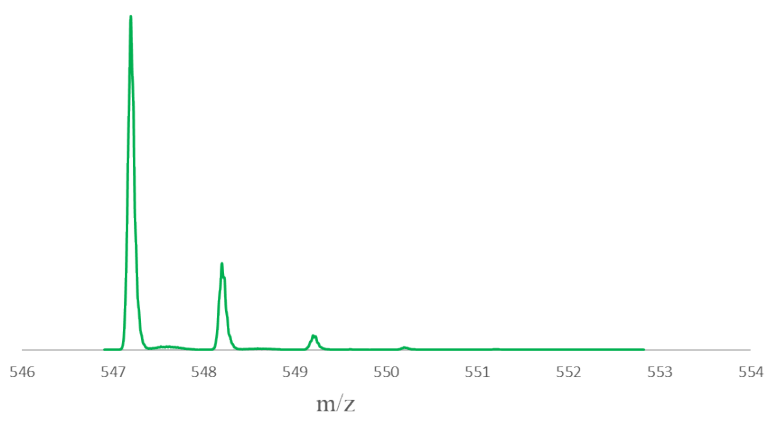

- Calculated

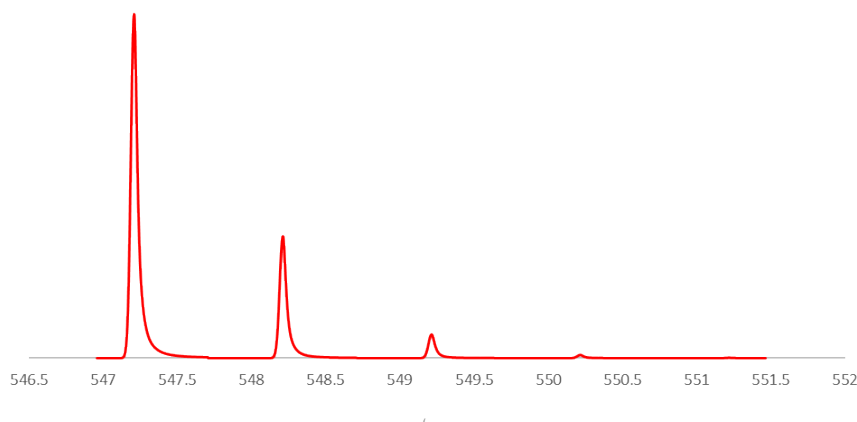

$\mathrm{m} / \mathrm{z}$

Figure S19. ESI ${ }^{+} \mathrm{HR}-\mathrm{MS}$ spectrum of 2c. (green) Expansion of the cationic fragment signal $\left[\mathrm{C}_{31} \mathrm{H}_{27} \mathrm{~N}_{6} \mathrm{O}_{4}\right]^{+}$at $\mathrm{m} / \mathrm{z}=547$, (red) simulated isotopic pattern of the cationic fragment $\left[\mathrm{C}_{31} \mathrm{H}_{27} \mathrm{~N}_{6} \mathrm{O}_{4}\right]^{+}$

-Experimental

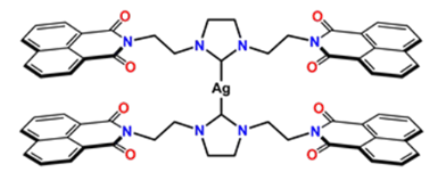

- Calculated

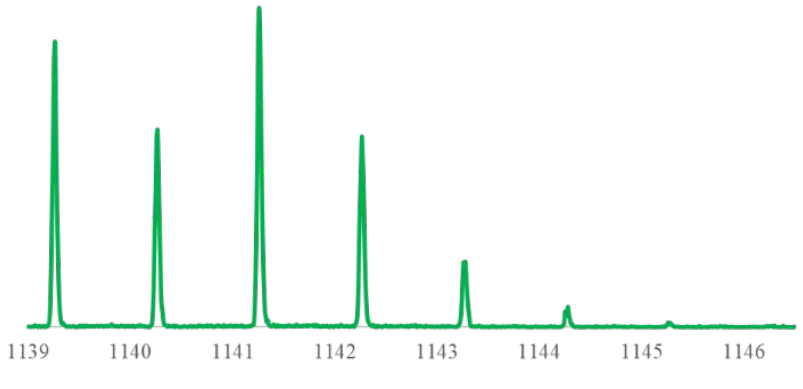

$\mathrm{m} / \mathrm{z}$

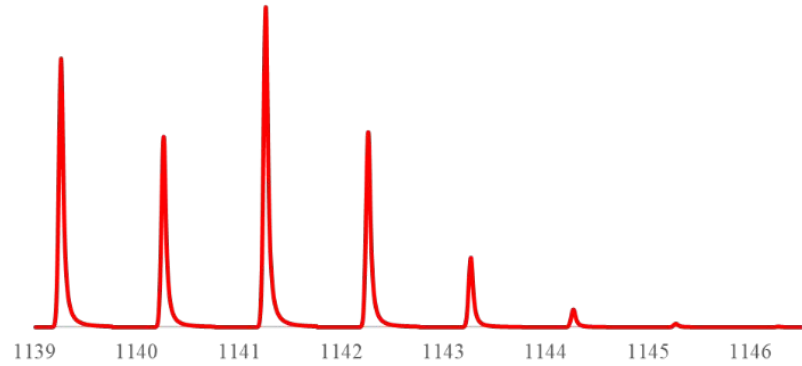

$\mathrm{m} / \mathrm{z}$

Figure S20. ESI $^{+}$HRMS spectrum of 3. (green) Expansion of the dicationic fragment signal $\left[\mathrm{C}_{62} \mathrm{H}_{48} \mathrm{AgN}_{8} \mathrm{O}_{8}\right]^{+}$at $\mathrm{m} / \mathrm{z}=1141$, (red) simulated isotopic pattern of the cationic fragment $\left[\mathrm{C}_{62} \mathrm{H}_{48} \mathrm{AgN}_{8} \mathrm{O}_{8}\right]^{+}$ 


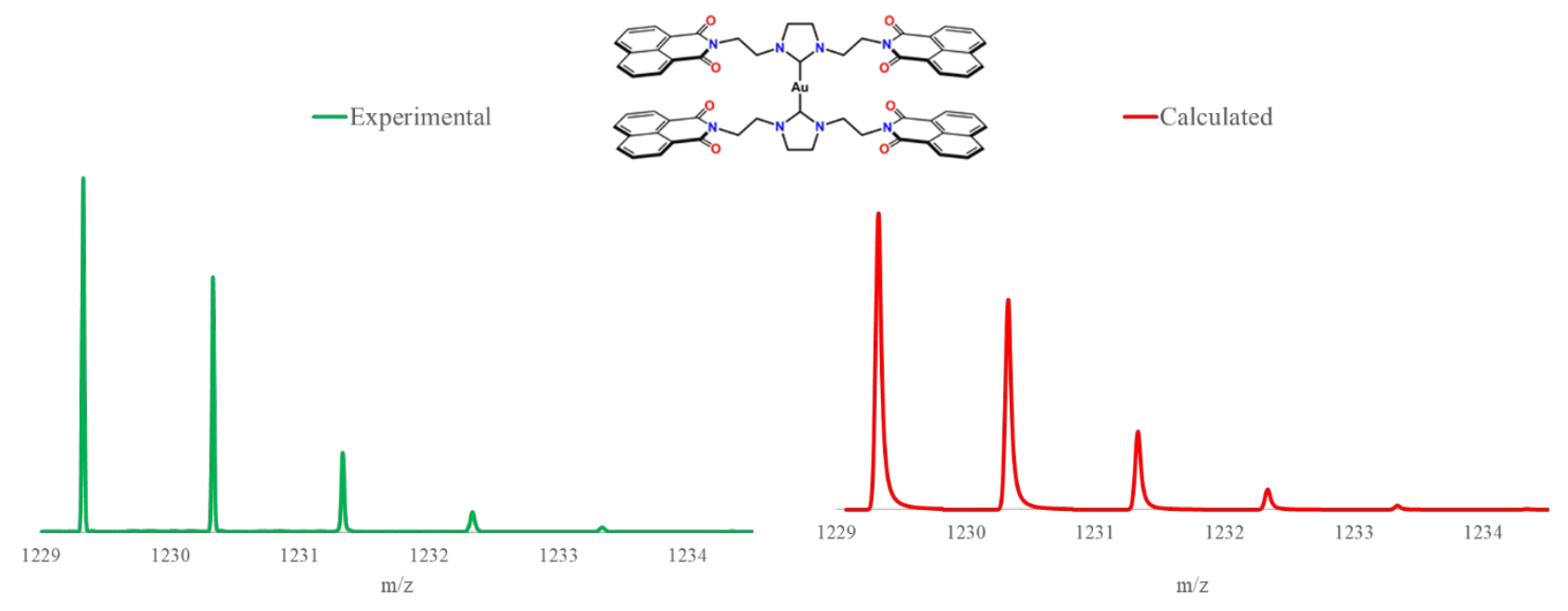

Figure S21. $\mathrm{ESI}^{+} \mathrm{HRMS}$ spectrum of 4. (green) Expansion of the cationic fragment signal $\left[\mathrm{C}_{62} \mathrm{H}_{48} \mathrm{AuN}_{8} \mathrm{O}_{8}\right]^{+}$at $\mathrm{m} / \mathrm{z}=1229$, (red) simulated isotopic pattern of the cationic fragment $\left[\mathrm{C}_{62} \mathrm{H}_{48} \mathrm{AuN}_{8} \mathrm{O}_{8}\right]^{+}$

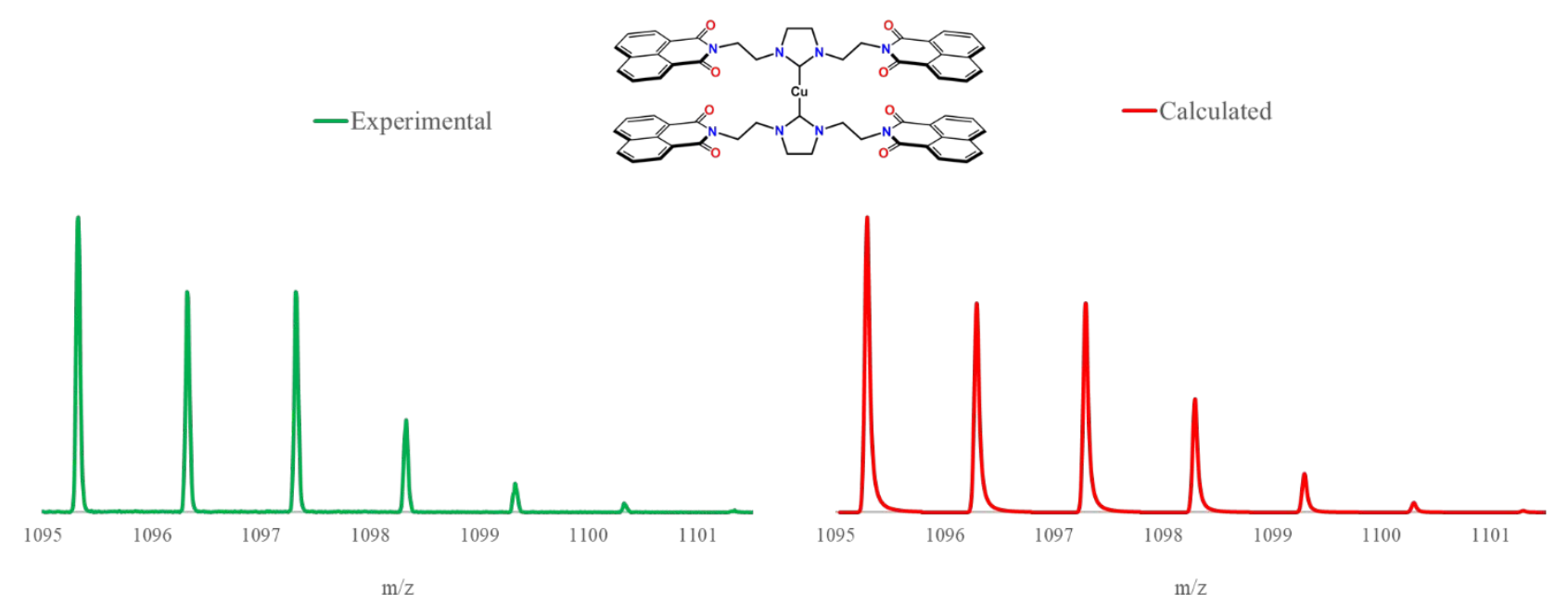

Figure S22. ESI ${ }^{+}$HRMS spectrum of 5. (green) Expansion of the cationic fragment signal $\left[\mathrm{C}_{62} \mathrm{H}_{48} \mathrm{AuN}_{8} \mathrm{O}_{8}\right]^{+}$at $\mathrm{m} / \mathrm{z}=1095$, (red) simulated isotopic pattern of the cationic fragment $\left[\mathrm{C}_{62} \mathrm{H}_{48} \mathrm{AuN}_{8} \mathrm{O}_{8}\right]^{+}$

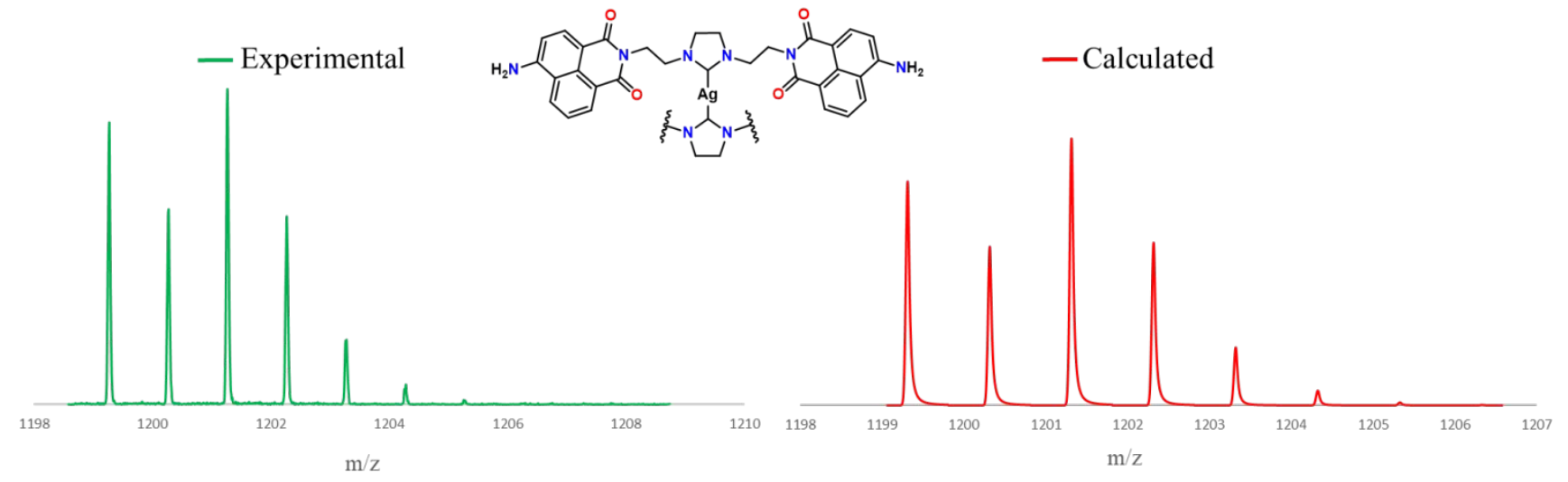

Figure S23. ESI $^{+}$HRMS spectrum of 6. (green) Expansion of the cationic fragment signal $\left[\mathrm{C}_{62} \mathrm{H}_{52} \mathrm{AgN}_{12} \mathrm{O}_{8}\right]^{+}$at $\mathrm{m} / \mathrm{z}=1201$, (red) simulated isotopic pattern of the cationic fragment $\left[\mathrm{C}_{62} \mathrm{H}_{52} \mathrm{AgN}_{12} \mathrm{O}_{8}\right]^{+}$ 


\section{Crystal Structures}

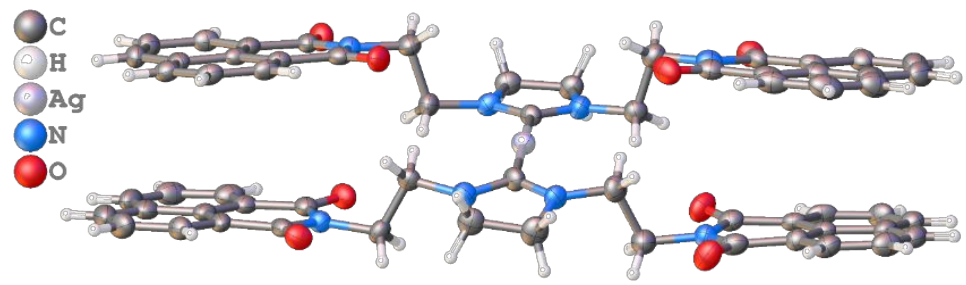

Figure S24. Molecular crystal structure of $\mathbf{3}$. The anions $\left(\mathrm{PF}_{6}\right)$ and solvents of crystallization have been omitted for the sake of clarity.

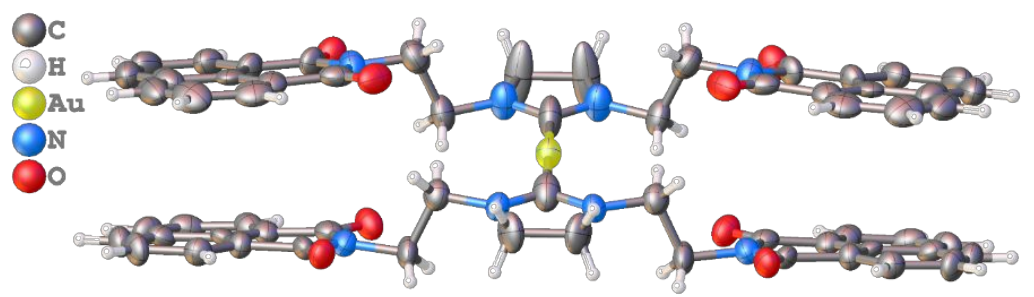

Figure S25. Molecular crystal structure of 4 . The anions $\left(\mathrm{PF}_{6}\right)$ and solvents of crystallization have been omitted for the sake of clarity.

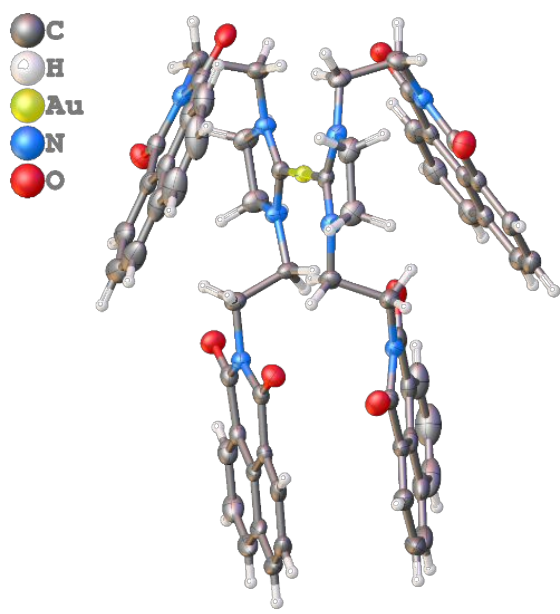

Figure S26. Molecular crystal structure of 4 . The anions $\left(\mathrm{PF}_{6}\right)$ and solvents of crystallization have been omitted for the sake of clarity.

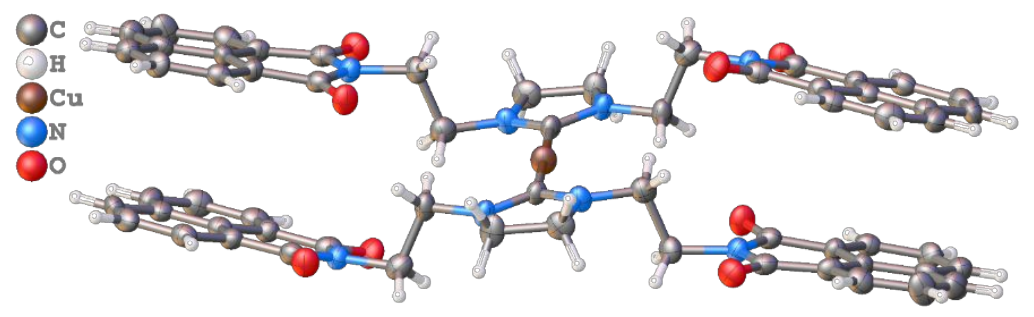

Figure S27. Molecular crystal structure of 5 . The anions $\left(\mathrm{PF}_{6}\right)$ and solvents of crystallization have been omitted for the sake of clarity. 
Table S1. Crystal data and structure refinement for 3-5 complexes.

\begin{tabular}{|c|c|c|c|c|}
\hline \multirow[t]{2}{*}{ Parameter } & \multicolumn{3}{|c|}{ Title } & \multirow[b]{2}{*}{5} \\
\hline & 3 & 4 (Parallel) & 4 (Twisted) & \\
\hline Formula & $\mathrm{C}_{65} \mathrm{H}_{54} \mathrm{AgCl}_{6} \mathrm{~F}_{6} \mathrm{~N}_{8} \mathrm{O}_{8} \mathrm{P}$ & $\mathrm{C}_{64} \mathrm{H}_{50} \mathrm{AuCl}_{6} \mathrm{~F}_{6} \mathrm{~N}_{8} \mathrm{O}_{8} \mathrm{P}$ & $\mathrm{C}_{63} \mathrm{H}_{50} \mathrm{AuCl}_{2} \mathrm{~F}_{6} \mathrm{~N}_{8} \mathrm{O}_{8} \mathrm{P}$ & $\mathrm{C}_{64} \mathrm{H}_{52} \mathrm{Cl}_{4} \mathrm{CuF}_{6} \mathrm{~N}_{8} \mathrm{O}_{8} \mathrm{P}$ \\
\hline Molecular weight & 1540.7 & 1613.75 & 1459.94 & 1411.44 \\
\hline Temperature (K) & 296.15 & 296.15 & 296.15 & 296.15 \\
\hline Wavelength & $1.54178 \AA$ & $1.54178 \AA$ & $0.71073 \AA$ & $0.71073 \AA$ \\
\hline Crystal System & Monoclinic & Orthorhombic & Monoclinic & Triclinic \\
\hline Space group & $\mathrm{P} 2_{1} / \mathrm{c}$ & Pnma & $\mathrm{P} 2_{1} / \mathrm{c}$ & P-1 \\
\hline$a(\AA)$ & $16.5813(10)$ & $15.3494(6)$ & $11.3974(13)$ & $10.059(12)$ \\
\hline$b(\AA)$ & $26.6233(15)$ & $26.8313(11)$ & $24.938(3)$ & $11.880(14)$ \\
\hline$c(\AA)$ & $15.4322(9)$ & $14.9046(6)$ & $19.928(2)$ & $13.154(16)$ \\
\hline$\alpha\left({ }^{\circ}\right)$ & 90 & 90 & 90 & $76.159(15)$ \\
\hline$\beta\left(^{\circ}\right)$ & $111.891(3)$ & 90 & $94.7470(10)$ & $78.177(15)$ \\
\hline$\gamma\left({ }^{\circ}\right)$ & 90 & 90 & 90 & $81.574(15)$ \\
\hline$V\left(\AA^{3}\right)$ & $6321.3(7)$ & $6138.4(4)$ & $5644.7(11)$ & 1486(3) \\
\hline$Z$ & 4 & 4 & 4 & 1 \\
\hline$D_{\text {calc }}\left(\mathrm{g} / \mathrm{cm}^{3}\right)$ & 1.619 & 1.746 & 1.718 & 1.577 \\
\hline$\mu\left(\mathrm{mm}^{-1}\right)$ & 5.834 & 7.872 & 2.817 & 0.659 \\
\hline \multirow[t]{2}{*}{$\mathrm{F}(000)$} & 3128 & 3216 & 2920 & 722 \\
\hline & $-18 \leq h \leq 13$ & $-14 \leq h \leq 16$ & $-14 \leq h \leq 14$ & $-12 \leq h \leq 12$ \\
\hline \multirow[t]{2}{*}{ Index ranges } & $-27 \leq k \leq 29$ & $-26 \leq k \leq 29$ & $-32 \leq k \leq 32$ & $-14 \leq k \leq 14$ \\
\hline & $-16 \leq l \leq 16$ & $-13 \leq l \leq 16$ & $-25 \leq l \leq 25$ & $-15 \leq l \leq 15$ \\
\hline $\begin{array}{l}\text { Data / restraints / } \\
\text { parameters }\end{array}$ & $7311 / 20 / 856$ & $4222 / 0 / 439$ & $12529 / 0 / 802$ & $5413 / 0 / 419$ \\
\hline Crystal size $\left(\mathrm{mm}^{3}\right)$ & $0.5 \times 0.2 \times 0.2$ & $0.6 \times 0.2 \times 0.1$ & $0.7 \times 0.2 \times 0.2$ & $0.8 \times 0.2 \times 0.1$ \\
\hline Angle range $2 \theta\left(^{\circ}\right)$ & 5.744 to $116.314^{\circ}$ & 4.304 to $54.392^{\circ}$ & 6.588 to $116.65^{\circ}$ & 4.16 to $50.96^{\circ}$ \\
\hline $\begin{array}{l}\text { Reflection } \\
\text { Collected }\end{array}$ & 17481 & 63985 & 19101 & 14358 \\
\hline $\begin{array}{l}\text { Independent } \\
\text { Reflection }\end{array}$ & 7311 & 4222 & 12529 & 5413 \\
\hline$R_{\text {int }}$ & 0.0278 & 0.0472 & 0.0305 & 0.1135 \\
\hline$R_{\sigma}$ & 0.0389 & 0.0561 & 0.0219 & 0.1519 \\
\hline $\begin{array}{l}\text { Absorption } \\
\text { correction }\end{array}$ & Multi Scan & Multi Scan & Multi Scan & Multi Scan \\
\hline $\begin{array}{l}\text { Refinement } \\
\text { method }\end{array}$ & $\begin{array}{l}\text { Full-matrix least-squares } \\
\text { on } \mathrm{F}^{2}\end{array}$ & $\begin{array}{l}\text { Full-matrix least- } \\
\text { squares on } \mathrm{F}^{2}\end{array}$ & $\begin{array}{l}\text { Full-matrix least- } \\
\text { squares on } \mathrm{F}^{2}\end{array}$ & $\begin{array}{l}\text { Full-matrix least- } \\
\text { squares on } \mathrm{F}^{2}\end{array}$ \\
\hline $\begin{array}{c}\text { Completeness to } \\
\text { theta }=25.242^{\circ}\end{array}$ & $83 \%$ & $95 \%$ & $100 \%$ & $100 \%$ \\
\hline$R_{1}\left(\left|F_{\mathrm{o}}\right| \geq 4 \sigma_{F}\right)$ & 0.0704 & 0.0721 & 0.0284 & 0.0677 \\
\hline$w R_{2}\left(\left|F_{\mathrm{o}}\right| \geq 4 \sigma_{F}\right)$ & 0.1982 & 0.1985 & 0.0706 & 0.1613 \\
\hline$R_{1}$ (all data) & 0.0808 & 0.0935 & 0.0327 & 0.1453 \\
\hline$w R_{2}$ (all data) & 0.2099 & 0.2195 & 0.0729 & 0.2019 \\
\hline GOOF & 1.055 & 1.138 & 1.044 & 0.892 \\
\hline$\rho_{\mathrm{uvv}}, \rho_{\mathrm{u \alpha} \tilde{k},}, \varepsilon / \oplus^{3}$ & $3.01 /-0.92$ & $1.24 /-2.43$ & $1.85 /-0.75$ & $0.74 /-0.76$ \\
\hline $\mathrm{CCDC}$ & 2070212 & 2070213 & 2070214 & 2070215 \\
\hline
\end{tabular}


Table S2. Selected interactions and bond lengths $[\AA]$ and angels $\left[{ }^{0}\right]$ of $\mathbf{3}$

\begin{tabular}{cccc}
\hline \multicolumn{2}{c}{ Bond Angles $\left[{ }^{0}\right]$} & \multicolumn{2}{c}{ Bond Length $(\AA)$} \\
\hline C1A-Ag-C1B & 174.28 & Ag-C1A & 2.09 \\
N1A-C1A-N1B & 107.89 & Ag-C1B & 2.077 \\
N00Q-C1B-N00M & 107.09 & C16A-C018 & 2.765 \\
NMI-NMI avg & 2.765 & CO (O1'A)-C011 & 3.399 \\
$\alpha$ & 33.01 & CO (O1'B)-C025 & 3.368 \\
\hline
\end{tabular}

Table S3. Selected interactions and bond lengths $[\AA]$ and angels $\left[{ }^{0}\right]$ of $\mathbf{4}$ (parallel)

\begin{tabular}{|c|c|c|c|}
\hline \multicolumn{2}{|c|}{ Bond Angles $\left[{ }^{0}\right]$} & \multicolumn{2}{|c|}{ Bond Length $(\AA)$} \\
\hline C1A-Au-C1B & 177.96 & $\mathrm{Au}-\mathrm{C} 1 \mathrm{~A}$ & 1.966 \\
\hline N1A-C1A-N1A & 105.4 & $\mathrm{Au}-\mathrm{C} 1 \mathrm{~B}$ & 1.978 \\
\hline N1B-C1B-N1B & 105.46 & C16A-C16B & 3.821 \\
\hline NMI-NMI avg & 2.23 & $\mathrm{CO}(\mathrm{O} 1 \mathrm{~A})-\mathrm{C} 15 \mathrm{~B}$ & 3.562 \\
\hline$\alpha$ & 34.72 & $\mathrm{CO}\left(\mathrm{O} 1^{\prime} \mathrm{B}\right)-\mathrm{C} 5 \mathrm{~A}$ & 3.751 \\
\hline
\end{tabular}

Table S4. Selected interactions and bond lengths $[\AA]$ and angels $\left[^{0}\right]$ of 4 (twisted)

\begin{tabular}{cccc}
\hline \multicolumn{2}{c}{ Bond Angles [ $\left.{ }^{0}\right]$} & \multicolumn{2}{c}{ Bond Length $(\AA)$} \\
\hline C1A-Au-C1C & 179.15 & Au-C1A & 2.036 \\
N1A-C1A-N1B & 109.3 & Au-C1C & 2.043 \\
N2C-C1C-N2D & 109.02 & Au-C16B & 4.006 \\
$\alpha$ & 24.44 & Au-C16D & 3.714 \\
& & C16C-C16A & 4.54 \\
\hline
\end{tabular}

Table S5. Selected interactions and bond lengths $[\AA]$ and angels $\left[{ }^{0}\right]$ of 5

\begin{tabular}{cccc}
\hline \multicolumn{2}{c}{ Bond Angles [ $\left.{ }^{0}\right]$} & \multicolumn{2}{c}{ Bond Length $(\AA)$} \\
\hline C1-Cu-C1 & 180 & Cu-C1 & 1.912 \\
N1A-C1-N1B & 108.74 & C16A-C16B & 4.26 \\
NMI-NMI $_{\text {avg }}$ & 4.63 & CO (O1'B)-C15A & 3.435 \\
$\alpha$ & 20.33 & & \\
\hline
\end{tabular}




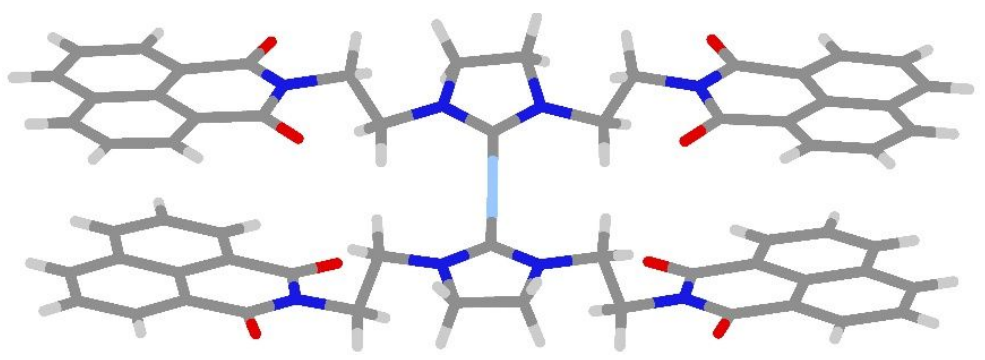

Figure S28. Molecular view of optimized geometries of $\mathbf{3}$ in Ground-State $\left(\mathrm{S}_{0}\right)$.

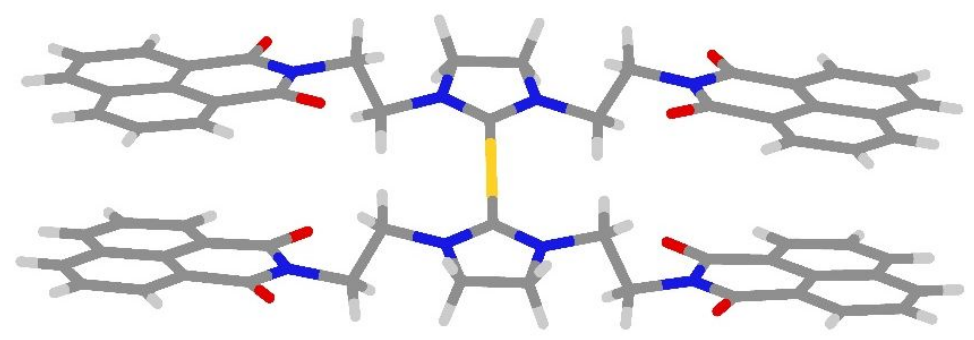

Figure S29. Molecular view of optimized geometries of $\mathbf{4}$ in Ground-State $\left(\mathrm{S}_{0}\right)$.

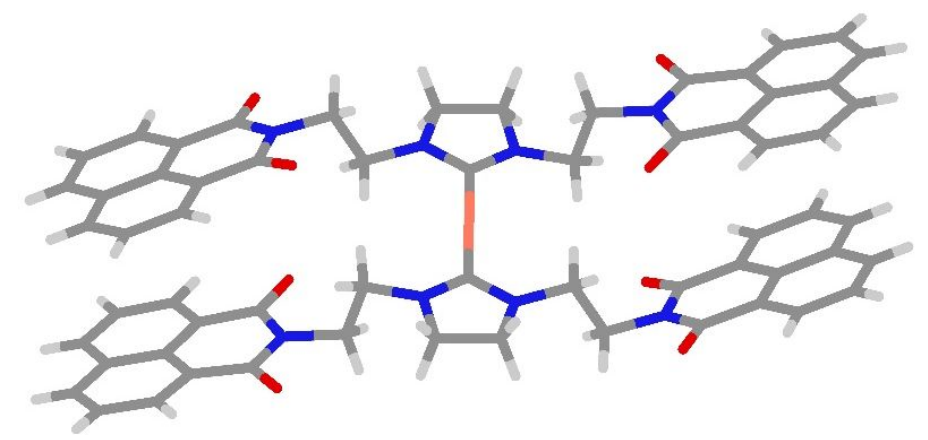

Figure S30. Molecular view of optimized geometries of 5 in Ground-State $\left(\mathrm{S}_{0}\right)$.

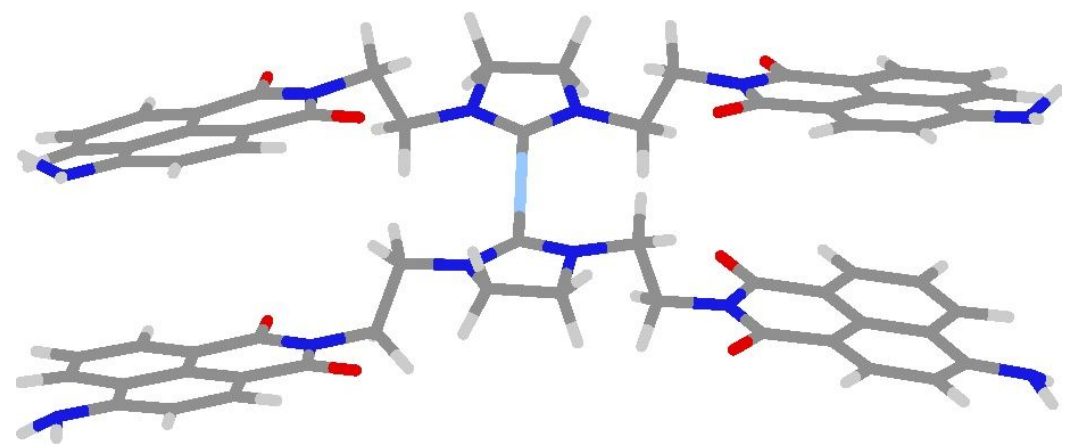

Figure S31. Molecular view of optimized geometries of 6 in ground-state $\left(\mathrm{S}_{0}\right)$. 


\section{Photophysical Properties}

Emission and excitation spectra for all compounds in solution upon exposure to the air were recorded on a FluoroMax 4 (JY Horiba Inc.) spectrofluorometer in a $1 \mathrm{~cm}$ quartz cuvette (freshly distilled dichloromethane, concentration of $\sim 10-5 \mathrm{M}, 298 \mathrm{~K}$ ). The emission quantum yields in solution were determined by a comparative method using a $365 \mathrm{~nm}$ light-emitting diode as the excitation source and Fluorescence quantum yields were calculated using quinine sulphate $(\Phi \mathrm{f}=0.54)$ in $0.1 \mathrm{~N} \mathrm{H} 2 \mathrm{SO} 4$ as standard.3 Excitation and emission spectra the solid state at $298 \mathrm{~K}$ were recorded with a HORIBA FluoroMax-4 spectrofluorometer. Lifetime and emission quantum yields in the solid phase were performed on a HORIBA Scientific FluoroLog-3 spectrofluorometer. The absolute emission quantum yields of solid samples, which were loaded in Teflon cuvettes and covered by a quartz glass ring, were measured using a FluoroLog 3 (JY Horiba Inc.) spectrofluorometer and a Quanta-phi integration sphere (Horiba).

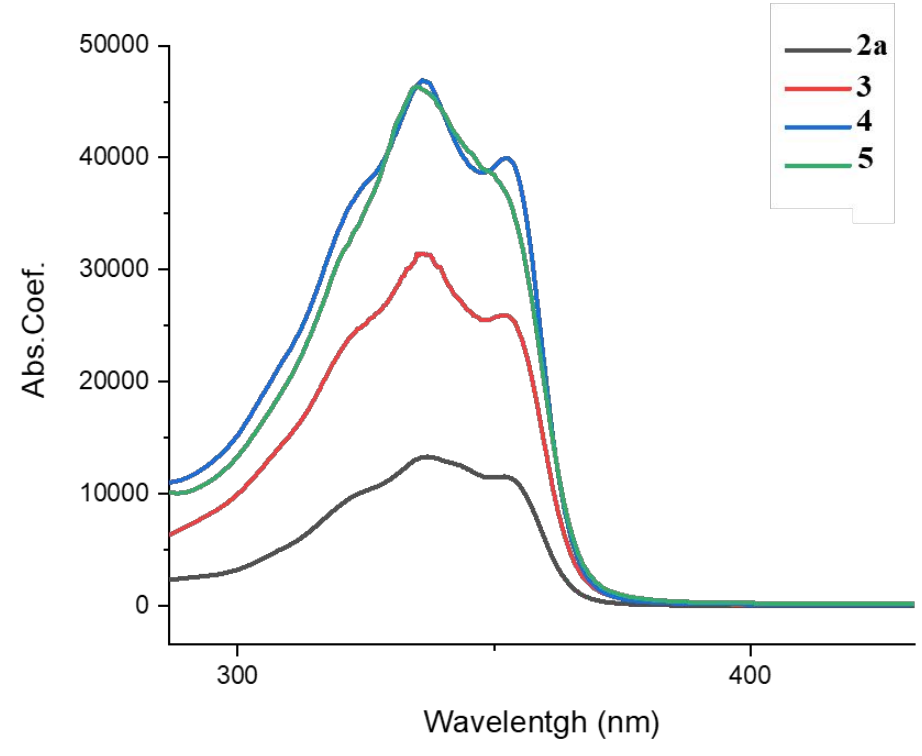

Figure S32. The absorption coefficient spectra of 2a and 3-5 in DCM.

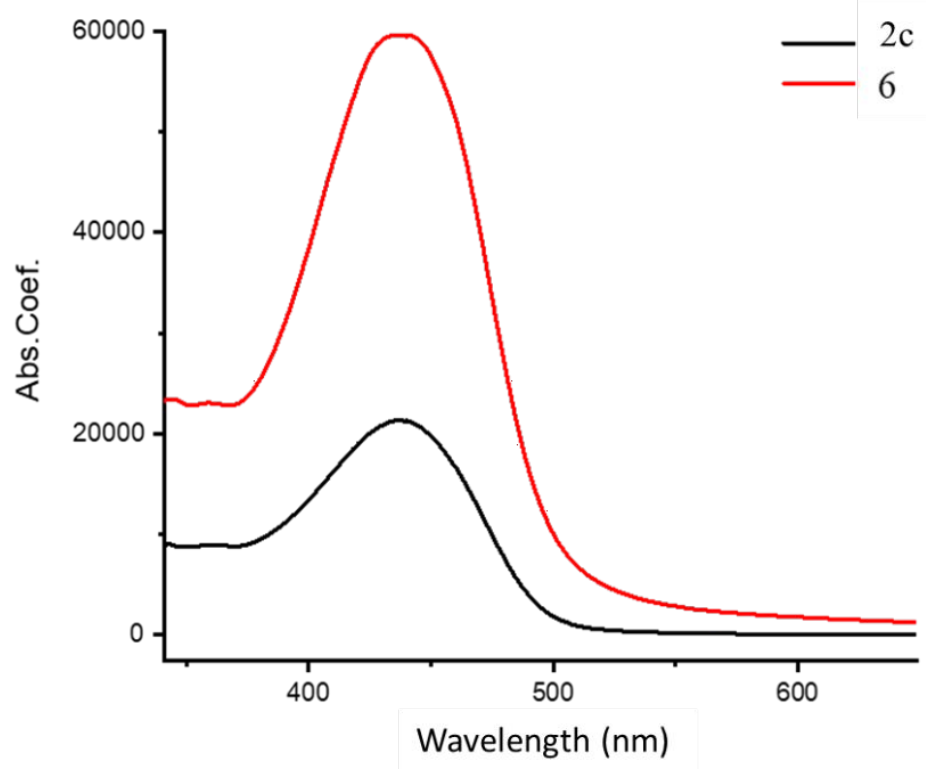

Figure S33. The absorption coefficient spectra of 2c and 6 in DMSO. 


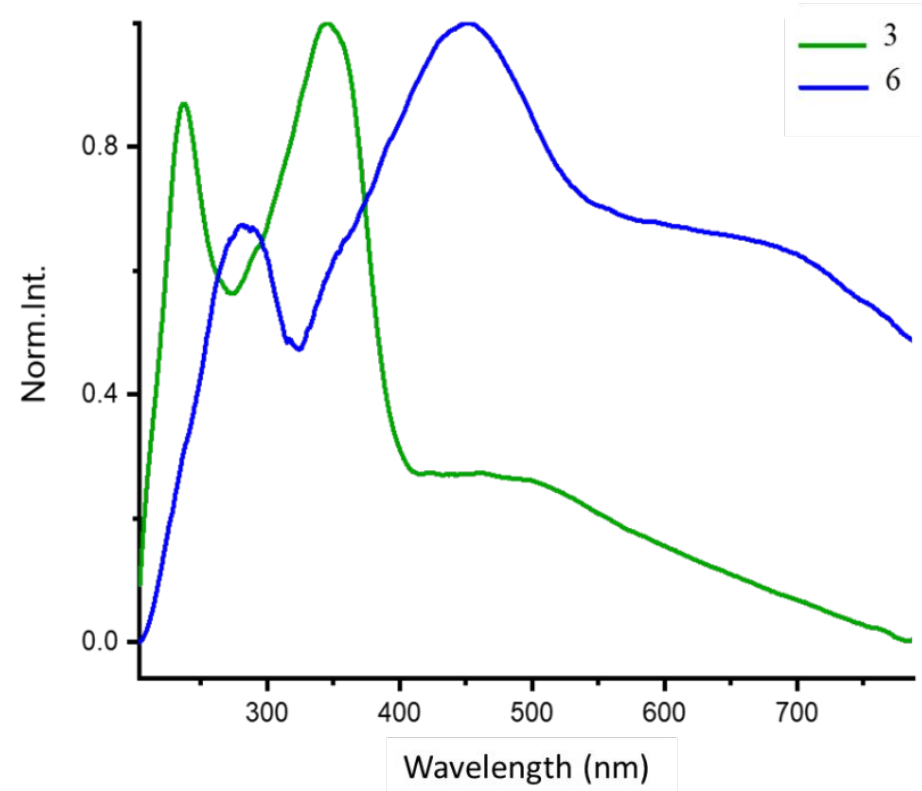

Figure S34. The absorption spectra of $\mathbf{3}$ and $\mathbf{6}$ in solid state.

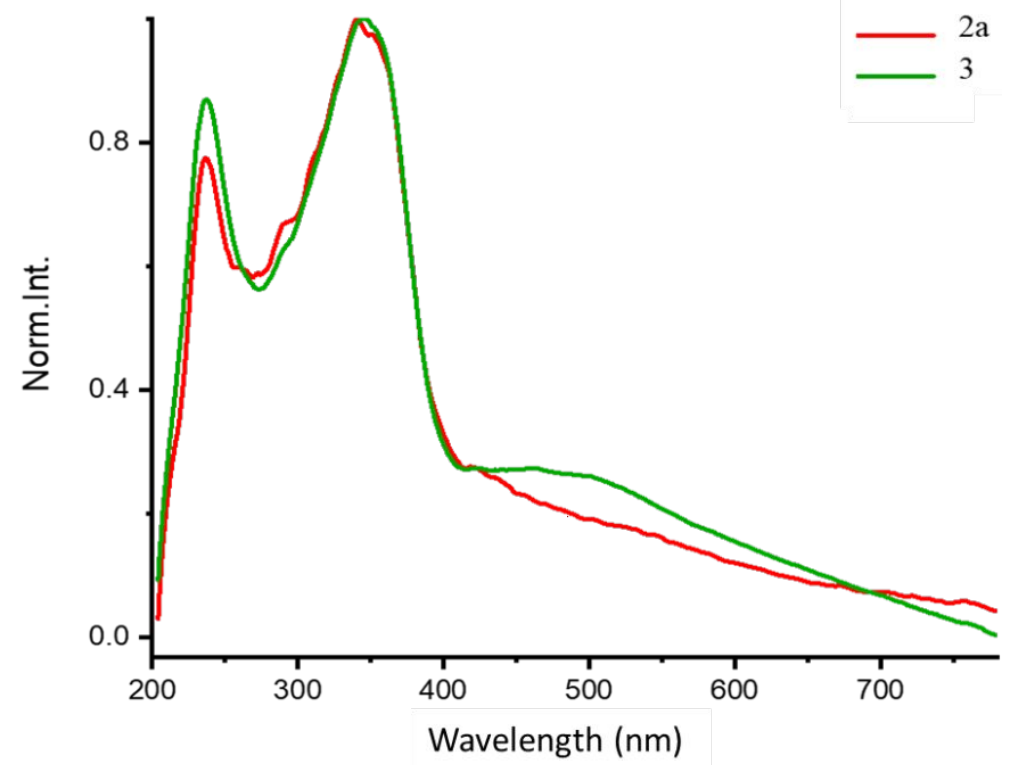

Figure S35. The absorption spectra of $\mathbf{2 a}$ and $\mathbf{3}$ in solid state. 

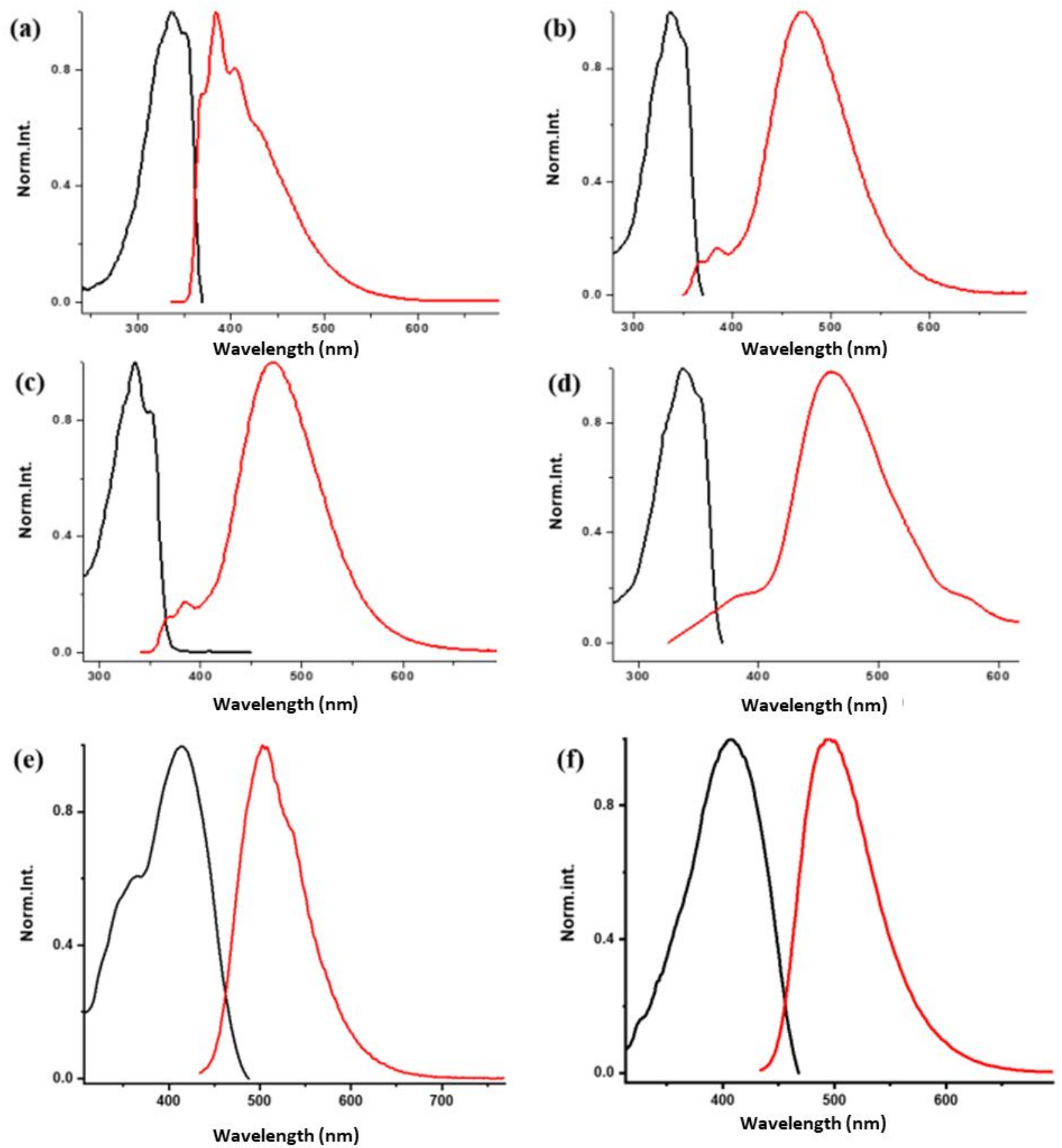

Figure S36. Excitation (black) and emission (red) spectra of a) 2a, b) 3, c) 4, d) 5, e) 2c and f) 6 in dichloromethane. 


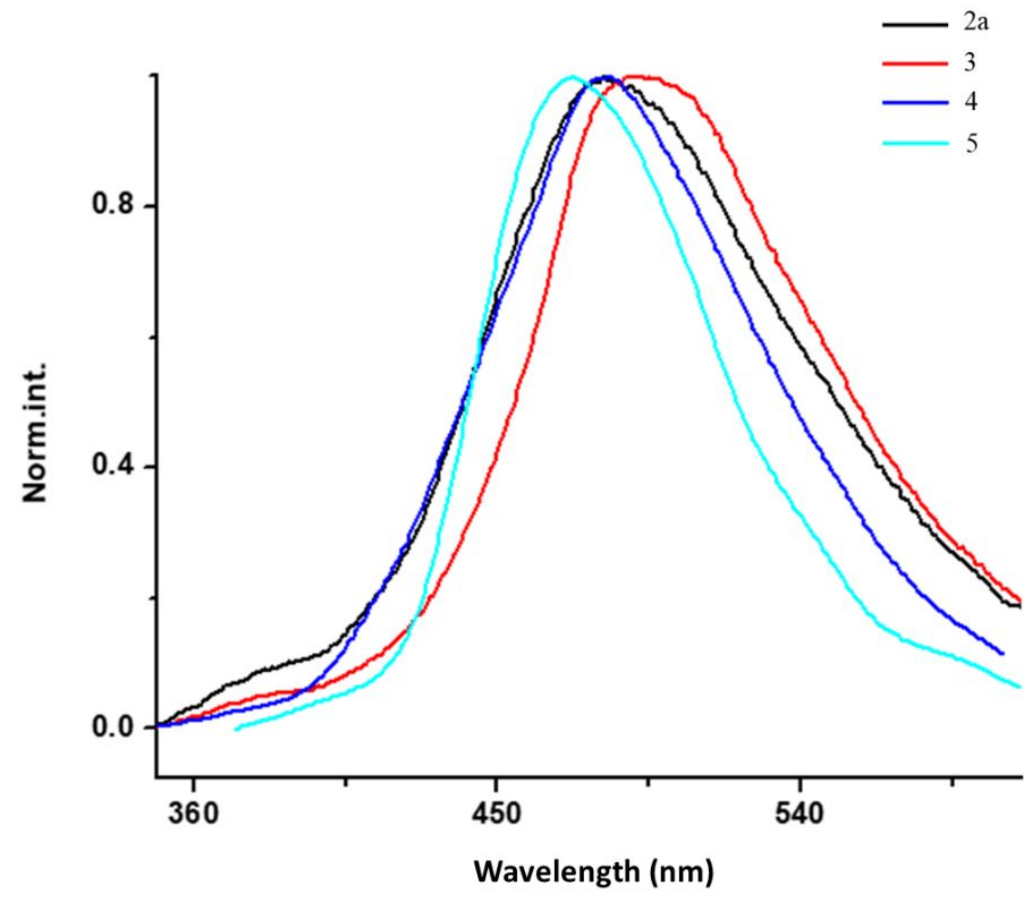

Figure S37: emission spectra of 2a and 3-5 in solid state.

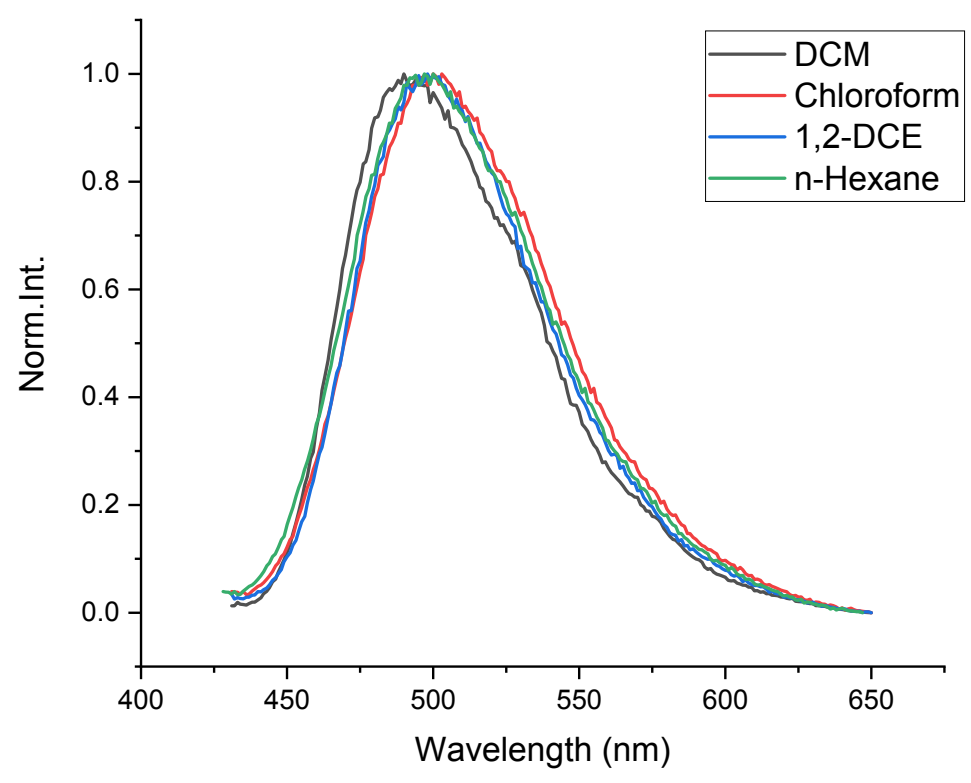

Figure S38: The change of emission spectra of $\mathbf{6}$ with changing of solvent polarity (negligible change was observed about $5 \mathrm{~nm}$ ). 


\section{Computational Details}

Compounds 2a, 2c and 3-6 were studied using the hybrid PBE0 density functional method. The gold, silver and copper atoms were described by a triple-valence $\zeta$-quality basis set with polarization functions (def2-TZVP). Scalar relativistic effects were taken into account by employing a 60-electron relativistic effective core potential. A split-valence basis set with polarization functions was used for the other atoms $\left(6-31 \mathrm{G}^{*}\right)$. The electronic transitions of the complexes were investigated by means of timedependent DFT calculations (PBE0-TDDFT). For 3-5 and 6, the first singlet excited state $\left(\mathrm{S}_{1}\right)$ geometry was calculated from the crystal structure and the optimized ground state $\left(\mathrm{S}_{0}\right)$ geometry, respectively for gas and solution state. The geometries of the lowest-energy singlet excited and ground states of each complex were used to investigate their absorption and emission features. All electronic structure calculations and geometry optimizations were performed with ORCA version 4.1.0. 
Cartesian coordinate, $\mathbf{4}, \mathrm{S} 0$, in DCM

\begin{tabular}{|c|c|c|c|c|c|c|c|}
\hline & $\mathrm{x}$ & $\mathrm{y}$ & $\mathrm{z}$ & & $\mathrm{x}$ & $\mathrm{y}$ & $\mathrm{z}$ \\
\hline $\mathrm{Au}$ & 10.777 & 6.9327 & 9.0558 & C & 7.9363 & 9.7264 & 9.8758 \\
\hline $\mathrm{C}$ & 9.1782 & 0.8912 & 8.2068 & $\mathrm{C}$ & 9.1297 & 9.3539 & 10.759 \\
\hline $\mathrm{C}$ & 8.9063 & -0.152 & 9.1194 & $\mathrm{C}$ & 9.1306 & 15.817 & 7.9808 \\
\hline $\mathrm{C}$ & 9.5983 & 0.5989 & 6.9234 & $\mathrm{C}$ & 8.3951 & 7.7082 & 12.561 \\
\hline $\mathrm{C}$ & 8.4825 & 0.1362 & 10.435 & $\mathrm{C}$ & 13.196 & 1.5304 & 7.2196 \\
\hline $\mathrm{C}$ & 8.3451 & 1.5331 & 10.878 & $\mathrm{C}$ & 12.629 & -0.156 & 8.9753 \\
\hline $\mathrm{C}$ & 8.9987 & 2.2948 & 8.6119 & $\mathrm{C}$ & 13.053 & 0.1333 & 7.66 \\
\hline $\mathrm{C}$ & 9.0546 & -1.504 & 8.707 & $\mathrm{C}$ & 12.361 & 0.8873 & 9.8898 \\
\hline $\mathrm{C}$ & 9.766 & -0.737 & 6.5156 & $\mathrm{C}$ & 12.546 & 2.2909 & 9.4869 \\
\hline $\mathrm{C}$ & 8.3251 & -2.228 & 10.908 & $\mathrm{C}$ & 13.336 & -0.891 & 6.778 \\
\hline $\mathrm{C}$ & 9.4971 & -1.768 & 7.3879 & $\mathrm{C}$ & 11.941 & 0.5945 & 11.173 \\
\hline $\mathrm{C}$ & 13.668 & 12.18 & 8.2932 & $\mathrm{C}$ & 13.2 & 3.9007 & 7.7656 \\
\hline $\mathrm{C}$ & 12.489 & 13.382 & 10.143 & $\mathrm{C}$ & 12.475 & -1.508 & 9.3855 \\
\hline $\mathrm{C}$ & 8.1951 & -0.888 & 11.315 & $\mathrm{C}$ & 11.768 & -0.741 & 11.579 \\
\hline $\mathrm{C}$ & 8.3509 & 3.9041 & 10.336 & $\mathrm{C}$ & 11.996 & 4.495 & 7.0297 \\
\hline $\mathrm{C}$ & 13.259 & 13.428 & 8.9589 & $\mathrm{C}$ & 12.032 & -1.772 & 10.704 \\
\hline $\mathrm{C}$ & 9.5548 & 4.4918 & 11.076 & $\mathrm{C}$ & 12.772 & -2.534 & 8.4569 \\
\hline $\mathrm{C}$ & 12.157 & 12.145 & 10.739 & $\mathrm{C}$ & 13.201 & -2.231 & 7.183 \\
\hline $\mathrm{C}$ & 12.598 & 10.886 & 10.118 & $\mathrm{C}$ & 13.189 & 6.1864 & 5.5664 \\
\hline $\mathrm{C}$ & 8.7533 & -2.531 & 9.6337 & $\mathrm{H}$ & 9.7914 & 1.4147 & 6.2346 \\
\hline $\mathrm{C}$ & 13.589 & 14.644 & 8.393 & $\mathrm{H}$ & 10.108 & -0.952 & 5.5082 \\
\hline $\mathrm{C}$ & 8.3657 & 6.1809 & 12.545 & $\mathrm{H}$ & 8.0866 & -3.024 & 11.605 \\
\hline $\mathrm{C}$ & 9.602 & 6.9344 & 10.728 & $\mathrm{H}$ & 9.6222 & -2.799 & 7.0687 \\
\hline $\mathrm{C}$ & 11.396 & 12.11 & 11.891 & $\mathrm{H}$ & 7.8644 & -0.641 & 12.319 \\
\hline $\mathrm{C}$ & 13.611 & 9.7369 & 8.2324 & $\mathrm{H}$ & 7.4679 & 3.9068 & 10.977 \\
\hline $\mathrm{C}$ & 12.032 & 14.592 & 10.736 & $\mathrm{H}$ & 8.1495 & 4.4853 & 9.435 \\
\hline $\mathrm{C}$ & 10.918 & 13.3 & 12.467 & $\mathrm{H}$ & 9.7621 & 3.9081 & 11.978 \\
\hline $\mathrm{C}$ & 12.418 & 9.358 & 7.3512 & $\mathrm{H}$ & 10.445 & 4.4601 & 10.444 \\
\hline $\mathrm{C}$ & 11.23 & 14.518 & 11.901 & $\mathrm{H}$ & 8.8624 & -3.567 & 9.3214 \\
\hline $\mathrm{C}$ & 12.393 & 15.821 & 10.132 & $\mathrm{H}$ & 14.175 & 14.655 & 7.4797 \\
\hline $\mathrm{C}$ & 13.164 & 15.845 & 8.99 & $\mathrm{H}$ & 8.7007 & 5.7332 & 13.483 \\
\hline $\mathrm{C}$ & 11.952 & 6.9373 & 7.3827 & $\mathrm{H}$ & 7.37 & 5.7937 & 12.3 \\
\hline $\mathrm{C}$ & 13.157 & 7.7134 & 5.5496 & $\mathrm{H}$ & 11.17 & 11.15 & 12.344 \\
\hline $\mathrm{C}$ & 9.3794 & 12.142 & 7.3703 & $\mathrm{H}$ & 13.785 & 8.9651 & 8.9819 \\
\hline $\mathrm{C}$ & 9.0424 & 13.378 & 7.9669 & $\mathrm{H}$ & 14.515 & 9.887 & 7.6413 \\
\hline $\mathrm{C}$ & 10.14 & 12.111 & 6.2171 & $\mathrm{H}$ & 10.304 & 13.254 & 13.36 \\
\hline $\mathrm{C}$ & 8.2729 & 13.419 & 9.1517 & $\mathrm{H}$ & 11.493 & 9.4332 & 7.9256 \\
\hline $\mathrm{C}$ & 7.8688 & 12.169 & 9.8163 & $\mathrm{H}$ & 12.344 & 10.053 & 6.5093 \\
\hline $\mathrm{C}$ & 8.9444 & 10.881 & 7.9901 & $\mathrm{H}$ & 10.861 & 15.438 & 12.346 \\
\hline $\mathrm{C}$ & 9.4947 & 14.589 & 7.3752 & $\mathrm{H}$ & 12.046 & 16.746 & 10.586 \\
\hline $\mathrm{C}$ & 10.612 & 13.304 & 5.6419 & $\mathrm{H}$ & 13.443 & 16.791 & 8.5364 \\
\hline $\mathrm{C}$ & 8.3601 & 15.836 & 9.1239 & $\mathrm{H}$ & 14.15 & 8.1628 & 5.4851 \\
\hline $\mathrm{C}$ & 10.296 & 14.52 & 6.2093 & $\mathrm{H}$ & 12.539 & 8.1128 & 4.7371 \\
\hline $\mathrm{C}$ & 7.9392 & 14.633 & 9.7193 & $\mathrm{H}$ & 10.37 & 11.153 & 5.7636 \\
\hline
\end{tabular}




\begin{tabular}{|c|c|c|c|c|c|c|c|}
\hline \multicolumn{3}{|c|}{ Cartesian coordinate, $\mathbf{4}, \mathrm{S} 0$, in $\mathrm{DCM}$} & \multicolumn{5}{|c|}{ Cartesian coordinate, $\mathbf{3}, \mathrm{S} 0$, in DCM } \\
\hline \multicolumn{3}{|c|}{$\begin{array}{ll}x & y\end{array}$} & \multirow{2}{*}{$\frac{z}{4.748}$} & & \multirow{2}{*}{$\begin{array}{c}\mathrm{x} \\
3.0053\end{array}$} & \multirow{2}{*}{$\frac{\mathrm{y}}{13.6725}$} & \multirow{2}{*}{$\frac{\mathrm{Z}}{5.9792}$} \\
\hline $\mathrm{H}$ & 11.226 & 13.261 & & $\mathrm{Ag}$ & & & \\
\hline $\mathrm{H}$ & 8.0788 & 16.781 & 9.5793 & $\mathrm{C}$ & 1.3822 & 19.3896 & 7.6575 \\
\hline $\mathrm{H}$ & 10.661 & 15.442 & 5.7653 & $\mathrm{C}$ & 0.961 & 18.0793 & 7.3349 \\
\hline $\mathrm{H}$ & 7.3539 & 14.641 & 10.633 & $\mathrm{C}$ & 4.0145 & 13.1823 & 7.7764 \\
\hline $\mathrm{H}$ & 7.031 & 9.8717 & 10.465 & $\mathrm{C}$ & 2.3989 & 19.6076 & 8.6163 \\
\hline $\mathrm{H}$ & 7.7682 & 8.9536 & 9.126 & $\mathrm{C}$ & 0.4801 & 7.3933 & 1.353 \\
\hline $\mathrm{H}$ & 9.1998 & 10.051 & 11.6 & $\mathrm{C}$ & 1.509 & 7.607 & 2.3005 \\
\hline $\mathrm{H}$ & 10.055 & 9.4322 & 10.186 & $\mathrm{C}$ & 3.9834 & 20.9115 & 4.7341 \\
\hline $\mathrm{H}$ & 9.4742 & 16.743 & 7.5286 & $\mathrm{C}$ & -0.1721 & 8.4851 & 0.7375 \\
\hline $\mathrm{H}$ & 9.0107 & 8.1089 & 13.375 & $\mathrm{C}$ & 1.5917 & 16.9247 & 7.9928 \\
\hline $\mathrm{H}$ & 7.4007 & 8.1551 & 12.624 & $\mathrm{C}$ & 4.3234 & 15.4726 & 8.629 \\
\hline $\mathrm{H}$ & 13.667 & -0.643 & 5.775 & $\mathrm{C}$ & 1.6914 & 6.857 & 6.9916 \\
\hline $\mathrm{H}$ & 11.752 & 1.4098 & 11.863 & $\mathrm{C}$ & 3.6864 & 19.5928 & 4.152 \\
\hline $\mathrm{H}$ & 13.406 & 4.4791 & 8.6672 & $\mathrm{C}$ & 5.0345 & 18.5215 & 5.9376 \\
\hline $\mathrm{H}$ & 14.081 & 3.9003 & 7.1222 & $\mathrm{C}$ & 2.6438 & 16.663 & 4.4997 \\
\hline $\mathrm{H}$ & 11.426 & -0.956 & 12.586 & $\mathrm{C}$ & 2.2114 & 21.9957 & 8.278 \\
\hline $\mathrm{H}$ & 11.108 & 4.4641 & 7.6632 & $\mathrm{C}$ & 0.6485 & 7.0636 & 6.0593 \\
\hline $\mathrm{H}$ & 11.785 & 3.9151 & 6.1264 & $\mathrm{C}$ & 3.0635 & 18.4652 & 9.2646 \\
\hline $\mathrm{H}$ & 11.903 & -2.803 & 11.022 & $\mathrm{C}$ & 0.7932 & 20.5002 & 6.9915 \\
\hline $\mathrm{H}$ & 12.659 & -3.57 & 8.7675 & $\mathrm{C}$ & -0.0151 & 17.8753 & 6.3764 \\
\hline $\mathrm{H}$ & 13.436 & -3.027 & 6.4842 & $\mathrm{C}$ & 2.1319 & 6.5316 & 2.9012 \\
\hline $\mathrm{H}$ & 14.185 & 5.8016 & 5.8125 & $\mathrm{C}$ & 3.843 & 10.7507 & 7.5182 \\
\hline $\mathrm{H}$ & 12.857 & 5.7374 & 4.6273 & $\mathrm{C}$ & 1.927 & 8.9785 & 2.6415 \\
\hline $\mathrm{N}$ & 8.5853 & 2.5258 & 9.9243 & $\mathrm{C}$ & 2.8004 & 20.8931 & 8.9234 \\
\hline $\mathrm{N}$ & 9.3043 & 5.8618 & 11.465 & $\mathrm{C}$ & 4.8149 & 21.012 & 5.8716 \\
\hline $\mathrm{N}$ & 13.315 & 10.985 & 8.9271 & $\mathrm{C}$ & 2.3338 & 8.0084 & 7.6428 \\
\hline $\mathrm{N}$ & 12.516 & 8.0149 & 6.8339 & $\mathrm{C}$ & -0.2029 & 20.2552 & 6.0149 \\
\hline $\mathrm{N}$ & 8.2268 & 10.976 & 9.1815 & $\mathrm{C}$ & 0.7542 & 4.9859 & 1.6518 \\
\hline $\mathrm{N}$ & 9.0365 & 8.0111 & 11.278 & $\mathrm{C}$ & 1.2357 & 21.8046 & 7.3237 \\
\hline $\mathrm{N}$ & 12.96 & 2.5224 & 8.1748 & $\mathrm{C}$ & 0.0861 & 6.0667 & 1.0252 \\
\hline $\mathrm{N}$ & 12.25 & 5.8655 & 6.6449 & $\mathrm{C}$ & 5.3307 & 19.8521 & 6.4923 \\
\hline $\mathrm{O}$ & 8.0271 & 1.8435 & 12.016 & $\mathrm{C}$ & 4.0335 & 17.1906 & 4.1401 \\
\hline $\mathrm{O}$ & 9.1906 & 3.229 & 7.8451 & $\mathrm{C}$ & 4.8615 & 11.9004 & 9.54 \\
\hline $\mathrm{O}$ & 14.271 & 12.155 & 7.2311 & $\mathrm{C}$ & -0.601 & 18.9695 & 5.716 \\
\hline $\mathrm{O}$ & 12.33 & 9.7865 & 10.587 & $\mathrm{C}$ & 0.2566 & 9.8624 & 1.0323 \\
\hline $\mathrm{O}$ & 7.266 & 12.14 & 10.878 & $\mathrm{C}$ & 0.0108 & 5.9428 & 5.4616 \\
\hline $\mathrm{O}$ & 9.2173 & 9.783 & 7.5208 & $\mathrm{C}$ & 1.333 & 14.3077 & 1.937 \\
\hline $\mathrm{O}$ & 13.514 & 1.841 & 6.081 & $\mathrm{C}$ & 2.1012 & 5.5769 & 7.31 \\
\hline \multirow[t]{6}{*}{$\mathrm{O}$} & 12.358 & 3.2246 & 10.255 & $\mathrm{C}$ & 0.2368 & 8.371 & 5.7121 \\
\hline & & & & $\mathrm{C}$ & -1.1939 & 8.2668 & -0.1675 \\
\hline & & & & $\mathrm{C}$ & 3.2291 & 16.04 & 9.5435 \\
\hline & & & & $\mathrm{C}$ & -0.9606 & 5.8779 & 0.0898 \\
\hline & & & & $\mathrm{C}$ & 1.7565 & 5.2157 & 2.5683 \\
\hline & & & & $\mathrm{C}$ & 2.1943 & 14.2637 & 4.1127 \\
\hline
\end{tabular}




\begin{tabular}{|c|c|c|c|c|c|c|c|}
\hline \multicolumn{8}{|c|}{ Cartesian coordinate, $\mathbf{3}, \mathrm{S} 0$, in DCM } \\
\hline & $\mathrm{x}$ & $\mathrm{y}$ & $\mathrm{z}$ & & $\mathrm{x}$ & $\mathrm{y}$ & Z \\
\hline $\mathrm{C}$ & 6.1065 & 19.9585 & 7.6303 & $\mathrm{H}$ & 2.259 & 4.3828 & 3.0488 \\
\hline $\mathrm{C}$ & 5.9401 & 22.3669 & 7.5619 & $\mathrm{H}$ & 6.4701 & 19.0546 & 8.1061 \\
\hline $\mathrm{C}$ & 5.1192 & 22.2922 & 6.4101 & $\mathrm{H}$ & 6.1831 & 23.3428 & 7.9741 \\
\hline $\mathrm{C}$ & 3.443 & 22.0477 & 4.1644 & $\mathrm{H}$ & 2.7947 & 21.9416 & 3.3003 \\
\hline $\mathrm{C}$ & 0.8776 & 9.5381 & 6.3397 & $\mathrm{H}$ & -2.3942 & 6.7987 & -1.2045 \\
\hline $\mathrm{C}$ & -1.5909 & 6.9571 & -0.4915 & $\mathrm{H}$ & 2.4519 & 10.148 & 9.0694 \\
\hline $\mathrm{C}$ & 2.4373 & 10.4001 & 8.0065 & $\mathrm{H}$ & 1.7757 & 11.2512 & 7.844 \\
\hline $\mathrm{C}$ & 0.4521 & 4.6428 & 5.8114 & $\mathrm{H}$ & -0.0328 & 3.787 & 5.3487 \\
\hline $\mathrm{C}$ & 1.8907 & 11.3433 & 2.0627 & $\mathrm{H}$ & 1.7016 & 11.8529 & 1.1173 \\
\hline $\mathrm{C}$ & 1.2771 & 12.1355 & 3.2124 & $\mathrm{H}$ & 2.967 & 11.2369 & 2.2094 \\
\hline $\mathrm{C}$ & 1.6505 & 15.7378 & 2.3724 & $\mathrm{H}$ & 0.1823 & 12.0726 & 3.1502 \\
\hline $\mathrm{C}$ & -0.7762 & 8.5587 & 4.7914 & $\mathrm{H}$ & 1.5861 & 11.7331 & 4.1789 \\
\hline $\mathrm{C}$ & -1.0299 & 6.1712 & 4.5285 & $\mathrm{H}$ & 2.3579 & 16.2318 & 1.7018 \\
\hline $\mathrm{C}$ & 1.4782 & 4.4642 & 6.7152 & $\mathrm{H}$ & 0.7575 & 16.3669 & 2.4577 \\
\hline $\mathrm{C}$ & 4.5715 & 23.4389 & 5.7865 & $\mathrm{H}$ & -1.0766 & 9.5702 & 4.5362 \\
\hline $\mathrm{C}$ & 3.7403 & 23.3172 & 4.6931 & $\mathrm{H}$ & -1.5184 & 5.3174 & 4.0688 \\
\hline $\mathrm{C}$ & -1.4133 & 7.4525 & 4.1994 & $\mathrm{H}$ & 1.8137 & 3.4657 & 6.9782 \\
\hline $\mathrm{C}$ & 5.0634 & 13.3836 & 9.8548 & $\mathrm{H}$ & 4.8142 & 24.4177 & 6.1926 \\
\hline $\mathrm{C}$ & 6.4176 & 21.2229 & 8.1626 & $\mathrm{H}$ & 3.3129 & 24.2005 & 4.2291 \\
\hline $\mathrm{H}$ & 4.1129 & 15.7312 & 7.5902 & $\mathrm{H}$ & -2.2061 & 7.6173 & 3.4765 \\
\hline $\mathrm{H}$ & 5.2941 & 15.9144 & 8.8768 & $\mathrm{H}$ & 6.12 & 13.6706 & 9.8928 \\
\hline $\mathrm{H}$ & 2.6102 & 16.363 & 5.5495 & $\mathrm{H}$ & 4.5864 & 13.6862 & 10.7904 \\
\hline $\mathrm{H}$ & 1.9101 & 17.4621 & 4.354 & $\mathrm{H}$ & 7.0344 & 21.2932 & 9.0527 \\
\hline $\mathrm{H}$ & 2.5431 & 22.9978 & 8.5297 & $\mathrm{~N}$ & 2.5864 & 17.1943 & 8.9297 \\
\hline $\mathrm{H}$ & -0.3175 & 16.8607 & 6.1357 & $\mathrm{~N}$ & 4.2748 & 18.4841 & 4.7675 \\
\hline $\mathrm{H}$ & 2.9137 & 6.7203 & 3.6301 & $\mathrm{~N}$ & 1.864 & 9.2723 & 7.2916 \\
\hline $\mathrm{H}$ & 4.5366 & 9.9324 & 7.7265 & $\mathrm{~N}$ & 4.3202 & 11.9443 & 8.174 \\
\hline $\mathrm{H}$ & 3.8327 & 10.9249 & 6.4366 & $\mathrm{~N}$ & 1.3254 & 10.0102 & 1.9192 \\
\hline $\mathrm{H}$ & 3.5871 & 21.0351 & 9.6567 & $\mathrm{~N}$ & 1.667 & 13.525 & 3.1353 \\
\hline $\mathrm{H}$ & -0.6514 & 21.1005 & 5.5006 & $\mathrm{~N}$ & 2.2425 & 15.5342 & 3.7 \\
\hline $\mathrm{H}$ & 0.457 & 3.9719 & 1.3962 & $\mathrm{~N}$ & 4.4182 & 14.0385 & 8.7116 \\
\hline $\mathrm{H}$ & 0.7924 & 22.653 & 6.8089 & $\mathrm{O}$ & 3.2299 & 7.9003 & 8.4684 \\
\hline $\mathrm{H}$ & 4.8078 & 16.5038 & 4.4831 & $\mathrm{O}$ & 2.9709 & 19.4367 & 3.1729 \\
\hline $\mathrm{H}$ & 4.1212 & 17.3274 & 3.0603 & $\mathrm{O}$ & 1.2923 & 15.7646 & 7.7315 \\
\hline $\mathrm{H}$ & 4.1432 & 11.4287 & 10.2181 & $\mathrm{O}$ & 2.775 & 9.2376 & 3.4834 \\
\hline $\mathrm{H}$ & 5.7905 & 11.3257 & 9.5677 & $\mathrm{O}$ & 5.4359 & 17.4864 & 6.452 \\
\hline $\mathrm{H}$ & -1.3653 & 18.7927 & 4.9658 & $\mathrm{O}$ & 3.9888 & 18.589 & 10.0521 \\
\hline $\mathrm{H}$ & 0.2833 & 14.1563 & 1.6662 & $\mathrm{O}$ & 0.5594 & 10.6909 & 6.0788 \\
\hline $\mathrm{H}$ & 1.9548 & 13.9944 & 1.0948 & $\mathrm{O}$ & -0.2624 & 10.8455 & 0.5226 \\
\hline $\mathrm{H}$ & 2.9061 & 5.4449 & 8.0263 & & & & \\
\hline $\mathrm{H}$ & -1.679 & 9.121 & -0.6279 & & & & \\
\hline $\mathrm{H}$ & 2.4603 & 15.2841 & 9.7081 & & & & \\
\hline $\mathrm{H}$ & 3.6348 & 16.3645 & 10.5006 & & & & \\
\hline $\mathrm{H}$ & -1.2645 & 4.8658 & -0.1647 & & & & \\
\hline
\end{tabular}


Cartesian coordinate, $\mathbf{5}, \mathrm{S} 0$, in DCM

\begin{tabular}{|c|c|c|c|c|c|c|c|}
\hline & $\mathrm{x}$ & $\mathrm{y}$ & $\mathrm{z}$ & & $\mathrm{x}$ & $\mathrm{y}$ & Z \\
\hline $\mathrm{C}$ & 6.8588 & 8.9772 & 0.1153 & $\mathrm{C}$ & 7.7776 & 8.9362 & 10.164 \\
\hline $\mathrm{C}$ & 6.7331 & 8.2642 & 1.3944 & $\mathrm{C}$ & 10.186 & 9.0888 & 2.8271 \\
\hline $\mathrm{C}$ & 5.4195 & 2.8262 & 11.465 & $\mathrm{C}$ & 10.935 & 11.76 & -1.4694 \\
\hline $\mathrm{C}$ & 4.1797 & 4.9329 & 11.681 & $\mathrm{C}$ & 8.1245 & 10.658 & 4.6785 \\
\hline $\mathrm{C}$ & 7.6822 & 5.8157 & 5.6871 & $\mathrm{C}$ & 11.129 & 10.443 & -1.8275 \\
\hline $\mathrm{C}$ & 6.9362 & 6.8365 & -1.0852 & $\mathrm{C}$ & 7.1611 & 11.341 & 8.217 \\
\hline $\mathrm{C}$ & 4.5022 & 3.6444 & 12.161 & $\mathrm{C}$ & 9.6341 & 10.778 & 4.4823 \\
\hline $\mathrm{C}$ & 6.8258 & 6.1034 & 0.1873 & $\mathrm{C}$ & 11.042 & 7.0649 & -0.2363 \\
\hline $\mathrm{C}$ & 5.928 & 3.2486 & 10.151 & $\mathrm{C}$ & 11.171 & 8.0411 & -1.1993 \\
\hline $\mathrm{C}$ & 3.9082 & 3.1701 & 13.362 & $\mathrm{C}$ & 10.569 & 12.093 & -0.1534 \\
\hline $\mathrm{C}$ & 6.9478 & 8.2498 & -1.0923 & $\mathrm{C}$ & 5.9772 & 5.9666 & 14.668 \\
\hline $\mathrm{C}$ & 6.8801 & 10.358 & 0.1003 & $\mathrm{C}$ & 5.0025 & 8.5557 & 14.176 \\
\hline $\mathrm{C}$ & 3.2715 & 5.7212 & 12.362 & $\mathrm{C}$ & 7.4073 & 4.1199 & 14.003 \\
\hline $\mathrm{C}$ & 6.5303 & 6.1671 & 2.6088 & $\mathrm{C}$ & 6.49 & 4.6631 & 14.875 \\
\hline $\mathrm{C}$ & 4.8021 & 5.437 & 10.445 & $\mathrm{C}$ & 6.724 & 8.9989 & 9.0598 \\
\hline $\mathrm{C}$ & 5.2417 & 1.1461 & 13.195 & $\mathrm{C}$ & 5.0319 & 6.5626 & 15.537 \\
\hline $\mathrm{C}$ & 7.3484 & 5.0467 & 8.0166 & $\mathrm{C}$ & 4.5543 & 7.8325 & 15.295 \\
\hline $\mathrm{C}$ & 4.302 & 1.9038 & 13.86 & $\mathrm{C}$ & 7.6245 & 11.866 & 6.8534 \\
\hline $\mathrm{C}$ & 7.6575 & 3.7809 & 4.5434 & $\mathrm{Cu}$ & 7.6086 & 7.7053 & 6.1238 \\
\hline $\mathrm{C}$ & 5.8682 & 4.8315 & 8.3204 & $\mathrm{H}$ & 6.8084 & 10.895 & 1.0413 \\
\hline $\mathrm{C}$ & 2.6471 & 5.2383 & 13.526 & $\mathrm{H}$ & 3.0419 & 6.7104 & 11.981 \\
\hline $\mathrm{C}$ & 2.9611 & 3.991 & 14.02 & $\mathrm{H}$ & 6.0172 & 5.2268 & 2.4046 \\
\hline $\mathrm{C}$ & 5.7929 & 1.6019 & 11.984 & $\mathrm{H}$ & 5.9181 & 6.7838 & 3.2684 \\
\hline $\mathrm{C}$ & 7.0484 & 8.9482 & -2.3266 & $\mathrm{H}$ & 5.5542 & 0.1874 & 13.596 \\
\hline $\mathrm{C}$ & 7.0211 & 6.1357 & -2.2725 & $\mathrm{H}$ & 7.6833 & 6.0464 & 8.2962 \\
\hline $\mathrm{C}$ & 6.9807 & 11.054 & -1.1172 & $\mathrm{H}$ & 7.96 & 4.3301 & 8.5677 \\
\hline $\mathrm{C}$ & 7.0604 & 10.364 & -2.3069 & $\mathrm{H}$ & 3.8498 & 1.5383 & 14.778 \\
\hline $\mathrm{C}$ & 7.9009 & 5.9356 & 3.2352 & $\mathrm{H}$ & 8.455 & 3.2817 & 3.9883 \\
\hline $\mathrm{C}$ & 7.1254 & 8.1989 & -3.5256 & $\mathrm{H}$ & 6.6953 & 3.4974 & 4.1000 \\
\hline $\mathrm{C}$ & 7.111 & 6.8214 & -3.4976 & $\mathrm{H}$ & 5.293 & 5.7185 & 8.0532 \\
\hline $\mathrm{C}$ & 7.7102 & 3.5069 & 6.0437 & $\mathrm{H}$ & 5.5046 & 3.9924 & 7.7193 \\
\hline $\mathrm{C}$ & 7.3447 & 6.1158 & 12.636 & $\mathrm{H}$ & 1.9287 & 5.8642 & 14.045 \\
\hline $\mathrm{C}$ & 7.7933 & 6.8596 & 11.447 & $\mathrm{H}$ & 2.4898 & 3.6244 & 14.928 \\
\hline $\mathrm{C}$ & 10.422 & 11.105 & 0.8009 & $\mathrm{H}$ & 6.5031 & 0.9918 & 11.434 \\
\hline $\mathrm{C}$ & 10.515 & 8.7351 & 1.4363 & $\mathrm{H}$ & 7.0098 & 5.0501 & -2.2419 \\
\hline $\mathrm{C}$ & 7.4728 & 9.5326 & 6.7635 & $\mathrm{H}$ & 6.9944 & 12.14 & -1.1147 \\
\hline $\mathrm{C}$ & 5.9258 & 8.0038 & 13.308 & $\mathrm{H}$ & 7.1354 & 10.901 & -3.2489 \\
\hline $\mathrm{C}$ & 10.636 & 9.7508 & 0.4606 & $\mathrm{H}$ & 8.521 & 5.3465 & 2.551 \\
\hline $\mathrm{C}$ & 6.4005 & 8.7837 & 12.155 & $\mathrm{H}$ & 8.4012 & 6.8906 & 3.4044 \\
\hline $\mathrm{C}$ & 10.032 & 11.472 & 2.172 & $\mathrm{H}$ & 7.1971 & 8.7291 & -4.4718 \\
\hline $\mathrm{C}$ & 10.98 & 9.4061 & -0.8755 & $\mathrm{H}$ & 7.1699 & 6.2568 & -4.4228 \\
\hline $\mathrm{C}$ & 6.4202 & 6.6996 & 13.533 & $\mathrm{H}$ & 6.9122 & 2.8458 & 6.3892 \\
\hline $\mathrm{C}$ & 7.8273 & 4.8415 & 12.871 & $\mathrm{H}$ & 8.6693 & 3.0832 & 6.3642 \\
\hline $\mathrm{C}$ & 10.722 & 7.4129 & 1.0885 & $\mathrm{H}$ & 8.5318 & 4.4077 & 12.168 \\
\hline
\end{tabular}


Cartesian coordinate, 5, S0, in DCM

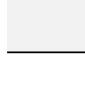

Cartesian coordinate, $\mathbf{6}, \mathrm{S} 0$, in DCM

\begin{tabular}{|c|c|c|c|c|c|c|c|}
\hline & $\mathrm{x}$ & $\mathrm{y}$ & $\mathrm{z}$ & & $\mathrm{x}$ & $\mathrm{y}$ & Z \\
\hline $\mathrm{H}$ & 10.641 & 6.6462 & 1.8519 & $\mathrm{Ag}$ & 10.793 & 6.4828 & 8.8314 \\
\hline $\mathrm{H}$ & 8.0086 & 9.9403 & 10.524 & $\mathrm{C}$ & 9.4893 & 0.6446 & 8.0169 \\
\hline $\mathrm{H}$ & 8.6888 & 8.4681 & 9.7934 & $\mathrm{C}$ & 8.8639 & -0.4136 & 8.7135 \\
\hline $\mathrm{H}$ & 11.064 & 12.548 & -2.2043 & $\mathrm{C}$ & 10.3832 & 0.3487 & 6.9964 \\
\hline $\mathrm{H}$ & 7.7674 & 9.7377 & 4.2134 & $\mathrm{C}$ & 7.9262 & -0.1343 & 9.7354 \\
\hline $\mathrm{H}$ & 7.6153 & 11.492 & 4.1837 & $\mathrm{C}$ & 7.629 & 1.2591 & 10.1098 \\
\hline $\mathrm{H}$ & 11.407 & 10.183 & -2.8453 & $\mathrm{C}$ & 9.219 & 2.0296 & 8.3738 \\
\hline $\mathrm{H}$ & 6.1634 & 11.693 & 8.4987 & $\mathrm{C}$ & 9.1628 & -1.7664 & 8.3894 \\
\hline $\mathrm{H}$ & 7.857 & 11.604 & 9.0179 & $\mathrm{C}$ & 10.6958 & -0.9644 & 6.6644 \\
\hline $\mathrm{H}$ & 10.148 & 10.075 & 5.1383 & $\mathrm{C}$ & 7.5621 & -2.4958 & 10.0696 \\
\hline $\mathrm{H}$ & 9.9922 & 11.789 & 4.6751 & $\mathrm{C}$ & 10.1309 & -2.0352 & 7.3598 \\
\hline $\mathrm{H}$ & 11.2 & 6.0223 & -0.4947 & $\mathrm{C}$ & 13.1468 & 11.8922 & 7.2661 \\
\hline $\mathrm{H}$ & 11.432 & 7.774 & -2.2197 & $\mathrm{C}$ & 12.6309 & 13.5005 & 9.102 \\
\hline $\mathrm{H}$ & 10.401 & 13.127 & 0.1324 & $\mathrm{C}$ & 7.2869 & -1.1624 & 10.4042 \\
\hline $\mathrm{H}$ & 4.6314 & 9.5563 & 13.98 & $\mathrm{C}$ & 8.0804 & 3.6357 & 9.8055 \\
\hline $\mathrm{H}$ & 7.7964 & 3.1221 & 14.176 & $\mathrm{C}$ & 13.0476 & 13.2538 & 7.7761 \\
\hline $\mathrm{H}$ & 6.1471 & 4.0949 & 15.736 & $\mathrm{C}$ & 9.2063 & 4.099 & 10.7264 \\
\hline $\mathrm{H}$ & 5.7757 & 9.3494 & 9.4781 & $\mathrm{C}$ & 12.336 & 12.4201 & 9.9654 \\
\hline $\mathrm{H}$ & 6.5482 & 8.0098 & 8.6306 & $\mathrm{C}$ & 12.4691 & 11.033 & 9.4907 \\
\hline $\mathrm{H}$ & 4.6821 & 6 & 16.398 & $\mathrm{C}$ & 8.4847 & -2.7907 & 9.0836 \\
\hline $\mathrm{H}$ & 3.8282 & 8.2802 & 15.967 & $\mathrm{C}$ & 13.3529 & 14.3243 & 6.9499 \\
\hline $\mathrm{H}$ & 8.5898 & 12.379 & 6.9088 & $\mathrm{C}$ & 8.0268 & 5.8209 & 12.1486 \\
\hline $\mathrm{H}$ & 6.9008 & 12.542 & 6.3878 & $\mathrm{C}$ & 9.533 & 6.5358 & 10.5245 \\
\hline $\mathrm{N}$ & 7.5647 & 4.8539 & 6.6034 & $\mathrm{C}$ & 11.9208 & 12.6505 & 11.2633 \\
\hline $\mathrm{N}$ & 5.5622 & 4.5203 & 9.7103 & $\mathrm{C}$ & 13.0366 & 9.5005 & 7.6762 \\
\hline $\mathrm{N}$ & 7.8279 & 5.2359 & 4.4919 & $\mathrm{C}$ & 12.4993 & 14.8324 & 9.5841 \\
\hline $\mathrm{N}$ & 6.6763 & 6.8743 & 1.3426 & $\mathrm{C}$ & 11.7863 & 13.9623 & 11.7392 \\
\hline $\mathrm{N}$ & 7.7395 & 10.637 & 6.066 & $\mathrm{C}$ & 11.8341 & 9.0292 & 6.8559 \\
\hline $\mathrm{N}$ & 9.9874 & 10.437 & 3.1089 & $\mathrm{C}$ & 12.0701 & 15.0299 & 10.9144 \\
\hline $\mathrm{N}$ & 7.1384 & 9.8899 & 8.0054 & $\mathrm{C}$ & 12.79 & 15.9171 & 8.6909 \\
\hline $\mathrm{N}$ & 7.3093 & 8.159 & 11.301 & $\mathrm{C}$ & 13.2392 & 15.6356 & 7.3998 \\
\hline $\mathrm{O}$ & 6.6684 & 8.8417 & 2.4721 & $\mathrm{C}$ & 11.9117 & 6.5776 & 7.0431 \\
\hline $\mathrm{O}$ & 6.8514 & 4.8838 & 0.2637 & $\mathrm{C}$ & 12.8416 & 7.4941 & 5.1019 \\
\hline $\mathrm{O}$ & 4.6415 & 6.5807 & 10.042 & $\mathrm{C}$ & 9.0758 & 12.7337 & 8.2042 \\
\hline $\mathrm{O}$ & 6.617 & 2.5286 & 9.4398 & $\mathrm{C}$ & 8.9426 & 13.7546 & 9.1728 \\
\hline $\mathrm{O}$ & 8.5631 & 6.3896 & 10.619 & $\mathrm{C}$ & 9.4674 & 13.0747 & 6.9165 \\
\hline $\mathrm{O}$ & 6.0442 & 9.9321 & 11.929 & $\mathrm{C}$ & 8.505 & 13.4426 & 10.4797 \\
\hline $\mathrm{O}$ & 10.084 & 8.2549 & 3.7203 & $\mathrm{C}$ & 8.2433 & 12.0441 & 10.8575 \\
\hline \multirow[t]{5}{*}{$\mathrm{O}$} & 9.758 & 12.616 & 2.5042 & $\mathrm{C}$ & 8.8041 & 11.3421 & 8.5449 \\
\hline & & & & $\mathrm{C}$ & 9.2342 & 15.1065 & 8.844 \\
\hline & & & & $\mathrm{C}$ & 9.7759 & 14.3881 & 6.5754 \\
\hline & & & & $\mathrm{C}$ & 8.5667 & 15.7775 & 11.0827 \\
\hline & & & & $\mathrm{C}$ & 9.708 & 15.4126 & 7.5221 \\
\hline
\end{tabular}


Cartesian coordinate, $\mathbf{6}, \mathrm{S} 0$, in DCM

\begin{tabular}{|c|c|c|c|c|c|c|c|}
\hline & $\mathrm{x}$ & $\mathrm{y}$ & $\mathrm{z}$ & & $\mathrm{x}$ & $\mathrm{y}$ & Z \\
\hline $\mathrm{C}$ & 8.0967 & 9.7031 & 10.2117 & $\mathrm{H}$ & 7.9639 & 14.1671 & 12.4072 \\
\hline $\mathrm{C}$ & 9.3367 & 8.996 & 10.7624 & $\mathrm{H}$ & 7.2996 & 9.7196 & 10.9568 \\
\hline $\mathrm{C}$ & 9.0307 & 16.1014 & 9.8225 & $\mathrm{H}$ & 7.7493 & 9.1935 & 9.3128 \\
\hline $\mathrm{C}$ & 8.2833 & 7.31 & 12.3311 & $\mathrm{H}$ & 9.7023 & 9.5254 & 11.6496 \\
\hline $\mathrm{C}$ & 13.9007 & 1.526 & 7.8012 & $\mathrm{H}$ & 10.1387 & 8.9995 & 10.0213 \\
\hline $\mathrm{C}$ & 12.7167 & 0.1216 & 9.4957 & $\mathrm{H}$ & 9.2159 & 17.1437 & 9.5786 \\
\hline $\mathrm{C}$ & 13.5541 & 0.2244 & 8.3624 & $\mathrm{H}$ & 8.9116 & 7.5266 & 13.2042 \\
\hline $\mathrm{C}$ & 12.2846 & 1.2926 & 10.1614 & $\mathrm{H}$ & 7.3657 & 7.8948 & 12.4041 \\
\hline $\mathrm{C}$ & 12.676 & 2.619 & 9.6544 & $\mathrm{H}$ & 14.6386 & -0.8444 & 6.8711 \\
\hline $\mathrm{C}$ & 13.9922 & -0.9358 & 7.7385 & $\mathrm{H}$ & 11.157 & 2.1211 & 11.7682 \\
\hline $\mathrm{C}$ & 11.4581 & 1.2081 & 11.267 & $\mathrm{H}$ & 13.5955 & 4.707 & 8.6485 \\
\hline $\mathrm{C}$ & 13.6364 & 3.9544 & 7.861 & $\mathrm{H}$ & 14.6195 & 3.9594 & 7.3891 \\
\hline $\mathrm{C}$ & 12.288 & -1.1496 & 9.9734 & $\mathrm{H}$ & 10.347 & -0.0991 & 12.5795 \\
\hline $\mathrm{C}$ & 11.0158 & -0.0397 & 11.7266 & $\mathrm{H}$ & 11.5619 & 3.9387 & 7.2914 \\
\hline $\mathrm{C}$ & 12.5227 & 4.2007 & 6.8422 & $\mathrm{H}$ & 12.6765 & 3.5538 & 5.9732 \\
\hline $\mathrm{C}$ & 11.4245 & -1.1946 & 11.0896 & $\mathrm{H}$ & 11.0564 & -2.1449 & 11.4631 \\
\hline $\mathrm{C}$ & 12.74 & -2.3295 & 9.2846 & $\mathrm{H}$ & 13.9569 & -3.0828 & 7.6861 \\
\hline $\mathrm{C}$ & 13.6058 & -2.1896 & 8.1948 & $\mathrm{H}$ & 14.1209 & 5.6987 & 5.0523 \\
\hline $\mathrm{C}$ & 13.0641 & 5.9773 & 5.0988 & $\mathrm{H}$ & 12.5369 & 5.4768 & 4.2808 \\
\hline $\mathrm{H}$ & 10.8527 & 1.1682 & 6.4631 & $\mathrm{H}$ & 10.4973 & -3.9796 & 7.8108 \\
\hline $\mathrm{H}$ & 11.4272 & -1.1713 & 5.8886 & $\mathrm{H}$ & 11.2594 & -3.4198 & 6.4284 \\
\hline $\mathrm{H}$ & 7.0414 & -3.2986 & 10.5811 & $\mathrm{H}$ & 12.8023 & -4.3579 & 9.275 \\
\hline $\mathrm{H}$ & 6.568 & -0.9153 & 11.1795 & $\mathrm{H}$ & 11.9141 & -3.7108 & 10.5603 \\
\hline $\mathrm{H}$ & 7.1148 & 3.6725 & 10.3109 & $\mathrm{H}$ & 12.532 & 17.4318 & 10.0485 \\
\hline $\mathrm{H}$ & 8.0538 & 4.2536 & 8.906 & $\mathrm{H}$ & 12.995 & 17.9262 & 8.5081 \\
\hline $\mathrm{H}$ & 9.232 & 3.461 & 11.6167 & $\mathrm{H}$ & 10.5116 & 16.8061 & 6.3108 \\
\hline $\mathrm{H}$ & 10.1678 & 4.0087 & 10.2178 & $\mathrm{H}$ & 10.4822 & 17.2472 & 7.9271 \\
\hline $\mathrm{H}$ & 8.6601 & -3.8295 & 8.8208 & $\mathrm{~N}$ & 8.3084 & 2.2529 & 9.4097 \\
\hline $\mathrm{H}$ & 13.6868 & 14.1196 & 5.9376 & $\mathrm{~N}$ & 9.0442 & 5.4644 & 11.1577 \\
\hline $\mathrm{H}$ & 8.1592 & 5.2413 & 13.0653 & $\mathrm{~N}$ & 12.8668 & 10.8581 & 8.1692 \\
\hline $\mathrm{H}$ & 7.0271 & 5.6237 & 11.7401 & $\mathrm{~N}$ & 12.0801 & 7.7004 & 6.3415 \\
\hline $\mathrm{H}$ & 11.7038 & 11.8021 & 11.9039 & $\mathrm{~N}$ & 8.3877 & 11.0846 & 9.8584 \\
\hline $\mathrm{H}$ & 13.1677 & 8.8501 & 8.5414 & $\mathrm{~N}$ & 9.038 & 7.6286 & 11.1118 \\
\hline $\mathrm{H}$ & 13.9402 & 9.4761 & 7.0642 & $\mathrm{~N}$ & 13.4256 & 2.6533 & 8.4853 \\
\hline $\mathrm{H}$ & 11.4502 & 14.1388 & 12.7558 & $\mathrm{~N}$ & 12.4759 & 5.5696 & 6.3812 \\
\hline $\mathrm{H}$ & 10.9274 & 9.0238 & 7.4678 & $\mathrm{~N}$ & 10.4594 & -3.3232 & 7.0407 \\
\hline $\mathrm{H}$ & 11.6631 & 9.7037 & 6.0134 & $\mathrm{~N}$ & 12.2875 & -3.5649 & 9.6339 \\
\hline $\mathrm{H}$ & 11.946 & 16.033 & 11.3099 & $\mathrm{~N}$ & 12.543 & 17.2105 & 9.0627 \\
\hline $\mathrm{H}$ & 13.4804 & 16.4602 & 6.7353 & $\mathrm{~N}$ & 10.0371 & 16.6987 & 7.1982 \\
\hline $\mathrm{H}$ & 13.7795 & 8.0575 & 5.1255 & $\mathrm{O}$ & 6.8351 & 1.5574 & 10.9925 \\
\hline $\mathrm{H}$ & 12.2542 & 7.8396 & 4.2465 & $\mathrm{O}$ & 9.7447 & 2.992 & 7.8192 \\
\hline $\mathrm{H}$ & 9.5509 & 12.2886 & 6.1723 & $\mathrm{O}$ & 13.4637 & 11.6164 & 6.1142 \\
\hline
\end{tabular}




\begin{tabular}{llllllll} 
& 10.1162 & 14.6217 & 5.5705 & $\mathrm{O}$ & 12.2479 & 10.0683 & 10.2142 \\
$\mathrm{H}$ & 8.3924 & 16.5602 & 11.814 & $\mathrm{O}$ & 7.91 & 11.7144 & 11.9887 \\
\hline
\end{tabular}

\begin{tabular}{|c|c|c|c|c|c|c|c|}
\hline \multicolumn{3}{|c|}{ Cartesian coordinate, $\mathbf{6}, \mathrm{S} 0$, in DCM } & \multicolumn{5}{|c|}{ Cartesian coordinate, $\mathbf{4}, \mathrm{S} 1$, Gas State } \\
\hline & $\mathrm{x}$ & $\mathrm{y}$ & \multirow{2}{*}{$\begin{array}{c}\mathrm{z} \\
7.7513\end{array}$} & \multicolumn{2}{|c|}{$\mathrm{x}$} & \multirow{2}{*}{$\frac{\mathrm{y}}{6.7078}$} & \multirow{2}{*}{$\frac{Z}{9.0556}$} \\
\hline $\mathrm{O}$ & 8.9185 & 10.4125 & & $\mathrm{Au}$ & 10.9553 & & \\
\hline $\mathrm{O}$ & 14.5515 & 1.6753 & 6.7729 & $\mathrm{O}$ & 7.472816 & 1.80391 & 11.71112 \\
\hline \multirow[t]{39}{*}{$\mathrm{O}$} & 12.3406 & 3.6612 & 10.2093 & $\mathrm{O}$ & 9.669564 & 2.538007 & 7.808038 \\
\hline & & & & $\mathrm{O}$ & 12.28002 & 11.0278 & 10.39708 \\
\hline & & & & $\mathrm{O}$ & 14.58872 & 11.41369 & 6.511152 \\
\hline & & & & $\mathrm{N}$ & 8.486476 & 2.153533 & 9.716086 \\
\hline & & & & $\mathrm{N}$ & 9.0021 & 5.6206 & 11.0538 \\
\hline & & & & $\mathrm{N}$ & 13.42041 & 11.23385 & 8.453282 \\
\hline & & & & $\mathrm{C}$ & 9.193757 & 0.263702 & 8.313409 \\
\hline & & & & $\mathrm{C}$ & 8.674544 & -0.60793 & 9.287171 \\
\hline & & & & $\mathrm{C}$ & 9.747336 & -0.221 & 7.162648 \\
\hline & & & & $\mathrm{H}$ & 10.11052 & 0.367861 & 6.541065 \\
\hline & & & & $\mathrm{C}$ & 8.074065 & -0.13529 & 10.47781 \\
\hline & & & & $\mathrm{C}$ & 7.960602 & 1.32368 & 10.73636 \\
\hline & & & & $\mathrm{C}$ & 9.170455 & 1.703079 & 8.559365 \\
\hline & & & & $\mathrm{C}$ & 8.737596 & -2.0241 & 9.041273 \\
\hline & & & & $\mathrm{N}$ & 12.867 & 7.7733 & 7.0268 \\
\hline & & & & $\mathrm{C}$ & 9.772076 & -1.61347 & 6.909794 \\
\hline & & & & $\mathrm{H}$ & 10.11295 & -1.94485 & 6.110513 \\
\hline & & & & $\mathrm{C}$ & 7.622602 & -2.4093 & 11.18061 \\
\hline & & & & $\mathrm{H}$ & 7.281924 & -3.00575 & 11.80756 \\
\hline & & & & $\mathrm{C}$ & 9.294522 & -2.4439 & 7.845655 \\
\hline & & & & $\mathrm{H}$ & 9.339885 & -3.35833 & 7.682204 \\
\hline & & & & $\mathrm{C}$ & 12.795 & 11.77785 & 9.583158 \\
\hline & & & & $\mathrm{C}$ & 13.46127 & 14.03207 & 8.733852 \\
\hline & & & & $\mathrm{C}$ & 7.530867 & -1.00596 & 11.41253 \\
\hline & & & & $\mathrm{H}$ & 7.114235 & -0.67925 & 12.17697 \\
\hline & & & & $\mathrm{C}$ & 8.2554 & 3.5972 & 9.8919 \\
\hline & & & & $\mathrm{H}$ & 7.399613 & 3.734633 & 10.32733 \\
\hline & & & & $\mathrm{H}$ & 8.22077 & 4.024989 & 9.022094 \\
\hline & & & & $\mathrm{C}$ & 12.81885 & 13.21677 & 9.715816 \\
\hline & & & & $\mathrm{C}$ & 9.3515 & 4.2387 & 10.7242 \\
\hline & & & & $\mathrm{H}$ & 9.4771 & 3.7319 & 11.5417 \\
\hline & & & & $\mathrm{H}$ & 10.1856 & 4.224 & 10.2293 \\
\hline & & & & $\mathrm{C}$ & 14.12439 & 13.45449 & 7.639657 \\
\hline & & & & $\mathrm{C}$ & 14.07829 & 11.98043 & 7.463822 \\
\hline & & & & $\mathrm{C}$ & 8.206994 & -2.8795 & 10.04491 \\
\hline & & & & $\mathrm{H}$ & 8.261576 & -3.79948 & 9.920221 \\
\hline & & & & $\mathrm{C}$ & 12.1819 & 13.79343 & 10.81468 \\
\hline & & & & $\mathrm{H}$ & 11.77055 & 13.26259 & 11.45796 \\
\hline & & & & $\mathrm{C}$ & 8.0278 & 5.9505 & 12.1264 \\
\hline
\end{tabular}




\begin{tabular}{llll}
$\mathrm{H}$ & 8.3172 & 5.5998 & 12.9832 \\
$\mathrm{H}$ & 7.1472 & 5.5997 & 11.9204 \\
$\mathrm{C}$ & 9.5638 & 6.7078 & 10.4446 \\
\hline
\end{tabular}

\begin{tabular}{|c|c|c|c|c|c|c|c|}
\hline \multicolumn{8}{|c|}{ Cartesian coordinate, $\mathbf{4}, \mathrm{S} 1$, Gas State } \\
\hline & $\mathrm{x}$ & $\mathrm{y}$ & $\mathrm{z}$ & & $\mathrm{x}$ & $\mathrm{y}$ & Z \\
\hline $\mathrm{C}$ & 14.7474 & 14.23132 & 6.743406 & $\mathrm{C}$ & 9.3515 & 9.1769 & 10.7242 \\
\hline $\mathrm{H}$ & 15.19966 & 13.84878 & 6.026464 & $\mathrm{H}$ & 10.18572 & 9.191666 & 10.22934 \\
\hline $\mathrm{C}$ & 13.4415 & 9.773 & 8.296 & $\mathrm{H}$ & 9.476978 & 9.683734 & 11.54166 \\
\hline $\mathrm{H}$ & 13.29657 & 9.410441 & 9.18395 & $\mathrm{C}$ & 10.12761 & 15.75499 & 8.025221 \\
\hline $\mathrm{H}$ & 14.34607 & 9.550268 & 8.025537 & $\mathrm{H}$ & 10.32888 & 16.65521 & 7.907057 \\
\hline $\mathrm{C}$ & 13.42221 & 15.4518 & 8.890523 & $\mathrm{C}$ & 8.0278 & 7.4651 & 12.1264 \\
\hline $\mathrm{C}$ & 14.71257 & 15.63897 & 6.895112 & $\mathrm{H}$ & 8.3172 & 7.8159 & 12.9832 \\
\hline $\mathrm{H}$ & 15.13334 & 16.1796 & 6.26616 & $\mathrm{H}$ & 7.1472 & 7.8159 & 11.9204 \\
\hline $\mathrm{C}$ & 12.4466 & 9.1338 & 7.3174 & $\mathrm{O}$ & 11.94447 & 2.582744 & 10.4684 \\
\hline $\mathrm{H}$ & 11.5588 & 9.1274 & 7.7082 & $\mathrm{O}$ & 13.88239 & 1.510375 & 6.509391 \\
\hline $\mathrm{H}$ & 12.4139 & 9.6504 & 6.4971 & $\mathrm{~N}$ & 12.89648 & 2.037541 & 8.489094 \\
\hline $\mathrm{C}$ & 14.09429 & 16.18846 & 7.911284 & $\mathrm{~N}$ & 12.867 & 5.6424 & 7.0268 \\
\hline $\mathrm{H}$ & 14.10325 & 17.11549 & 7.984619 & $\mathrm{C}$ & 12.20492 & 1.70446 & 9.66148 \\
\hline $\mathrm{C}$ & 12.77258 & 15.98935 & 10.01739 & $\mathrm{C}$ & 12.21459 & -0.66493 & 8.866071 \\
\hline $\mathrm{H}$ & 12.75864 & 16.91245 & 10.13025 & $\mathrm{C}$ & 11.85649 & 0.313846 & 9.843585 \\
\hline $\mathrm{C}$ & 12.1755 & 15.21851 & 10.93009 & $\mathrm{C}$ & 12.95306 & -0.30848 & 7.726599 \\
\hline $\mathrm{H}$ & 11.74777 & 15.61595 & 11.65397 & $\mathrm{C}$ & 13.28721 & 1.120877 & 7.501029 \\
\hline $\mathrm{C}$ & 12.3043 & 6.7078 & 7.6092 & $\mathrm{C}$ & 11.14375 & -0.04842 & 10.98662 \\
\hline $\mathrm{C}$ & 13.8256 & 7.472 & 5.9894 & $\mathrm{H}$ & 10.91739 & 0.586974 & 11.62676 \\
\hline $\mathrm{H}$ & 14.7027 & 7.8254 & 6.2054 & $\mathrm{C}$ & 13.30664 & -1.24303 & 6.834098 \\
\hline $\mathrm{H}$ & 13.5411 & 7.8254 & 5.132 & $\mathrm{H}$ & 13.80868 & -1.01028 & 6.086656 \\
\hline $\mathrm{O}$ & 9.479289 & 10.78734 & 7.63557 & $\mathrm{C}$ & 13.29284 & 3.43706 & 8.279805 \\
\hline $\mathrm{O}$ & 7.40679 & 11.69086 & 11.57093 & $\mathrm{H}$ & 13.29017 & 3.850442 & 9.157312 \\
\hline $\mathrm{N}$ & 8.5318 & 11.243 & 9.6417 & $\mathrm{H}$ & 14.20954 & 3.411566 & 7.963549 \\
\hline $\mathrm{N}$ & 9.0021 & 7.7951 & 11.0538 & $\mathrm{C}$ & 11.81168 & -2.01996 & 9.074023 \\
\hline $\mathrm{C}$ & 8.442419 & 13.54416 & 10.49974 & $\mathrm{C}$ & 12.91079 & -2.58757 & 7.036468 \\
\hline $\mathrm{C}$ & 9.115966 & 13.96558 & 9.339529 & $\mathrm{H}$ & 13.1443 & -3.23463 & 6.410623 \\
\hline $\mathrm{C}$ & 8.109474 & 14.43542 & 11.47972 & $\mathrm{C}$ & 12.4466 & 4.2818 & 7.3174 \\
\hline $\mathrm{H}$ & 7.640312 & 14.14502 & 12.22835 & $\mathrm{H}$ & 11.5588 & 4.2882 & 7.7082 \\
\hline $\mathrm{C}$ & 9.494794 & 13.06253 & 8.318459 & $\mathrm{H}$ & 12.4139 & 3.7652 & 6.4971 \\
\hline $\mathrm{C}$ & 9.197785 & 11.6122 & 8.447418 & $\mathrm{C}$ & 12.21922 & -2.93049 & 8.095219 \\
\hline $\mathrm{C}$ & 8.064715 & 12.1399 & 10.63478 & $\mathrm{H}$ & 11.98793 & -3.82513 & 8.200734 \\
\hline $\mathrm{C}$ & 9.449233 & 15.35919 & 9.209988 & $\mathrm{C}$ & 11.09832 & -2.3405 & 10.24414 \\
\hline $\mathrm{C}$ & 8.473688 & 15.79836 & 11.36343 & $\mathrm{H}$ & 10.84797 & -3.22422 & 10.38984 \\
\hline $\mathrm{H}$ & 8.28296 & 16.40052 & 12.04595 & $\mathrm{C}$ & 10.7689 & -1.41828 & 11.15228 \\
\hline $\mathrm{C}$ & 10.49495 & 14.87245 & 7.056403 & $\mathrm{H}$ & 10.28697 & -1.67163 & 11.90616 \\
\hline $\mathrm{H}$ & 10.94423 & 15.17489 & 6.300314 & $\mathrm{C}$ & 13.8256 & 5.9436 & 5.9894 \\
\hline $\mathrm{C}$ & 9.098446 & 16.2025 & 10.25002 & $\mathrm{H}$ & 14.7027 & 5.5903 & 6.2054 \\
\hline $\mathrm{H}$ & 9.309448 & 17.10482 & 10.17084 & $\mathrm{H}$ & 13.5411 & 5.5903 & 5.132 \\
\hline $\mathrm{C}$ & 10.19357 & 13.48649 & 7.196415 & & & & \\
\hline
\end{tabular}




\begin{tabular}{cccc}
$\mathrm{H}$ & 10.46045 & 12.8765 & 6.547248 \\
$\mathrm{C}$ & 8.2554 & 9.8185 & 9.8919 \\
$\mathrm{H}$ & 8.181203 & 9.352202 & 9.044601 \\
$\mathrm{H}$ & 7.407997 & 9.732598 & 10.356 \\
\hline
\end{tabular}

Cartesian coordinate, 3, S1, Gas State

\begin{tabular}{|c|c|c|c|c|c|c|c|}
\hline & $\mathrm{x}$ & $\mathrm{y}$ & $\mathrm{z}$ & & $\mathrm{x}$ & $\mathrm{y}$ & $z$ \\
\hline $\mathrm{Ag}$ & 10.8689 & 6.6764 & 9.1809 & $\mathrm{C}$ & 8.218 & 9.7688 & 9.9598 \\
\hline $\mathrm{C}$ & 9.2211 & 0.5439 & 8.1153 & $\mathrm{C}$ & 9.2589 & 9.1291 & 10.876 \\
\hline $\mathrm{C}$ & 8.7337 & -0.4681 & 8.9743 & $\mathrm{C}$ & 8.7776 & 16.1649 & 9.3834 \\
\hline $\mathrm{C}$ & 9.8312 & 0.2091 & 6.9227 & $\mathrm{C}$ & 7.8923 & 7.4771 & 12.25 \\
\hline $\mathrm{C}$ & 8.1052 & -0.1345 & 10.1954 & $\mathrm{C}$ & 13.5987 & 1.3154 & 7.5386 \\
\hline $\mathrm{C}$ & 7.9146 & 1.2799 & 10.5675 & $\mathrm{C}$ & 12.7485 & -0.4644 & 9.0783 \\
\hline $\mathrm{C}$ & 9.0818 & 1.9642 & 8.4861 & $\mathrm{C}$ & 13.3474 & -0.1034 & 7.8501 \\
\hline $\mathrm{C}$ & 8.882 & -1.8343 & 8.6092 & $\mathrm{C}$ & 12.3754 & 0.5259 & 10.0159 \\
\hline $\mathrm{C}$ & 9.9647 & -1.1398 & 6.5479 & $\mathrm{C}$ & 12.6055 & 1.9538 & 9.7242 \\
\hline $\mathrm{C}$ & 7.8067 & -2.4817 & 10.6907 & $\mathrm{C}$ & 13.7066 & -1.0749 & 6.9363 \\
\hline $\mathrm{C}$ & 9.5005 & -2.1407 & 7.3724 & $\mathrm{C}$ & 11.7879 & 0.1639 & 11.2126 \\
\hline $\mathrm{C}$ & 13.4153 & 12.1869 & 7.6143 & $\mathrm{C}$ & 13.5397 & 3.6458 & 8.223 \\
\hline $\mathrm{C}$ & 12.6096 & 13.9578 & 9.2019 & $\mathrm{C}$ & 12.5138 & -1.836 & 9.3701 \\
\hline $\mathrm{C}$ & 7.6511 & -1.128 & 11.0408 & $\mathrm{C}$ & 11.5507 & -1.191 & 11.5055 \\
\hline $\mathrm{C}$ & 8.1103 & 3.6343 & 9.9787 & $\mathrm{C}$ & 12.4698 & 4.2761 & 7.3297 \\
\hline $\mathrm{C}$ & 13.1525 & 13.609 & 7.955 & $\mathrm{C}$ & 11.9028 & -2.17 & 10.603 \\
\hline $\mathrm{C}$ & 9.17 & 4.2233 & 10.9111 & $\mathrm{C}$ & 12.9001 & -2.8068 & 8.414 \\
\hline $\mathrm{C}$ & 12.2797 & 12.9505 & 10.1253 & $\mathrm{C}$ & 13.484 & -2.4339 & 7.2232 \\
\hline $\mathrm{C}$ & 12.5423 & 11.5206 & 9.8124 & $\mathrm{C}$ & 13.6981 & 6.0027 & 5.9261 \\
\hline $\mathrm{C}$ & 8.4056 & -2.828 & 9.4991 & $\mathrm{H}$ & 10.2021 & 1.0031 & 6.2831 \\
\hline $\mathrm{C}$ & 13.4631 & 14.6004 & 7.0144 & $\mathrm{H}$ & 10.4365 & -1.3878 & 5.6026 \\
\hline $\mathrm{C}$ & 7.8629 & 5.9409 & 12.2598 & $\mathrm{H}$ & 7.4417 & -3.2537 & 11.3606 \\
\hline $\mathrm{C}$ & 9.4331 & 6.6729 & 10.6929 & $\mathrm{H}$ & 7.1682 & -0.8401 & 11.9695 \\
\hline $\mathrm{C}$ & 11.7008 & 13.2842 & 11.3579 & $\mathrm{H}$ & 7.1255 & 3.6756 & 10.4495 \\
\hline $\mathrm{C}$ & 13.4384 & 9.8399 & 8.2624 & $\mathrm{H}$ & 8.0951 & 4.1896 & 9.0397 \\
\hline $\mathrm{C}$ & 12.373 & 15.3197 & 9.5208 & $\mathrm{H}$ & 9.2231 & 3.6335 & 11.8326 \\
\hline $\mathrm{C}$ & 11.4683 & 14.6158 & 11.6786 & $\mathrm{H}$ & 10.1557 & 4.1892 & 10.4397 \\
\hline $\mathrm{C}$ & 12.3716 & 9.1839 & 7.3793 & $\mathrm{H}$ & 8.5127 & -3.8745 & 9.2238 \\
\hline $\mathrm{C}$ & 11.799 & 15.6307 & 10.7722 & $\mathrm{H}$ & 13.8722 & 14.2959 & 6.0574 \\
\hline $\mathrm{C}$ & 12.7044 & 16.2979 & 8.5659 & $\mathrm{H}$ & 8.1467 & 5.512 & 13.2272 \\
\hline $\mathrm{C}$ & 13.249 & 15.9352 & 7.3249 & $\mathrm{H}$ & 6.8812 & 5.5396 & 11.9884 \\
\hline $\mathrm{C}$ & 12.2514 & 6.7217 & 7.6155 & $\mathrm{H}$ & 11.4374 & 12.4845 & 12.0407 \\
\hline $\mathrm{C}$ & 13.6689 & 7.5374 & 5.9427 & $\mathrm{H}$ & 13.5199 & 9.3068 & 9.2103 \\
\hline $\mathrm{C}$ & 9.2834 & 12.7392 & 7.9586 & $\mathrm{H}$ & 14.4101 & 9.8408 & 7.7629 \\
\hline $\mathrm{C}$ & 8.9398 & 13.7785 & 8.8601 & $\mathrm{H}$ & 11.0063 & 14.8714 & 12.624 \\
\hline $\mathrm{C}$ & 9.7485 & 13.0491 & 6.6617 & $\mathrm{H}$ & 11.4018 & 9.1805 & 7.8811 \\
\hline $\mathrm{C}$ & 8.4758 & 13.4881 & 10.1693 & $\mathrm{H}$ & 12.2469 & 9.7546 & 6.4542 \\
\hline $\mathrm{C}$ & 8.2597 & 12.1152 & 10.5723 & $\mathrm{H}$ & 11.5973 & 16.6661 & 11.0254 \\
\hline $\mathrm{C}$ & 9.1533 & 11.3579 & 8.3646 & $\mathrm{H}$ & 13.4899 & 16.7062 & 6.6017 \\
\hline
\end{tabular}




\begin{tabular}{cccccccc}
$\mathrm{C}$ & 9.0731 & 15.148 & 8.4456 & $\mathrm{H}$ & 14.6556 & 7.9715 & 6.1344 \\
$\mathrm{C}$ & 9.8296 & 14.3716 & 6.2538 & $\mathrm{H}$ & 13.2849 & 7.9679 & 5.0114 \\
$\mathrm{C}$ & 8.3578 & 15.8511 & 10.6785 & $\mathrm{H}$ & 10.0149 & 12.2349 & 5.9978 \\
$\mathrm{C}$ & 9.498 & 15.412 & 7.122 & $\mathrm{H}$ & 10.1441 & 14.6019 & 5.2388 \\
$\mathrm{C}$ & 8.1996 & 14.5297 & 11.0768 & $\mathrm{H}$ & 8.1162 & 16.654 & 11.3706 \\
\hline
\end{tabular}

Cartesian coordinate, $\mathbf{3}, \mathrm{S}$, Gas State

Cartesian coordinate, 5, S1, Gas State

\begin{tabular}{|c|c|c|c|c|c|c|c|}
\hline & $\mathrm{x}$ & $\mathrm{y}$ & $\mathrm{z}$ & & $\mathrm{x}$ & $\mathrm{y}$ & z \\
\hline $\mathrm{H}$ & 7.8319 & 14.2678 & 12.0633 & $\mathrm{Cu}$ & 7.2472 & 7.2666 & 6.2853 \\
\hline $\mathrm{H}$ & 7.2489 & 9.7943 & 10.4654 & $\mathrm{O}$ & 7.475447 & 4.59525 & 0.394993 \\
\hline $\mathrm{H}$ & 8.1456 & 9.2051 & 9.0284 & $\mathrm{O}$ & 6.664122 & 8.794802 & 1.966361 \\
\hline $\mathrm{H}$ & 9.3513 & 9.7251 & 11.7894 & $\mathrm{O}$ & 5.395589 & 1.910942 & 9.331358 \\
\hline $\mathrm{H}$ & 10.2336 & 9.1027 & 10.3827 & $\mathrm{O}$ & 4.12152 & 6.299698 & 9.33847 \\
\hline $\mathrm{H}$ & 8.8494 & 17.204 & 9.0697 & $\mathrm{~N}$ & 6.8508 & 4.386 & 6.324 \\
\hline $\mathrm{H}$ & 8.1932 & 7.9082 & 13.2108 & $\mathrm{~N}$ & 4.752105 & 4.111538 & 9.342331 \\
\hline $\mathrm{H}$ & 6.9298 & 7.9162 & 11.9715 & $\mathrm{~N}$ & 7.7678 & 5.0633 & 4.4591 \\
\hline $\mathrm{H}$ & 14.1668 & -0.7662 & 6.0027 & $\mathrm{~N}$ & 7.145955 & 6.715116 & 1.182959 \\
\hline $\mathrm{H}$ & 11.5187 & 0.9439 & 11.9176 & $\mathrm{C}$ & 7.912276 & 6.37389 & -1.1353 \\
\hline $\mathrm{H}$ & 13.5849 & 4.176 & 9.1752 & $\mathrm{C}$ & 7.497479 & 5.801772 & 0.17136 \\
\hline $\mathrm{H}$ & 14.5164 & 3.6757 & 7.734 & $\mathrm{C}$ & 4.359813 & 5.275626 & 11.4743 \\
\hline $\mathrm{H}$ & 11.0842 & -1.4615 & 12.4473 & $\mathrm{C}$ & 4.983989 & 2.887475 & 11.46608 \\
\hline $\mathrm{H}$ & 11.4958 & 4.24 & 7.8223 & $\mathrm{C}$ & 7.2953 & 5.4705 & 5.631 \\
\hline $\mathrm{H}$ & 12.3903 & 3.7148 & 6.3937 & $\mathrm{C}$ & 7.280673 & 8.626744 & -0.33986 \\
\hline $\mathrm{H}$ & 11.7029 & -3.2149 & 10.8262 & $\mathrm{C}$ & 4.642878 & 4.06766 & 12.18892 \\
\hline $\mathrm{H}$ & 13.7814 & -3.1895 & 6.503 & $\mathrm{C}$ & 6.991642 & 8.104328 & 1.000298 \\
\hline $\mathrm{H}$ & 14.6973 & 5.598 & 6.1152 & $\mathrm{C}$ & 4.404094 & 5.303159 & 10.00786 \\
\hline $\mathrm{H}$ & 13.3343 & 5.5783 & 4.9842 & $\mathrm{C}$ & 4.564042 & 4.049486 & 13.61102 \\
\hline $\mathrm{H}$ & 12.7344 & -3.8582 & 8.6376 & $\mathrm{C}$ & 7.784833 & 7.772804 & -1.35899 \\
\hline $\mathrm{H}$ & 9.6028 & -3.183 & 7.08 & $\mathrm{C}$ & 8.401656 & 5.561623 & -2.14291 \\
\hline $\mathrm{H}$ & 9.5475 & 16.4448 & 6.7849 & $\mathrm{H}$ & 8.462436 & 4.645472 & -1.99502 \\
\hline $\mathrm{H}$ & 12.5225 & 17.3449 & 8.7904 & $\mathrm{C}$ & 5.249506 & 1.713384 & 12.18037 \\
\hline $\mathrm{N}$ & 8.3735 & 2.2388 & 9.6561 & $\mathrm{H}$ & 5.477829 & 0.935295 & 11.72508 \\
\hline $\mathrm{N}$ & 8.8669 & 5.5968 & 11.2462 & $\mathrm{C}$ & 6.878237 & 6.140122 & 2.540432 \\
\hline $\mathrm{N}$ & 13.1171 & 11.2341 & 8.5785 & $\mathrm{H}$ & 6.431216 & 5.285197 & 2.439731 \\
\hline $\mathrm{N}$ & 12.7449 & 7.8267 & 7.0449 & $\mathrm{H}$ & 6.280822 & 6.732467 & 3.023203 \\
\hline $\mathrm{N}$ & 8.5639 & 11.1343 & 9.6145 & $\mathrm{C}$ & 5.070961 & 2.885639 & 9.992347 \\
\hline $\mathrm{N}$ & 8.908 & 7.7689 & 11.2333 & $\mathrm{C}$ & 3.963171 & 6.416001 & 13.59182 \\
\hline $\mathrm{N}$ & 13.2396 & 2.2507 & 8.5153 & $\mathrm{H}$ & 3.736424 & 7.194382 & 14.04739 \\
\hline $\mathrm{N}$ & 12.7799 & 5.6559 & 7.0158 & $\mathrm{C}$ & 6.4306 & 4.3858 & 7.7175 \\
\hline $\mathrm{O}$ & 7.3794 & 1.6346 & 11.6035 & $\mathrm{H}$ & 6.6839 & 5.2291 & 8.1243 \\
\hline $\mathrm{O}$ & 9.54 & 2.8741 & 7.81 & $\mathrm{H}$ & 6.8971 & 3.6775 & 8.1883 \\
\hline $\mathrm{O}$ & 13.8997 & 11.8762 & 6.5429 & $\mathrm{C}$ & 4.217682 & 5.255188 & 14.29234 \\
\hline $\mathrm{O}$ & 12.2927 & 10.6516 & 10.624 & $\mathrm{H}$ & 4.162934 & 5.258082 & 15.22073 \\
\hline $\mathrm{O}$ & 7.8379 & 11.7625 & 11.6795 & $\mathrm{C}$ & 7.6714 & 3.6008 & 4.2452 \\
\hline $\mathrm{O}$ & 9.5241 & 10.3915 & 7.6763 & $\mathrm{H}$ & 8.5394 & 3.2135 & 4.0516 \\
\hline $\mathrm{O}$ & 14.0962 & 1.6944 & 6.4915 & $\mathrm{H}$ & 7.0581 & 3.3882 & 3.5244 \\
\hline
\end{tabular}




\begin{tabular}{ccccccc}
$\mathrm{O} 12.2718$ & 2.8474 & 10.4878 & $\mathrm{C}$ & 4.9122 & 4.184 & 7.8841 \\
& & $\mathrm{H}$ & 4.4377 & 4.9297 & 7.4844 \\
& & $\mathrm{H}$ & 4.6386 & 3.3723 & 7.429 \\
& & $\mathrm{C}$ & 5.172689 & 1.706989 & 13.5722 \\
& & $\mathrm{H}$ & 5.342669 & 0.914212 & 14.02774 \\
& & $\mathrm{C}$ & 4.855652 & 2.826987 & 14.29092 \\
\hline
\end{tabular}

\begin{tabular}{|c|c|c|c|c|c|c|c|}
\hline \multicolumn{8}{|c|}{ Cartesian coordinate, $\mathbf{5}, \mathrm{S} 1$, Gas State } \\
\hline & $\mathrm{x}$ & $\mathrm{y}$ & $\mathrm{z}$ & & $\mathrm{x}$ & $\mathrm{y}$ & $\mathrm{z}$ \\
\hline $\mathrm{H}$ & 4.829949 & 2.792861 & 15.21996 & $\mathrm{H}$ & 7.888838 & 9.203388 & 10.23956 \\
\hline $\mathrm{C}$ & 4.046468 & 6.421179 & 12.19642 & $\mathrm{H}$ & 8.090131 & 7.767192 & 9.644186 \\
\hline $\mathrm{H}$ & 3.887574 & 7.21445 & 11.73771 & $\mathrm{C}$ & 9.843762 & 11.85073 & 2.929587 \\
\hline $\mathrm{C}$ & 8.143972 & 8.315348 & -2.62993 & $\mathrm{C}$ & 12.08987 & 8.544302 & -0.33681 \\
\hline $\mathrm{C}$ & 7.099506 & 9.972883 & -0.62032 & $\mathrm{H}$ & 12.47393 & 7.798241 & -0.73773 \\
\hline $\mathrm{H}$ & 6.747994 & 10.53211 & 0.034333 & $\mathrm{C}$ & 7.960058 & 10.18086 & 4.865253 \\
\hline $\mathrm{C}$ & 8.804836 & 6.075441 & -3.36983 & $\mathrm{H}$ & 7.750166 & 9.327355 & 4.454772 \\
\hline $\mathrm{H}$ & 9.162959 & 5.507897 & -4.01371 & $\mathrm{H}$ & 7.470561 & 10.86821 & 4.386856 \\
\hline $\mathrm{C}$ & 8.675979 & 7.431053 & -3.63937 & $\mathrm{C}$ & 11.98264 & 9.726999 & -1.03875 \\
\hline $\mathrm{H}$ & 8.931467 & 7.768428 & -4.46749 & $\mathrm{H}$ & 12.29452 & 9.771203 & -1.9138 \\
\hline $\mathrm{C}$ & 8.1579 & 5.9423 & 3.3611 & $\mathrm{C}$ & 6.631121 & 10.90971 & 8.317133 \\
\hline $\mathrm{H}$ & 8.8609 & 5.5266 & 2.8377 & $\mathrm{H}$ & 5.74409 & 11.25883 & 8.496788 \\
\hline $\mathrm{H}$ & 8.4941 & 6.7817 & 3.7121 & $\mathrm{H}$ & 7.223038 & 11.1487 & 9.04751 \\
\hline $\mathrm{C}$ & 7.951975 & 9.71499 & -2.85134 & $\mathrm{C}$ & 9.473047 & 10.45907 & 4.94973 \\
\hline $\mathrm{H}$ & 8.181614 & 10.08747 & -3.67194 & $\mathrm{H}$ & 9.915021 & 9.726349 & 5.406548 \\
\hline $\mathrm{C}$ & 7.436516 & 10.50644 & -1.87259 & $\mathrm{H}$ & 9.628136 & 11.27034 & 5.458435 \\
\hline $\mathrm{H}$ & 7.305948 & 11.41263 & -2.03597 & $\mathrm{C}$ & 10.68614 & 13.19653 & -0.49006 \\
\hline $\mathrm{C}$ & 7.1336 & 3.1201 & 5.597 & $\mathrm{H}$ & 10.6102 & 14.00329 & -0.94642 \\
\hline $\mathrm{H}$ & 6.3263 & 2.5944 & 5.4845 & $\mathrm{C}$ & 11.24754 & 12.12574 & -1.13013 \\
\hline $\mathrm{H}$ & 7.795 & 2.5882 & 6.0666 & $\mathrm{H}$ & 11.52987 & 12.2059 & -2.01263 \\
\hline $\mathrm{O}$ & 6.748747 & 9.851454 & 12.23553 & $\mathrm{C}$ & 11.62063 & 8.468314 & 0.977776 \\
\hline $\mathrm{O}$ & 7.702741 & 5.689421 & 10.64434 & $\mathrm{H}$ & 11.68507 & 7.660477 & 1.434048 \\
\hline $\mathrm{O}$ & 9.298559 & 12.7789 & 3.506972 & $\mathrm{C}$ & 6.042097 & 6.079803 & 15.18705 \\
\hline $\mathrm{O}$ & 10.74439 & 8.4482 & 3.701741 & $\mathrm{C}$ & 7.194347 & 4.469445 & 13.19811 \\
\hline $\mathrm{N}$ & 7.518091 & 10.16175 & 6.251901 & $\mathrm{H}$ & 7.581349 & 3.925817 & 12.55037 \\
\hline $\mathrm{N}$ & 10.03009 & 10.608 & 3.598862 & $\mathrm{C}$ & 5.30904 & 8.296465 & 15.92895 \\
\hline $\mathrm{N}$ & 6.602084 & 9.444573 & 8.102237 & $\mathrm{H}$ & 4.915578 & 8.848434 & 16.56574 \\
\hline $\mathrm{N}$ & 7.15009 & 7.748855 & 11.43463 & $\mathrm{C}$ & 5.454916 & 6.940242 & 16.18648 \\
\hline $\mathrm{C}$ & 6.290643 & 8.04477 & 13.72623 & $\mathrm{H}$ & 5.175366 & 6.587142 & 17.00015 \\
\hline $\mathrm{C}$ & 6.742616 & 8.641846 & 12.44325 & $\mathrm{C}$ & 6.23329 & 8.54902 & 9.194207 \\
\hline $\mathrm{C}$ & 11.06052 & 9.564487 & 1.623047 & $\mathrm{H}$ & 5.504613 & 8.933392 & 9.706231 \\
\hline $\mathrm{C}$ & 10.33877 & 11.92356 & 1.540648 & $\mathrm{H}$ & 5.939526 & 7.695877 & 8.838201 \\
\hline $\mathrm{C}$ & 7.110348 & 9.058664 & 6.93813 & $\mathrm{C}$ & 6.253766 & 4.681358 & 15.39826 \\
\hline $\mathrm{C}$ & 6.996829 & 5.815243 & 12.92751 & $\mathrm{H}$ & 6.001029 & 4.293766 & 16.20496 \\
\hline $\mathrm{C}$ & 10.92742 & 10.79382 & 0.901328 & $\mathrm{C}$ & 6.821376 & 3.913084 & 14.4301 \\
\hline $\mathrm{C}$ & 7.325386 & 6.360671 & 11.60576 & $\mathrm{H}$ & 6.96393 & 3.007501 & 14.58706 \\
\hline $\mathrm{C}$ & 10.60998 & 9.464251 & 3.015935 & $\mathrm{C}$ & 7.168854 & 11.41387 & 6.973879 \\
\hline
\end{tabular}




\begin{tabular}{llllllll}
$\mathrm{C}$ & 11.39923 & 10.88391 & -0.43974 & $\mathrm{H}$ & 7.950572 & 11.97433 & 7.099006 \\
$\mathrm{C}$ & 6.437697 & 6.645826 & 13.93736 & $\mathrm{H}$ & 6.492429 & 11.91669 & 6.493649 \\
$\mathrm{C}$ & 5.747567 & 8.834061 & 14.72456 & & & & \\
$\mathrm{H}$ & 5.673976 & 9.750694 & 14.58575 & & & & \\
$\mathrm{C}$ & 10.22416 & 13.11975 & 0.822889 & & & & \\
$\mathrm{H}$ & 9.838599 & 13.86547 & 1.223034 & & & & \\
$\mathrm{C}$ & 7.456803 & 8.346986 & 10.09538 & & & & \\
\hline
\end{tabular}

\begin{tabular}{|c|c|c|c|c|c|c|c|}
\hline \multicolumn{8}{|c|}{ Cartesian coordinate, $\mathbf{6}, \mathrm{S} 1$, Gas State } \\
\hline & $\mathrm{x}$ & $\mathrm{y}$ & $\mathrm{z}$ & & $\mathrm{x}$ & $\mathrm{y}$ & $\mathrm{z}$ \\
\hline $\mathrm{Ag}$ & 10.6071 & 6.616 & 8.6216 & $\mathrm{C}$ & 7.9643 & 9.7446 & 9.6365 \\
\hline $\mathrm{C}$ & 8.8894 & 0.3004 & 8.0872 & $\mathrm{C}$ & 9.067 & 9.0829 & 10.467 \\
\hline $\mathrm{C}$ & 8.6806 & -0.6322 & 9.129 & $\mathrm{C}$ & 8.5555 & 16.194 & 9.7895 \\
\hline $\mathrm{C}$ & 9.4087 & -0.114 & 6.8524 & $\mathrm{C}$ & 7.726 & 7.4175 & 11.8227 \\
\hline $\mathrm{C}$ & 8.0394 & -0.2206 & 10.3097 & $\mathrm{C}$ & 13.6775 & 1.385 & 7.3361 \\
\hline $\mathrm{C}$ & 7.7192 & 1.2108 & 10.5304 & $\mathrm{C}$ & 12.8242 & -0.0098 & 9.2124 \\
\hline $\mathrm{C}$ & 8.5863 & 1.7362 & 8.2851 & $\mathrm{C}$ & 13.4863 & 0.0913 & 7.9648 \\
\hline $\mathrm{C}$ & 9.087 & -1.9781 & 8.979 & $\mathrm{C}$ & 12.3456 & 1.1513 & 9.8746 \\
\hline $\mathrm{C}$ & 9.8317 & -1.4098 & 6.6788 & $\mathrm{C}$ & 12.4914 & 2.4464 & 9.2595 \\
\hline $\mathrm{C}$ & 8.071 & -2.4921 & 11.1111 & $\mathrm{C}$ & 13.9495 & -1.0648 & 7.3145 \\
\hline $\mathrm{C}$ & 9.8205 & -2.3214 & 7.7788 & $\mathrm{C}$ & 11.7028 & 1.0451 & 11.1276 \\
\hline $\mathrm{C}$ & 13.6268 & 12.0945 & 7.4229 & $\mathrm{C}$ & 13.418 & 3.8025 & 7.4808 \\
\hline $\mathrm{C}$ & 12.9833 & 13.5619 & 9.3475 & $\mathrm{C}$ & 12.5963 & -1.2993 & 9.8035 \\
\hline $\mathrm{C}$ & 7.7334 & -1.1493 & 11.2932 & $\mathrm{C}$ & 11.5105 & -0.1985 & 11.7012 \\
\hline $\mathrm{C}$ & 7.7869 & 3.5223 & 9.7455 & $\mathrm{C}$ & 12.2063 & 4.2725 & 6.6717 \\
\hline $\mathrm{C}$ & 13.5566 & 13.4083 & 8.0647 & $\mathrm{C}$ & 11.9266 & -1.3631 & 11.0508 \\
\hline $\mathrm{C}$ & 8.9547 & 4.1654 & 10.5025 & $\mathrm{C}$ & 13.043 & -2.4413 & 9.0904 \\
\hline $\mathrm{C}$ & 12.4386 & 12.4426 & 10.0186 & $\mathrm{C}$ & 13.7234 & -2.3119 & 7.8772 \\
\hline $\mathrm{C}$ & 12.5096 & 11.1041 & 9.404 & $\mathrm{C}$ & 13.3037 & 6.0379 & 5.2191 \\
\hline $\mathrm{C}$ & 8.7584 & -2.9039 & 9.9739 & $\mathrm{H}$ & 9.5172 & 0.6236 & 6.0648 \\
\hline $\mathrm{C}$ & 14.0699 & 14.5202 & 7.4175 & $\mathrm{H}$ & 10.3057 & -1.7137 & 5.7513 \\
\hline $\mathrm{C}$ & 7.8672 & 5.9056 & 11.9804 & $\mathrm{H}$ & 7.8079 & -3.2212 & 11.8703 \\
\hline $\mathrm{C}$ & 9.2184 & 6.6185 & 10.2151 & $\mathrm{H}$ & 7.232 & -0.8092 & 12.1935 \\
\hline $\mathrm{C}$ & 11.8463 & 12.5808 & 11.2589 & $\mathrm{H}$ & 6.8618 & 3.5962 & 10.3208 \\
\hline $\mathrm{C}$ & 13.3382 & 9.6705 & 7.6323 & $\mathrm{H}$ & 7.6546 & 4.0028 & 8.7756 \\
\hline $\mathrm{C}$ & 12.9363 & 14.8379 & 9.9749 & $\mathrm{H}$ & 9.1157 & 3.6311 & 11.4462 \\
\hline $\mathrm{C}$ & 11.7657 & 13.8434 & 11.8628 & $\mathrm{H}$ & 9.8875 & 4.0993 & 9.9343 \\
\hline $\mathrm{C}$ & 12.1265 & 9.1721 & 6.8399 & $\mathrm{H}$ & 9.0169 & -3.9511 & 9.8503 \\
\hline $\mathrm{C}$ & 12.3 & 14.9471 & 11.2313 & $\mathrm{H}$ & 14.5046 & 14.3877 & 6.4316 \\
\hline $\mathrm{C}$ & 13.5296 & 15.9577 & 9.2988 & $\mathrm{H}$ & 8.4183 & 5.6247 & 12.8877 \\
\hline $\mathrm{C}$ & 14.0619 & 15.7752 & 8.0237 & $\mathrm{H}$ & 6.9063 & 5.3856 & 11.9906 \\
\hline $\mathrm{C}$ & 11.889 & 6.7052 & 6.9451 & $\mathrm{H}$ & 11.4471 & 11.697 & 11.7457 \\
\hline $\mathrm{C}$ & 13.1025 & 7.5512 & 5.1425 & $\mathrm{H}$ & 13.517 & 8.9944 & 8.471 \\
\hline $\mathrm{C}$ & 9.1107 & 12.9518 & 8.0158 & $\mathrm{H}$ & 14.2226 & 9.711 & 6.9938 \\
\hline $\mathrm{C}$ & 8.7157 & 13.9015 & 8.9852 & $\mathrm{H}$ & 11.2773 & 13.952 & 12.8255 \\
\hline $\mathrm{C}$ & 9.7147 & 13.3895 & 6.8478 & $\mathrm{H}$ & 11.2274 & 9.1881 & 7.4601 \\
\hline
\end{tabular}




\begin{tabular}{cccccccc}
$\mathrm{C}$ & 8.1139 & 13.4728 & 10.1911 & $\mathrm{H}$ & 11.9436 & 9.8233 & 5.9782 \\
$\mathrm{C}$ & 7.8763 & 12.037 & 10.4326 & $\mathrm{H}$ & 12.1953 & 15.919 & 11.7056 \\
$\mathrm{C}$ & 8.8959 & 11.5216 & 8.2294 & $\mathrm{H}$ & 14.5002 & 16.6252 & 7.507 \\
$\mathrm{C}$ & 8.9281 & 15.2917 & 8.7687 & $\mathrm{H}$ & 14.042 & 8.1085 & 5.1143 \\
$\mathrm{C}$ & 9.9136 & 14.7453 & 6.6026 & $\mathrm{H}$ & 12.4982 & 7.8526 & 4.2769 \\
$\mathrm{C}$ & 7.9734 & 15.753 & 10.9606 & $\mathrm{H}$ & 10.0161 & 12.6494 & 6.1132 \\
$\mathrm{C}$ & 9.5191 & 15.7115 & 7.5273 & $\mathrm{H}$ & 10.3717 & 15.0613 & 5.6689 \\
$\mathrm{C}$ & 7.7446 & 14.3844 & 11.1613 & $\mathrm{H}$ & 7.6949 & 16.4666 & 11.7297 \\
\hline
\end{tabular}

\begin{tabular}{|c|c|c|c|c|c|c|c|}
\hline \multicolumn{4}{|c|}{ Cartesian coordinate, $\mathbf{6}, \mathrm{S} 1$, Gas State } & \multicolumn{4}{|c|}{ Cartesian coordinate, $\mathrm{Ag}^{\mathrm{I}}-\mathrm{NHC}_{2}-\mathrm{NH}, \mathrm{S} 1$, Gas State } \\
\hline & $\mathrm{x}$ & $\mathrm{y}$ & $\mathrm{z}$ & & $\mathrm{x}$ & $\mathrm{y}$ & $\mathrm{z}$ \\
\hline $\mathrm{H}$ & 7.2852 & 14.0149 & 12.073 & $\mathrm{Ag}$ & 10.7122 & 6.6625 & 9.064 \\
\hline $\mathrm{H}$ & 7.0052 & 9.6867 & 10.1565 & $\mathrm{C}$ & 8.2652 & 0.5336 & 7.8326 \\
\hline $\mathrm{H}$ & 7.8898 & 9.2657 & 8.6591 & $\mathrm{C}$ & 7.3861 & -0.2351 & 8.6242 \\
\hline $\mathrm{H}$ & 9.2062 & 9.6472 & 11.3953 & $\mathrm{C}$ & 8.8664 & -0.0845 & 6.7046 \\
\hline $\mathrm{H}$ & 10.0162 & 9.0948 & 9.928 & $\mathrm{C}$ & 6.7994 & 0.32 & 9.7909 \\
\hline $\mathrm{H}$ & 8.7499 & 17.257 & 9.6757 & $\mathrm{C}$ & 7.0737 & 1.7108 & 10.1583 \\
\hline $\mathrm{H}$ & 7.9242 & 7.9719 & 12.7444 & $\mathrm{C}$ & 8.5672 & 1.8962 & 8.1601 \\
\hline $\mathrm{H}$ & 6.7324 & 7.7064 & 11.4584 & $\mathrm{C}$ & 7.0756 & -1.5909 & 8.2796 \\
\hline $\mathrm{H}$ & 14.4808 & -0.9506 & 6.376 & $\mathrm{C}$ & 8.5777 & -1.3629 & 6.3305 \\
\hline $\mathrm{H}$ & 11.3936 & 1.9583 & 11.6236 & $\mathrm{C}$ & 5.6905 & -1.7597 & 10.2892 \\
\hline $\mathrm{H}$ & 13.5899 & 4.5032 & 8.3013 & $\mathrm{C}$ & 7.6323 & -2.1946 & 7.0409 \\
\hline $\mathrm{H}$ & 14.3034 & 3.7266 & 6.847 & $\mathrm{C}$ & 14.1093 & 11.9478 & 7.8016 \\
\hline $\mathrm{H}$ & 11.0322 & -0.2762 & 12.6738 & $\mathrm{C}$ & 13.4663 & 13.8807 & 9.2195 \\
\hline $\mathrm{H}$ & 11.3042 & 4.1903 & 7.2827 & $\mathrm{C}$ & 5.9656 & -0.4348 & 10.6076 \\
\hline $\mathrm{H}$ & 12.0828 & 3.6432 & 5.7837 & $\mathrm{C}$ & 8.1261 & 3.8155 & 9.6433 \\
\hline $\mathrm{H}$ & 11.731 & -2.3211 & 11.5231 & $\mathrm{C}$ & 14.2135 & 13.3331 & 8.1462 \\
\hline $\mathrm{H}$ & 14.0879 & -3.2069 & 7.3729 & $\mathrm{C}$ & 9.2147 & 3.9775 & 10.7083 \\
\hline $\mathrm{H}$ & 14.3204 & 5.7688 & 5.5315 & $\mathrm{C}$ & 12.5913 & 13.0628 & 9.975 \\
\hline $\mathrm{H}$ & 13.0882 & 5.524 & 4.2779 & $\mathrm{C}$ & 12.4832 & 11.631 & 9.6792 \\
\hline $\mathrm{H}$ & 10.9978 & -3.8174 & 8.5642 & $\mathrm{C}$ & 6.2474 & -2.3136 & 9.1371 \\
\hline $\mathrm{H}$ & 11.0614 & -3.593 & 6.8687 & $\mathrm{C}$ & 15.0535 & 14.1977 & 7.4088 \\
\hline $\mathrm{H}$ & 13.2892 & -4.4476 & 9.183 & $\mathrm{C}$ & 8.3857 & 5.4737 & 12.5673 \\
\hline $\mathrm{H}$ & 12.7037 & -3.8087 & 10.5625 & $\mathrm{C}$ & 9.5783 & 6.4367 & 10.8116 \\
\hline $\mathrm{H}$ & 13.5557 & 17.2403 & 10.8932 & $\mathrm{C}$ & 11.8263 & 13.5979 & 11.0069 \\
\hline $\mathrm{H}$ & 14.1087 & 17.8978 & 9.4451 & $\mathrm{C}$ & 13.2219 & 9.737 & 8.3532 \\
\hline $\mathrm{H}$ & 9.984 & 17.2841 & 6.3277 & $\mathrm{C}$ & 13.5707 & 15.2612 & 9.5513 \\
\hline $\mathrm{H}$ & 9.1337 & 17.7161 & 7.7201 & $\mathrm{C}$ & 11.9283 & 14.9499 & 11.3213 \\
\hline $\mathrm{N}$ & 8.0298 & 2.1009 & 9.5028 & $\mathrm{C}$ & 12.1314 & 9.3422 & 7.3513 \\
\hline $\mathrm{N}$ & 8.6631 & 5.5486 & 10.8003 & $\mathrm{C}$ & 12.7966 & 15.7631 & 10.5983 \\
\hline $\mathrm{N}$ & 13.1499 & 11.0067 & 8.1763 & $\mathrm{C}$ & 14.4704 & 16.1561 & 8.7942 \\
\hline $\mathrm{N}$ & 12.3546 & 7.821 & 6.3752 & $\mathrm{C}$ & 15.1845 & 15.5271 & 7.7057 \\
\hline $\mathrm{N}$ & 8.245 & 11.1564 & 9.4163 & $\mathrm{C}$ & 11.9638 & 6.8565 & 7.3958 \\
\hline $\mathrm{N}$ & 8.7456 & 7.7151 & 10.8124 & $\mathrm{C}$ & 13.2757 & 7.7965 & 5.7131 \\
\hline $\mathrm{N}$ & 13.1951 & 2.4908 & 8.0527 & $\mathrm{C}$ & 8.4701 & 12.0484 & 8.0633 \\
\hline $\mathrm{N}$ & 12.3383 & 5.6536 & 6.254 & $\mathrm{C}$ & 8.1222 & 13.1696 & 8.8489 \\
\hline
\end{tabular}




\begin{tabular}{cccccccc}
$\mathrm{N}$ & 10.5223 & -3.4436 & 7.7139 & $\mathrm{C}$ & 8.777 & 12.2517 & 6.6916 \\
$\mathrm{~N}$ & 12.6496 & -3.7452 & 9.5483 & $\mathrm{C}$ & 7.8032 & 13.009 & 10.2221 \\
$\mathrm{~N}$ & 13.5319 & 17.1963 & 9.8858 & $\mathrm{C}$ & 7.7851 & 11.6737 & 10.8229 \\
$\mathrm{~N}$ & 9.7313 & 17.0387 & 7.2732 & $\mathrm{C}$ & 8.517 & 10.7374 & 8.6259 \\
$\mathrm{O}$ & 7.2196 & 1.605 & 11.5656 & $\mathrm{C}$ & 8.0913 & 14.4838 & 8.2821 \\
$\mathrm{O}$ & 8.8269 & 2.5518 & 7.415 & $\mathrm{C}$ & 8.729 & 13.4802 & 6.1082 \\
$\mathrm{O}$ & 14.0814 & 11.9038 & 6.3055 & $\mathrm{C}$ & 7.4779 & 15.3816 & 10.4736 \\
$\mathrm{O}$ & 12.0483 & 10.1058 & 9.9424 & $\mathrm{C}$ & 8.3876 & 14.6828 & 6.8377 \\
$\mathrm{O}$ & 7.3776 & 11.6102 & 11.4627 & $\mathrm{C}$ & 7.4884 & 14.1042 & 11.0196 \\
\hline
\end{tabular}

Cartesian coordinate, $\mathrm{Ag}^{\mathrm{I}}-\mathrm{NHC}_{2}-\mathrm{NH}, \mathrm{S} 1$, Gas State

\begin{tabular}{|c|c|c|c|c|c|c|c|}
\hline & $\mathrm{x}$ & $\mathrm{y}$ & z & & $\mathrm{x}$ & $\mathrm{y}$ & z \\
\hline $\mathrm{C}$ & 8.0091 & 9.2858 & 10.5271 & $\mathrm{H}$ & 7.2566 & 13.9211 & 12.0651 \\
\hline $\mathrm{C}$ & 9.2877 & 8.8498 & 11.2499 & $\mathrm{H}$ & 7.1617 & 9.2738 & 11.2185 \\
\hline $\mathrm{C}$ & 7.7749 & 15.5506 & 9.1217 & $\mathrm{H}$ & 7.8332 & 8.6048 & 9.692 \\
\hline $\mathrm{C}$ & 8.4097 & 6.9971 & 12.7515 & $\mathrm{H}$ & 9.4363 & 9.4497 & 12.1529 \\
\hline $\mathrm{C}$ & 14.5273 & 1.9257 & 7.7117 & $\mathrm{H}$ & 10.1549 & 9.0069 & 10.604 \\
\hline $\mathrm{C}$ & 13.9322 & 0.087 & 9.2932 & $\mathrm{H}$ & 7.7626 & 16.5593 & 8.7121 \\
\hline $\mathrm{C}$ & 14.6541 & 0.5426 & 8.1631 & $\mathrm{H}$ & 8.8828 & 7.304 & 13.6923 \\
\hline $\mathrm{C}$ & 13.0861 & 0.9733 & 10.0142 & $\mathrm{H}$ & 7.4101 & 7.4445 & 12.7189 \\
\hline $\mathrm{C}$ & 12.9722 & 2.3534 & 9.6182 & $\mathrm{H}$ & 16.008 & 0.0446 & 6.593 \\
\hline $\mathrm{C}$ & 15.4889 & -0.3275 & 7.4703 & $\mathrm{H}$ & 11.718 & 1.1997 & 11.6518 \\
\hline $\mathrm{C}$ & 12.3624 & 0.5014 & 11.1282 & $\mathrm{H}$ & 13.4937 & 4.7324 & 9.0064 \\
\hline $\mathrm{C}$ & 13.6302 & 4.1424 & 8.0975 & $\mathrm{H}$ & 14.5799 & 4.3914 & 7.6175 \\
\hline $\mathrm{C}$ & 14.0609 & -1.2717 & 9.7178 & $\mathrm{H}$ & 11.9102 & -1.1772 & 12.3819 \\
\hline $\mathrm{C}$ & 12.4827 & -0.8233 & 11.5262 & $\mathrm{H}$ & 11.5068 & 4.2121 & 7.6006 \\
\hline $\mathrm{C}$ & 12.4734 & 4.4381 & 7.1387 & $\mathrm{H}$ & 12.559 & 3.8134 & 6.2438 \\
\hline $\mathrm{C}$ & 13.3176 & -1.7101 & 10.8536 & $\mathrm{H}$ & 13.3788 & -2.744 & 11.1701 \\
\hline $\mathrm{C}$ & 14.9238 & -2.1262 & 8.9943 & $\mathrm{H}$ & 16.2775 & -2.3342 & 7.3539 \\
\hline $\mathrm{C}$ & 15.6332 & -1.6487 & 7.9004 & $\mathrm{H}$ & 14.462 & 5.9494 & 5.9246 \\
\hline $\mathrm{C}$ & 13.4412 & 6.2725 & 5.6874 & $\mathrm{H}$ & 13.1665 & 5.8311 & 4.7226 \\
\hline $\mathrm{H}$ & 9.5798 & 0.5097 & 6.1383 & $\mathrm{H}$ & 6.6494 & -3.8302 & 7.1576 \\
\hline $\mathrm{H}$ & 9.0402 & -1.8098 & 5.4552 & $\mathrm{H}$ & 15.8629 & -3.4393 & 10.0637 \\
\hline $\mathrm{H}$ & 5.0539 & -2.3622 & 10.9336 & $\mathrm{H}$ & 15.2055 & 17.8706 & 8.4898 \\
\hline $\mathrm{H}$ & 5.5538 & 0.0426 & 11.4923 & $\mathrm{H}$ & 8.1185 & 16.576 & 6.8272 \\
\hline $\mathrm{H}$ & 7.1829 & 4.2194 & 10.0221 & $\mathrm{~N}$ & 7.9016 & 2.4221 & 9.3072 \\
\hline $\mathrm{H}$ & 8.4148 & 4.333 & 8.7267 & $\mathrm{~N}$ & 9.1485 & 5.2886 & 11.3319 \\
\hline $\mathrm{H}$ & 9.0428 & 3.2408 & 11.4978 & $\mathrm{~N}$ & 13.2578 & 11.1599 & 8.6246 \\
\hline $\mathrm{H}$ & 10.2153 & 3.804 & 10.3009 & $\mathrm{~N}$ & 12.3711 & 8.0008 & 6.8457 \\
\hline $\mathrm{H}$ & 6.0304 & -3.3546 & 8.9037 & $\mathrm{~N}$ & 8.0999 & 10.6214 & 9.9792 \\
\hline $\mathrm{H}$ & 15.6022 & 13.7589 & 6.5778 & $\mathrm{~N}$ & 9.2154 & 7.4455 & 11.6143 \\
\hline $\mathrm{H}$ & 8.8779 & 4.9412 & 13.39 & $\mathrm{~N}$ & 13.7084 & 2.7476 & 8.4792 \\
\hline $\mathrm{H}$ & 7.3784 & 5.0567 & 12.4556 & $\mathrm{~N}$ & 12.5073 & 5.832 & 6.7265 \\
\hline $\mathrm{H}$ & 11.1524 & 12.9359 & 11.5426 & $\mathrm{~N}$ & 7.3423 & -3.3727 & 6.5596 \\
\hline $\mathrm{H}$ & 13.0513 & 9.216 & 9.2983 & $\mathrm{~N}$ & 15.0736 & -3.5038 & 9.3983 \\
\hline $\mathrm{H}$ & 14.2008 & 9.4697 & 7.9491 & $\mathrm{~N}$ & 14.5586 & 17.4134 & 9.139 \\
\hline
\end{tabular}




\begin{tabular}{cccccccc}
$\mathrm{H}$ & 11.3208 & 15.3703 & 12.1192 & $\mathrm{~N}$ & 8.3593 & 15.8131 & 6.1904 \\
$\mathrm{H}$ & 11.1319 & 9.3909 & 7.794 & $\mathrm{O}$ & 6.5943 & 2.2512 & 11.1616 \\
$\mathrm{H}$ & 12.1543 & 10.0308 & 6.5001 & $\mathrm{O}$ & 9.3275 & 2.6391 & 7.5326 \\
$\mathrm{H}$ & 12.8972 & 16.8224 & 10.82 & $\mathrm{O}$ & 14.7005 & 11.3871 & 6.8692 \\
$\mathrm{H}$ & 15.8434 & 16.1575 & 7.1076 & $\mathrm{O}$ & 11.7737 & 10.8578 & 10.3251 \\
$\mathrm{H}$ & 14.2121 & 8.3427 & 5.8681 & $\mathrm{O}$ & 7.4905 & 11.4593 & 12.003 \\
$\mathrm{H}$ & 12.812 & 8.1861 & 4.7981 & $\mathrm{O}$ & 8.8538 & 9.7038 & 8.0289 \\
$\mathrm{H}$ & 9.0491 & 11.3722 & 6.1132 & $\mathrm{O}$ & 15.1148 & 2.3789 & 6.7225 \\
$\mathrm{H}$ & 8.9653 & 13.6174 & 5.0568 & $\mathrm{O}$ & 12.2903 & 3.2047 & 10.2124 \\
$\mathrm{H}$ & 7.2443 & 16.2445 & 11.0924 & & & & \\
\hline
\end{tabular}

\begin{tabular}{cccccccc} 
Cartesian coordinate, $6, \mathrm{~S} 0$, Gas State & \multicolumn{7}{c}{} \\
\multicolumn{1}{c}{} & $\mathrm{x}$ & $\mathrm{y}$ & $\mathrm{z}$ & & $\mathrm{x}$ & $\mathrm{y}$ & $\mathrm{z}$ \\
\hline $\mathrm{Ag}$ & 9.7817 & 6.7776 & 9.3628 & $\mathrm{C}$ & 6.8052 & 9.4594 & 9.3658 \\
$\mathrm{C}$ & 9.5031 & 0.4642 & 7.3557 & $\mathrm{C}$ & 7.3058 & 8.7657 & 10.6372 \\
$\mathrm{C}$ & 8.8828 & -0.6312 & 7.9973 & $\mathrm{C}$ & 8.0733 & 15.7963 & 9.7771 \\
$\mathrm{C}$ & 10.4988 & 0.2237 & 6.4213 & $\mathrm{C}$ & 5.7329 & 6.8035 & 11.072 \\
$\mathrm{C}$ & 7.8772 & -0.4041 & 8.966 & $\mathrm{C}$ & 13.8152 & 2.1382 & 8.8193 \\
$\mathrm{C}$ & 7.4307 & 0.971 & 9.2683 & $\mathrm{C}$ & 13.0892 & -0.0633 & 9.753 \\
$\mathrm{C}$ & 9.0946 & 1.8389 & 7.6462 & $\mathrm{C}$ & 13.9837 & 0.6949 & 8.9638 \\
$\mathrm{C}$ & 9.2662 & -1.9663 & 7.6857 & $\mathrm{C}$ & 11.9792 & 0.5638 & 10.3675 \\
$\mathrm{C}$ & 10.8682 & -1.0741 & 6.0746 & $\mathrm{C}$ & 11.765 & 2.015 & 10.2132 \\
$\mathrm{C}$ & 7.6892 & -2.7766 & 9.3537 & $\mathrm{C}$ & 15.0443 & 0.0588 & 8.3389 \\
$\mathrm{C}$ & 10.2573 & -2.1789 & 6.666 & $\mathrm{C}$ & 11.0835 & -0.1667 & 11.1252 \\
$\mathrm{C}$ & 14.344 & 11.6688 & 8.4909 & $\mathrm{C}$ & 12.6024 & 4.1593 & 9.3906 \\
$\mathrm{C}$ & 14.0809 & 14.0996 & 9.0122 & $\mathrm{C}$ & 13.2882 & -1.4603 & 9.9343 \\
$\mathrm{C}$ & 7.286 & -1.4612 & 9.6304 & $\mathrm{C}$ & 11.2699 & -1.5455 & 11.2953 \\
$\mathrm{C}$ & 7.5301 & 3.3493 & 8.7917 & $\mathrm{C}$ & 12.0045 & 4.6174 & 8.053 \\
$\mathrm{C}$ & 14.7131 & 13.0679 & 8.2823 & $\mathrm{C}$ & 12.351 & -2.1756 & 10.7126 \\
$\mathrm{C}$ & 7.9386 & 3.9226 & 10.1546 & $\mathrm{C}$ & 14.4304 & -2.0772 & 9.3156 \\
$\mathrm{C}$ & 13.0705 & 13.7851 & 9.952 & $\mathrm{C}$ & 15.2656 & -1.3054 & 8.5073 \\
$\mathrm{C}$ & 12.6557 & 12.3838 & 10.1675 & $\mathrm{C}$ & 13.5937 & 6.4159 & 7.2626 \\
$\mathrm{C}$ & 8.6575 & -3.0215 & 8.4003 & $\mathrm{H}$ & 10.9748 & 1.0733 & 5.9421 \\
$\mathrm{C}$ & 15.6916 & 13.3922 & 7.3569 & $\mathrm{H}$ & 11.6306 & -1.2356 & 5.3168 \\
$\mathrm{C}$ & 5.9563 & 5.2893 & 10.962 & $\mathrm{H}$ & 7.2366 & -3.6031 & 9.8922 \\
$\mathrm{C}$ & 7.8839 & 6.4014 & 10.2403 & $\mathrm{H}$ & 6.5156 & -1.2442 & 10.3641 \\
$\mathrm{C}$ & 12.4397 & 14.7821 & 10.6687 & $\mathrm{H}$ & 6.4394 & 3.2971 & 8.722 \\
$\mathrm{C}$ & 12.9412 & 10.0238 & 9.6125 & $\mathrm{H}$ & 7.9171 & 3.9776 & 7.988 \\
$\mathrm{C}$ & 14.4486 & 15.4596 & 8.8056 & $\mathrm{H}$ & 7.6326 & 3.2326 & 10.9464 \\
$\mathrm{C}$ & 12.7852 & 16.1242 & 10.4543 & $\mathrm{H}$ & 9.0225 & 4.0383 & 10.2146 \\
$\mathrm{C}$ & 11.9891 & 9.5058 & 8.5177 & $\mathrm{H}$ & 8.9729 & -4.0466 & 8.2257 \\
$\mathrm{C}$ & 13.7617 & 16.4549 & 9.5358 & $\mathrm{H}$ & 16.1676 & 12.5866 & 6.8071 \\
$\mathrm{C}$ & 15.4891 & 15.758 & 7.8583 & $\mathrm{H}$ & 5.9204 & 4.7796 & 11.9311 \\
$\mathrm{C}$ & 16.0768 & 14.7146 & 7.1444 & $\mathrm{H}$ & 5.2347 & 4.8002 & 10.3011 \\
$\mathrm{C}$ & 11.6382 & 7.0379 & 8.3733 & $\mathrm{H}$ & 11.6677 & 14.4994 & 11.3778 \\
$\mathrm{C}$ & 13.4445 & 7.9293 & 7.2072 & $\mathrm{H}$ & 12.4677 & 9.9601 & 10.5928 \\
& & & & & & &
\end{tabular}




\begin{tabular}{cccccccc}
$\mathrm{C}$ & 8.7749 & 12.4673 & 8.2332 & $\mathrm{H}$ & 13.8573 & 9.4303 & 9.6126 \\
$\mathrm{C}$ & 8.1962 & 13.4828 & 9.0288 & $\mathrm{H}$ & 12.2742 & 16.908 & 11.0037 \\
$\mathrm{C}$ & 9.7512 & 12.8076 & 7.31 & $\mathrm{H}$ & 10.9472 & 9.5322 & 8.8402 \\
$\mathrm{C}$ & 7.2013 & 13.1577 & 9.9801 & $\mathrm{H}$ & 12.0658 & 10.1473 & 7.6312 \\
$\mathrm{C}$ & 6.7133 & 11.7707 & 10.1002 & $\mathrm{H}$ & 13.9785 & 17.5052 & 9.36 \\
$\mathrm{C}$ & 8.3642 & 11.0713 & 8.381 & $\mathrm{H}$ & 16.8602 & 14.9404 & 6.4253 \\
$\mathrm{C}$ & 8.6178 & 14.8357 & 8.8961 & $\mathrm{H}$ & 14.3272 & 8.4894 & 7.5194 \\
$\mathrm{C}$ & 10.1462 & 14.1319 & 7.1282 & $\mathrm{H}$ & 13.1523 & 8.2823 & 6.2085 \\
$\mathrm{C}$ & 7.1222 & 15.4514 & 10.7157 & $\mathrm{H}$ & 10.1873 & 12.018 & 6.7063 \\
$\mathrm{C}$ & 9.5867 & 15.1599 & 7.8862 & $\mathrm{H}$ & 10.8902 & 14.3769 & 6.3746 \\
$\mathrm{C}$ & 6.6694 & 14.127 & 10.8079 & $\mathrm{H}$ & 6.7256 & 16.2069 & 11.3864 \\
\hline
\end{tabular}

\begin{tabular}{|c|c|c|c|c|c|c|c|}
\hline \multicolumn{4}{|c|}{ Cartesian coordinate, $\mathbf{6}, \mathrm{S} 0$, Gas State } & \multicolumn{4}{|c|}{ Cartesian coordinate, $\mathrm{Ag}^{\mathrm{I}}-\mathrm{NHC}_{2}-\mathrm{NH}, \mathrm{S} 0$, Gas State } \\
\hline & $\mathrm{x}$ & y & $\mathrm{z}$ & & $\mathrm{x}$ & $\mathrm{y}$ & $\mathrm{z}$ \\
\hline $\mathrm{H}$ & 5.9076 & 13.8349 & 11.5242 & $\mathrm{Ag}$ & 10.6975 & 7.1855 & 8.9338 \\
\hline $\mathrm{H}$ & 5.713 & 9.507 & 9.3605 & $\mathrm{C}$ & 8.0064 & 1.2177 & 8.7467 \\
\hline $\mathrm{H}$ & 7.1603 & 8.9211 & 8.4856 & $\mathrm{C}$ & 7.8835 & 0.2454 & 9.7614 \\
\hline $\mathrm{H}$ & 6.8383 & 9.2228 & 11.5148 & $\mathrm{C}$ & 7.8523 & 0.8019 & 7.398 \\
\hline $\mathrm{H}$ & 8.389 & 8.8969 & 10.7195 & $\mathrm{C}$ & 8.0119 & 0.6161 & 11.1257 \\
\hline $\mathrm{H}$ & 8.4286 & 16.8228 & 9.7444 & $\mathrm{C}$ & 8.226 & 2.0195 & 11.4926 \\
\hline $\mathrm{H}$ & 5.499 & 7.1299 & 12.0905 & $\mathrm{C}$ & 8.2408 & 2.5979 & 9.056 \\
\hline $\mathrm{H}$ & 4.9339 & 7.1578 & 10.4123 & $\mathrm{C}$ & 7.6154 & -1.1258 & 9.442 \\
\hline $\mathrm{H}$ & 15.7195 & 0.6562 & 7.7339 & $\mathrm{C}$ & 7.5837 & -0.4893 & 7.0534 \\
\hline $\mathrm{H}$ & 10.2434 & 0.3504 & 11.5783 & $\mathrm{C}$ & 7.6412 & -1.6577 & 11.829 \\
\hline $\mathrm{H}$ & 11.9778 & 4.4927 & 10.2206 & $\mathrm{C}$ & 7.447 & -1.5495 & 8.0269 \\
\hline $\mathrm{H}$ & 13.6074 & 4.5721 & 9.5075 & $\mathrm{C}$ & 14.8781 & 11.3989 & 8.7578 \\
\hline $\mathrm{H}$ & 10.558 & -2.1193 & 11.8798 & $\mathrm{C}$ & 14.5907 & 12.629 & 10.8968 \\
\hline $\mathrm{H}$ & 10.925 & 4.4553 & 8.0241 & $\mathrm{C}$ & 7.8935 & -0.3268 & 12.1403 \\
\hline $\mathrm{H}$ & 12.4382 & 4.0363 & 7.2359 & $\mathrm{C}$ & 8.358 & 4.3261 & 10.8016 \\
\hline $\mathrm{H}$ & 12.4562 & -3.2495 & 10.8433 & $\mathrm{C}$ & 15.2513 & 12.4524 & 9.656 \\
\hline $\mathrm{H}$ & 16.1179 & -1.7762 & 8.0246 & $\mathrm{C}$ & 9.7158 & 4.838 & 11.2973 \\
\hline $\mathrm{H}$ & 14.3996 & 6.0899 & 7.9318 & $\mathrm{C}$ & 13.5344 & 11.7643 & 11.276 \\
\hline $\mathrm{H}$ & 13.7629 & 5.9593 & 6.2844 & $\mathrm{C}$ & 13.1483 & 10.6484 & 10.405 \\
\hline $\mathrm{H}$ & 9.9434 & -4.1845 & 6.3939 & $\mathrm{C}$ & 7.5042 & -2.0339 & 10.4933 \\
\hline $\mathrm{H}$ & 11.1973 & -3.5306 & 5.4784 & $\mathrm{C}$ & 16.2904 & 13.351 & 9.3214 \\
\hline $\mathrm{H}$ & 15.5735 & -3.7506 & 9.1789 & $\mathrm{C}$ & 8.9514 & 6.6616 & 12.8968 \\
\hline $\mathrm{H}$ & 14.3414 & -3.8575 & 10.3253 & $\mathrm{C}$ & 9.6816 & 7.2487 & 10.7646 \\
\hline $\mathrm{H}$ & 15.7861 & 17.7163 & 8.3808 & $\mathrm{C}$ & 12.8592 & 11.9493 & 12.4797 \\
\hline $\mathrm{H}$ & 16.7016 & 17.1834 & 7.0728 & $\mathrm{C}$ & 13.5856 & 9.3489 & 8.4017 \\
\hline $\mathrm{H}$ & 10.552 & 16.6392 & 6.8861 & $\mathrm{C}$ & 14.9703 & 13.6772 & 11.7856 \\
\hline $\mathrm{H}$ & 9.3243 & 17.1892 & 7.9027 & $\mathrm{C}$ & 13.2206 & 12.9884 & 13.3329 \\
\hline $\mathrm{N}$ & 8.046 & 2.0042 & 8.5643 & $\mathrm{C}$ & 12.5059 & 9.5531 & 7.3359 \\
\hline $\mathrm{N}$ & 7.3132 & 5.2064 & 10.4032 & $\mathrm{C}$ & 14.269 & 13.8361 & 12.9811 \\
\hline $\mathrm{N}$ & 13.3224 & 11.415 & 9.4147 & $\mathrm{C}$ & 16.0889 & 14.5844 & 11.4515 \\
\hline $\mathrm{N}$ & 12.3224 & 8.1565 & 8.1275 & $\mathrm{C}$ & 16.6968 & 14.3534 & 10.1597 \\
\hline $\mathrm{N}$ & 7.3074 & 10.8216 & 9.2685 & $\mathrm{C}$ & 11.7586 & 7.2143 & 7.129 \\
\hline
\end{tabular}




\begin{tabular}{cccccccc}
$\mathrm{N}$ & 7.0289 & 7.3455 & 10.6446 & $\mathrm{C}$ & 12.8619 & 8.082 & 5.2678 \\
$\mathrm{~N}$ & 12.7194 & 2.7135 & 9.483 & $\mathrm{C}$ & 7.9038 & 11.9865 & 6.7932 \\
$\mathrm{~N}$ & 12.2911 & 6.0133 & 7.8071 & $\mathrm{C}$ & 6.867 & 12.8498 & 7.2094 \\
$\mathrm{~N}$ & 10.6277 & -3.4488 & 6.3077 & $\mathrm{C}$ & 8.544 & 12.2497 & 5.5535 \\
$\mathrm{~N}$ & 14.6724 & -3.4133 & 9.4832 & $\mathrm{C}$ & 6.2199 & 12.6392 & 8.4554 \\
$\mathrm{~N}$ & 15.8649 & 17.0557 & 7.6228 & $\mathrm{C}$ & 6.6106 & 11.5137 & 9.3129 \\
$\mathrm{~N}$ & 9.9887 & 16.4571 & 7.7034 & $\mathrm{C}$ & 8.2992 & 10.8625 & 7.5872 \\
$\mathrm{O}$ & 6.5583 & 1.2215 & 10.087 & $\mathrm{C}$ & 6.4511 & 13.9511 & 6.3926 \\
$\mathrm{O}$ & 9.6121 & 2.8133 & 7.1144 & $\mathrm{C}$ & 8.1797 & 13.2878 & 4.7494 \\
$\mathrm{O}$ & 14.874 & 10.737 & 7.8963 & $\mathrm{C}$ & 4.7927 & 14.5477 & 8.0897 \\
$\mathrm{O}$ & 11.7852 & 12.0639 & 10.9601 & $\mathrm{C}$ & 7.1119 & 14.2046 & 5.0839 \\
$\mathrm{O}$ & 5.8336 & 11.436 & 10.8786 & $\mathrm{C}$ & 5.2019 & 13.4827 & 8.8849 \\
\hline
\end{tabular}

\begin{tabular}{|c|c|c|c|c|c|c|c|}
\hline \multicolumn{8}{|c|}{ Cartesian coordinate, $\mathrm{Ag}^{\mathrm{I}}-\mathrm{NHC}_{2}-\mathrm{NH}, \mathrm{S} 0$, Gas State } \\
\hline & $\mathrm{x}$ & $\mathrm{y}$ & $\mathrm{z}$ & & $\mathrm{x}$ & $\mathrm{y}$ & $\mathrm{z}$ \\
\hline $\mathrm{C}$ & 7.8646 & 9.4557 & 9.55 & $\mathrm{H}$ & 4.744 & 13.2753 & 9.8477 \\
\hline $\mathrm{C}$ & 8.9819 & 9.6076 & 10.5823 & $\mathrm{H}$ & 6.9414 & 9.1618 & 10.0564 \\
\hline $\mathrm{C}$ & 5.4185 & 14.7638 & 6.8623 & $\mathrm{H}$ & 8.1555 & 8.6889 & 8.8295 \\
\hline $\mathrm{C}$ & 8.696 & 8.1595 & 12.6711 & $\mathrm{H}$ & 8.7117 & 10.3785 & 11.3094 \\
\hline $\mathrm{C}$ & 14.1622 & 2.2195 & 6.8198 & $\mathrm{H}$ & 9.9256 & 9.8935 & 10.1101 \\
\hline $\mathrm{C}$ & 13.7161 & 0.3121 & 8.3448 & $\mathrm{H}$ & 5.0821 & 15.5998 & 6.2505 \\
\hline $\mathrm{C}$ & 14.3982 & 0.8731 & 7.2388 & $\mathrm{H}$ & 9.2755 & 8.7893 & 13.3563 \\
\hline $\mathrm{C}$ & 12.7833 & 1.0905 & 9.0772 & $\mathrm{H}$ & 7.6401 & 8.44 & 12.7654 \\
\hline $\mathrm{C}$ & 12.5687 & 2.4974 & 8.7299 & $\mathrm{H}$ & 15.8595 & 0.5282 & 5.7127 \\
\hline $\mathrm{C}$ & 15.342 & 0.0685 & 6.5516 & $\mathrm{H}$ & 11.3512 & 1.1786 & 10.6537 \\
\hline $\mathrm{C}$ & 12.075 & 0.5495 & 10.1445 & $\mathrm{H}$ & 13.2172 & 4.9373 & 8.2809 \\
\hline $\mathrm{C}$ & 13.1298 & 4.3864 & 7.3388 & $\mathrm{H}$ & 13.9693 & 4.6305 & 6.6845 \\
\hline $\mathrm{C}$ & 13.9538 & -1.0392 & 8.7472 & $\mathrm{H}$ & 11.7472 & -1.1959 & 11.3617 \\
\hline $\mathrm{C}$ & 12.3057 & -0.7649 & 10.5353 & $\mathrm{H}$ & 10.9664 & 4.6604 & 7.3556 \\
\hline $\mathrm{C}$ & 11.8084 & 4.7964 & 6.6708 & $\mathrm{H}$ & 11.62 & 4.1896 & 5.7789 \\
\hline $\mathrm{C}$ & 13.2436 & -1.5292 & 9.8456 & $\mathrm{H}$ & 13.4261 & -2.549 & 10.1815 \\
\hline $\mathrm{C}$ & 14.9301 & -1.8813 & 8.008 & $\mathrm{H}$ & 16.335 & -1.8192 & 6.3742 \\
\hline $\mathrm{C}$ & 15.6095 & -1.2178 & 6.9161 & $\mathrm{H}$ & 13.5391 & 5.9983 & 4.9611 \\
\hline $\mathrm{C}$ & 12.6149 & 6.5795 & 5.0576 & $\mathrm{H}$ & 11.9959 & 6.377 & 4.1767 \\
\hline $\mathrm{H}$ & 7.9582 & 1.568 & 6.6335 & $\mathrm{H}$ & 7.1318 & -3.3984 & 8.4042 \\
\hline $\mathrm{H}$ & 7.4671 & -0.7835 & 6.0134 & $\mathrm{H}$ & 14.6478 & -3.4705 & 9.0326 \\
\hline $\mathrm{H}$ & 7.5425 & -2.4001 & 12.6175 & $\mathrm{H}$ & 17.2274 & 16.0249 & 11.9093 \\
\hline $\mathrm{H}$ & 7.9951 & 0.0179 & 13.166 & $\mathrm{H}$ & 6.0489 & 15.7212 & 4.6087 \\
\hline $\mathrm{H}$ & 7.6079 & 4.4859 & 11.5838 & $\mathrm{~N}$ & 8.3205 & 2.9216 & 10.4474 \\
\hline $\mathrm{H}$ & 8.0816 & 4.8778 & 9.901 & $\mathrm{~N}$ & 9.5977 & 6.2478 & 11.6469 \\
\hline $\mathrm{H}$ & 10.0326 & 4.2787 & 12.1826 & $\mathrm{~N}$ & 13.8532 & 10.521 & 9.2145 \\
\hline $\mathrm{H}$ & 10.4783 & 4.7165 & 10.5243 & $\mathrm{~N}$ & 12.3057 & 8.3094 & 6.6025 \\
\hline $\mathrm{H}$ & 7.2965 & -3.0788 & 10.266 & $\mathrm{~N}$ & 7.5865 & 10.669 & 8.805 \\
\hline $\mathrm{H}$ & 16.7751 & 13.2073 & 8.3584 & $\mathrm{~N}$ & 9.1665 & 8.3554 & 11.3008 \\
\hline $\mathrm{H}$ & 9.6213 & 6.4713 & 13.7424 & $\mathrm{~N}$ & 13.2516 & 2.9727 & 7.6199 \\
\hline $\mathrm{H}$ & 8.0304 & 6.0935 & 13.067 & $\mathrm{~N}$ & 11.8879 & 6.1951 & 6.2723 \\
\hline
\end{tabular}




\begin{tabular}{cccccccc}
$\mathrm{H}$ & 12.0553 & 11.2604 & 12.7237 & $\mathrm{~N}$ & 7.1884 & -2.7582 & 7.6086 \\
$\mathrm{H}$ & 13.2646 & 8.5499 & 9.0739 & $\mathrm{~N}$ & 15.2156 & -3.1309 & 8.253 \\
$\mathrm{H}$ & 14.5235 & 9.0723 & 7.9137 & $\mathrm{~N}$ & 16.4472 & 15.4986 & 12.3131 \\
$\mathrm{H}$ & 12.685 & 13.1402 & 14.2672 & $\mathrm{~N}$ & 6.816 & 15.1542 & 4.2396 \\
$\mathrm{H}$ & 11.554 & 9.8666 & 7.7739 & $\mathrm{O}$ & 8.2925 & 2.4088 & 12.664 \\
$\mathrm{H}$ & 12.8275 & 10.3205 & 6.6265 & $\mathrm{O}$ & 8.3354 & 3.5118 & 8.2317 \\
$\mathrm{H}$ & 14.5788 & 14.6507 & 13.6306 & $\mathrm{O}$ & 15.3759 & 11.1839 & 7.6469 \\
$\mathrm{H}$ & 17.5106 & 15.016 & 9.863 & $\mathrm{O}$ & 12.2642 & 9.8396 & 10.702 \\
$\mathrm{H}$ & 13.9225 & 8.3575 & 5.2359 & $\mathrm{O}$ & 6.1023 & 11.2937 & 10.416 \\
$\mathrm{H}$ & 12.3294 & 8.6986 & 4.5348 & $\mathrm{O}$ & 9.1851 & 10.0502 & 7.2939 \\
$\mathrm{H}$ & 9.347 & 11.5774 & 5.261 & $\mathrm{O}$ & 14.6935 & 2.7757 & 5.8496 \\
$\mathrm{H}$ & 8.6858 & 13.4736 & 3.8061 & $\mathrm{O}$ & 11.8445 & 3.2554 & 9.3788 \\
$\mathrm{H}$ & 3.9953 & 15.2086 & 8.422 & & & & \\
\hline
\end{tabular}

Cartesian coordinate, 4-dimethylamine derivative of 3, S0, Gas State

\begin{tabular}{cccccccc} 
& $\mathrm{x}$ & $\mathrm{y}$ & $\mathrm{z}$ & & $\mathrm{x}$ & $\mathrm{y}$ & $\mathrm{z}$ \\
\hline $\mathrm{Ag}$ & 10.2407 & 6.655 & 8.9967 & $\mathrm{C}$ & 7.522405 & 0.189755 & 10.23407 \\
$\mathrm{C}$ & 8.839085 & 3.945806 & 8.460532 & $\mathrm{C}$ & 7.723127 & 1.639977 & 10.3294 \\
$\mathrm{C}$ & 6.4914 & 4.8431 & 8.5256 & $\mathrm{C}$ & 10.04989 & 1.350724 & 9.626625 \\
$\mathrm{C}$ & 8.2788 & 6.2336 & 8.9948 & $\mathrm{C}$ & 8.381943 & -2.02934 & 9.742597 \\
$\mathrm{C}$ & 12.1797 & 7.134 & 8.8315 & $\mathrm{C}$ & 10.65866 & -2.27187 & 8.971601 \\
$\mathrm{C}$ & 14.2273 & 8.1347 & 8.4047 & $\mathrm{C}$ & 6.142813 & -1.72511 & 10.59785 \\
$\mathrm{C}$ & 5.978 & 6.2465 & 8.7744 & $\mathrm{C}$ & 9.452476 & -2.85761 & 9.271629 \\
$\mathrm{C}$ & 13.12123 & 4.790313 & 8.636721 & $\mathrm{C}$ & 6.321939 & -0.33939 & 10.60312 \\
$\mathrm{C}$ & 14.3886 & 6.629 & 8.3451 & $\mathrm{C}$ & 9.147552 & 3.558401 & 9.918739 \\
$\mathrm{C}$ & 6.1148 & 12.2841 & 11.1051 & $\mathrm{C}$ & 7.154284 & -2.54692 & 10.19864 \\
$\mathrm{C}$ & 4.782 & 12.4394 & 10.6795 & $\mathrm{C}$ & 15.0789 & 3.522458 & 5.606679 \\
$\mathrm{C}$ & 6.8649 & 13.3906 & 11.3668 & $\mathrm{C}$ & 14.55726 & 1.314474 & 4.586782 \\
$\mathrm{C}$ & 4.0036 & 11.3044 & 10.3911 & $\mathrm{C}$ & 15.4039 & 2.433979 & 4.673728 \\
$\mathrm{C}$ & 4.5736 & 9.9617 & 10.5485 & $\mathrm{C}$ & 13.35718 & 1.277921 & 5.321216 \\
$\mathrm{C}$ & 6.7066 & 10.9599 & 11.2158 & $\mathrm{C}$ & 12.97877 & 2.405075 & 6.181059 \\
$\mathrm{C}$ & 4.2384 & 13.7204 & 10.5031 & $\mathrm{C}$ & 16.52969 & 2.516964 & 3.909421 \\
$\mathrm{C}$ & 6.3313 & 14.6722 & 11.2451 & $\mathrm{C}$ & 12.53574 & 0.19525 & 5.213505 \\
$\mathrm{C}$ & 2.2022 & 12.706 & 9.6891 & $\mathrm{C}$ & 14.89362 & 0.266208 & 3.724287 \\
$\mathrm{C}$ & 5.0338 & 14.8651 & 10.8361 & $\mathrm{C}$ & 12.8625 & -0.86496 & 4.354209 \\
$\mathrm{C}$ & 2.7299 & 11.4337 & 9.9239 & $\mathrm{C}$ & 14.00363 & -0.8377 & 3.598422 \\
$\mathrm{C}$ & 6.4769 & 8.5663 & 11.0395 & $\mathrm{C}$ & 16.06222 & 0.38256 & 2.94163 \\
$\mathrm{C}$ & 2.9462 & 13.8174 & 9.9519 & $\mathrm{C}$ & 16.86015 & 1.481214 & 3.020359 \\
$\mathrm{C}$ & 13.2812 & 12.3737 & 7.87 & $\mathrm{C}$ & 13.18874 & -2.36767 & 1.916123 \\
$\mathrm{C}$ & 11.9927 & 14.43 & 7.3233 & $\mathrm{C}$ & 15.39562 & -2.70894 & 2.843803 \\
$\mathrm{C}$ & 13.1975 & 13.8354 & 7.7387 & $\mathrm{C}$ & 8.12167 & -4.7356 & 8.457474 \\
$\mathrm{C}$ & 10.8466 & 13.638 & 7.123 & $\mathrm{C}$ & 10.45133 & -5.02215 & 8.996128 \\
$\mathrm{C}$ & 10.8978 & 12.1887 & 7.3464 & $\mathrm{C}$ & 13.53205 & 4.569795 & 7.169 \\
$\mathrm{C}$ & 14.2934 & 14.599 & 8.0114 & $\mathrm{H}$ & 6.2512 & 4.1724 & 9.3385 \\
$\mathrm{C}$ & 9.6797 & 14.229 & 6.7388 & $\mathrm{H}$ & 6.1334 & 4.404 & 7.6111
\end{tabular}




\begin{tabular}{cccccccc}
$\mathrm{C}$ & 12.2662 & 10.2053 & 7.739 & $\mathrm{H}$ & 14.9041 & 8.6094 & 9.0947 \\
$\mathrm{C}$ & 11.9326 & 15.8182 & 7.1646 & $\mathrm{H}$ & 14.3434 & 8.6062 & 7.4381 \\
$\mathrm{C}$ & 9.6061 & 15.6205 & 6.5729 & $\mathrm{H}$ & 5.187 & 6.2899 & 9.5034 \\
$\mathrm{C}$ & 10.6948 & 16.419 & 6.7993 & $\mathrm{H}$ & 5.6374 & 6.7287 & 7.8676 \\
$\mathrm{C}$ & 13.0813 & 16.5832 & 7.4595 & $\mathrm{H}$ & 15.0012 & 6.2451 & 9.1448 \\
$\mathrm{C}$ & 14.2334 & 15.9961 & 7.8813 & $\mathrm{H}$ & 14.803 & 6.2811 & 7.4147 \\
$\mathrm{C}$ & 9.4123 & 18.4173 & 7.2431 & $\mathrm{H}$ & 7.8756 & 13.2646 & 11.691 \\
$\mathrm{C}$ & 11.2957 & 18.5421 & 5.7316 & $\mathrm{H}$ & 6.9502 & 15.5063 & 11.4815 \\
$\mathrm{C}$ & 3.2241 & 16.4365 & 11.3013 & $\mathrm{H}$ & 1.2203 & 12.8043 & 9.2733 \\
$\mathrm{C}$ & 5.4289 & 17.2304 & 10.7462 & $\mathrm{H}$ & 2.1659 & 10.5489 & 9.712 \\
$\mathrm{C}$ & 12.5908 & 9.6663 & 9.1446 & $\mathrm{H}$ & 5.7074 & 7.8387 & 11.1922 \\
$\mathrm{C}$ & 7.2166 & 8.2627 & 9.7233 & $\mathrm{H}$ & 7.1694 & 8.5295 & 11.8544 \\
$\mathrm{C}$ & 9.823786 & -0.07945 & 9.487117 & $\mathrm{H}$ & 2.5506 & 14.7818 & 9.7228 \\
$\mathrm{C}$ & 8.57352 & -0.6413 & 9.807687 & $\mathrm{H}$ & 15.1914 & 14.1126 & 8.3265 \\
$\mathrm{C}$ & 10.83985 & -0.89522 & 9.090122 & $\mathrm{H}$ & 8.8213 & 13.6137 & 6.5677 \\
\hline
\end{tabular}

Cartesian coordinate, 2c, S0, Gas State

\begin{tabular}{cccccccc} 
& $\mathrm{x}$ & $\mathrm{y}$ & $\mathrm{z}$ & & $\mathrm{x}$ & $\mathrm{y}$ & $\mathrm{Z}$ \\
\hline $\mathrm{C}$ & 0.5611 & 2.1382 & -0.9219 & $\mathrm{H}$ & -1.7956 & 1.6374 & 3.7536 \\
$\mathrm{C}$ & -0.6543 & 2.4186 & -0.2385 & $\mathrm{H}$ & -2.4038 & -0.5181 & 2.5963 \\
$\mathrm{C}$ & 1.7323 & 2.8054 & -0.5474 & $\mathrm{H}$ & 0.7779 & -4.9631 & 1.3425 \\
$\mathrm{C}$ & -1.8502 & 1.7264 & -0.5864 & $\mathrm{H}$ & -0.9622 & -4.5804 & 1.1125 \\
$\mathrm{C}$ & -1.8653 & 0.7889 & -1.7304 & $\mathrm{H}$ & -0.3216 & -5.9329 & -0.7978 \\
$\mathrm{C}$ & 0.5828 & 1.2402 & -2.0791 & $\mathrm{H}$ & 1.2378 & -5.07 & -0.9819 \\
$\mathrm{C}$ & -0.6914 & 3.3988 & 0.8104 & $\mathrm{H}$ & 0.4989 & 2.5009 & 3.6377 \\
$\mathrm{C}$ & 1.7181 & 3.7664 & 0.4685 & $\mathrm{H}$ & -2.4116 & -4.7163 & -2.0774 \\
$\mathrm{C}$ & -3.0782 & 2.9342 & 1.1252 & $\mathrm{H}$ & -2.2391 & -3.4017 & -0.8536 \\
$\mathrm{C}$ & 0.5341 & 4.0813 & 1.1562 & $\mathrm{H}$ & 1.3431 & -2.9841 & -2.2259 \\
$\mathrm{C}$ & -3.0423 & 1.9737 & 0.0945 & $\mathrm{H}$ & -0.2895 & 5.4986 & 2.408 \\
$\mathrm{C}$ & -0.7366 & 0.0936 & -3.8092 & $\mathrm{H}$ & 1.393 & 5.5767 & 2.2475 \\
$\mathrm{C}$ & -0.0204 & -1.2531 & -3.94 & $\mathrm{H}$ & 2.4858 & 2.8046 & 3.7781 \\
$\mathrm{C}$ & -1.9256 & 3.6344 & 1.4704 & $\mathrm{H}$ & 4.0562 & 2.589 & 3.1339 \\
$\mathrm{C}$ & -1.9262 & -2.7234 & -2.9451 & $\mathrm{~N}$ & -0.6702 & 0.6944 & -2.4729 \\
$\mathrm{C}$ & 0.2577 & -3.0845 & -2.3097 & $\mathrm{~N}$ & -0.5136 & -2.2546 & -2.9996 \\
$\mathrm{C}$ & 1.9203 & -1.1574 & 1.1194 & $\mathrm{~N}$ & 0.3022 & -2.9769 & 0.8679 \\
$\mathrm{C}$ & 0.9376 & -0.4012 & 1.8196 & $\mathrm{~N}$ & -0.4159 & -4.0565 & -1.7047 \\
$\mathrm{C}$ & 3.222 & -0.6511 & 1.0044 & $\mathrm{~N}$ & 0.5659 & 4.98 & 2.2106 \\
$\mathrm{C}$ & -0.4075 & -0.8644 & 1.8897 & $\mathrm{~N}$ & 3.0554 & 2.4385 & 3.0193 \\
$\mathrm{C}$ & -0.7762 & -2.1731 & 1.3161 & $\mathrm{O}$ & -2.8762 & 0.1548 & -2.0712 \\
$\mathrm{C}$ & 1.6124 & -2.4925 & 0.6106 & $\mathrm{O}$ & 1.5924 & 0.9757 & -2.747 \\
$\mathrm{C}$ & 1.2874 & 0.8328 & 2.4645 & $\mathrm{O}$ & -1.9401 & -2.598 & 1.2685 \\
$\mathrm{C}$ & 3.5848 & 0.5513 & 1.6174 & $\mathrm{O}$ & 2.4157 & -3.2196 & -0.0016 \\
$\mathrm{C}$ & -1.0338 & 1.068 & 3.2166 & & & & \\
$\mathrm{C}$ & 2.6599 & 1.2847 & 2.3867 & & & & \\
$\mathrm{C}$ & -1.3827 & -0.1296 & 2.5672 & & & &
\end{tabular}




\begin{tabular}{lccc}
$\mathrm{C}$ & 0.0508 & -4.4161 & 0.7195 \\
$\mathrm{C}$ & 0.1778 & -4.9531 & -0.7211 \\
$\mathrm{C}$ & 0.2754 & 1.541 & 3.1616 \\
$\mathrm{C}$ & -1.8754 & -3.7899 & -1.8225 \\
$\mathrm{H}$ & 2.6543 & 2.5777 & -1.0884 \\
$\mathrm{H}$ & 2.644 & 4.2795 & 0.7435 \\
$\mathrm{H}$ & -4.0152 & 3.1325 & 1.6526 \\
$\mathrm{H}$ & -3.9379 & 1.4227 & -0.203 \\
$\mathrm{H}$ & -0.2492 & 0.7837 & -4.517 \\
$\mathrm{H}$ & -1.8036 & 0.0099 & -4.0614 \\
$\mathrm{H}$ & -0.1543 & -1.6286 & -4.9737 \\
$\mathrm{H}$ & 1.053 & -1.1073 & -3.7487 \\
$\mathrm{H}$ & -1.9842 & 4.3573 & 2.2909 \\
$\mathrm{H}$ & -2.1947 & -3.1488 & -3.9297 \\
$\mathrm{H}$ & -2.5875 & -1.8751 & -2.7084 \\
$\mathrm{H}$ & 3.962 & -1.2393 & 0.4549 \\
$\mathrm{C}$ & 0.5611 & 2.1382 & -0.9219 \\
\hline
\end{tabular}

Cartesian coordinate, 2c, S1, Gas State

\begin{tabular}{cccccccc} 
& $\mathrm{x}$ & $\mathrm{y}$ & $\mathrm{z}$ & & $\mathrm{x}$ & $\mathrm{y}$ & $\mathrm{z}$ \\
\hline $\mathrm{C}$ & 0.1166 & 5.7174 & 3.8117 & $\mathrm{H}$ & 1.9435 & -7.3269 & -6.9955 \\
$\mathrm{C}$ & -1.0257 & 5.3355 & 4.5489 & $\mathrm{H}$ & 2.3089 & -4.9418 & -6.3025 \\
$\mathrm{C}$ & 0.9345 & 6.7606 & 4.2573 & $\mathrm{H}$ & 0.8861 & -1.1991 & -3.7413 \\
$\mathrm{C}$ & -1.8267 & 4.2914 & 4.0566 & $\mathrm{H}$ & -0.251 & -1.3973 & -2.3755 \\
$\mathrm{C}$ & -1.4902 & 3.5986 & 2.7773 & $\mathrm{H}$ & 2.7556 & -1.951 & -2.3255 \\
$\mathrm{C}$ & 0.4862 & 5.0241 & 2.5398 & $\mathrm{H}$ & 1.6327 & -2.4392 & -1.05 \\
$\mathrm{C}$ & -1.3695 & 5.9891 & 5.7602 & $\mathrm{H}$ & 0.3282 & -8.7161 & -5.8054 \\
$\mathrm{C}$ & 0.6345 & 7.4208 & 5.4274 & $\mathrm{H}$ & 3.4366 & 0.6217 & -2.2821 \\
$\mathrm{C}$ & -3.2966 & 4.5312 & 5.9464 & $\mathrm{H}$ & 1.7728 & 0.9228 & -2.8486 \\
$\mathrm{C}$ & -0.5017 & 7.0569 & 6.2059 & $\mathrm{H}$ & 0.7472 & -0.5667 & 0.5555 \\
$\mathrm{C}$ & -2.9545 & 3.8902 & 4.753 & $\mathrm{H}$ & -2.3845 & -9.6297 & -3.6738 \\
$\mathrm{C}$ & -0.0373 & 3.3575 & 0.8187 & $\mathrm{H}$ & -1.4068 & -9.4848 & -5.0354 \\
$\mathrm{C}$ & 0.6922 & 2.0309 & 1.0143 & $\mathrm{H}$ & -0.1226 & 8.4651 & 7.6356 \\
$\mathrm{C}$ & -2.5179 & 5.5691 & 6.4446 & $\mathrm{H}$ & -1.5163 & 7.5068 & 7.9398 \\
$\mathrm{C}$ & 2.2106 & 1.858 & -0.9432 & $\mathrm{~N}$ & -0.3535 & 4.0207 & 2.0945 \\
$\mathrm{C}$ & 0.9395 & 0.0213 & -0.34 & $\mathrm{~N}$ & 0.9896 & 1.4318 & -0.2619 \\
$\mathrm{C}$ & -0.7395 & -5.2683 & -2.915 & $\mathrm{~N}$ & 0.4167 & -3.1487 & -3.2525 \\
$\mathrm{C}$ & -0.0566 & -5.8189 & -4.0208 & $\mathrm{~N}$ & 1.955 & -0.408 & -1.1932 \\
$\mathrm{C}$ & -1.6582 & -6.0428 & -2.23 & $\mathrm{~N}$ & -1.5515 & -9.2227 & -4.073 \\
$\mathrm{C}$ & 0.8874 & -5.038 & -4.7274 & $\mathrm{~N}$ & -0.7349 & 7.7177 & 7.3366 \\
$\mathrm{C}$ & 1.1413 & -3.6354 & -4.3356 & $\mathrm{O}$ & -2.1946 & 2.7076 & 2.3586 \\
$\mathrm{C}$ & -0.4748 & -3.8978 & -2.4746 & $\mathrm{O}$ & 1.4861 & 5.3635 & 1.943 \\
$\mathrm{C}$ & -0.2981 & -7.1614 & -4.4257 & $\mathrm{O}$ & 1.9426 & -2.9224 & -4.9199 \\
$\mathrm{C}$ & -1.9386 & -7.3478 & -2.6294 & $\mathrm{O}$ & -0.9903 & -3.3999 & -1.4855 \\
$\mathrm{C}$ & 1.3771 & -6.9044 & -6.1715 & & & & \\
$\mathrm{C}$ & -1.2866 & -7.9248 & -3.7164 & & & & \\
$\mathrm{C}$ & 1.591 & -5.5723 & -5.7874 & & & &
\end{tabular}




\begin{tabular}{|c|c|c|c|}
\hline $\mathrm{C}$ & 0.6616 & -1.7717 & -2.8404 \\
\hline $\mathrm{C}$ & 1.81 & -1.6976 & -1.836 \\
\hline $\mathrm{C}$ & 0.4532 & -7.6803 & -5.5033 \\
\hline $\mathrm{C}$ & 2.3986 & 0.7419 & -1.9624 \\
\hline $\mathrm{H}$ & 1.8016 & 7.0267 & 3.663 \\
\hline $\mathrm{H}$ & 1.2681 & 8.2298 & 5.7774 \\
\hline $\mathrm{H}$ & -4.1814 & 4.2194 & 6.4905 \\
\hline $\mathrm{H}$ & -3.5538 & 3.081 & 4.35 \\
\hline $\mathrm{H}$ & 0.5641 & 4.058 & 0.24 \\
\hline $\mathrm{H}$ & -0.9787 & 3.1826 & 0.2967 \\
\hline $\mathrm{H}$ & 1.6014 & 2.1795 & 1.6205 \\
\hline $\mathrm{H}$ & 0.0341 & 1.3547 & 1.5662 \\
\hline $\mathrm{H}$ & -2.8281 & 6.0379 & 7.3737 \\
\hline $\mathrm{H}$ & 3.0651 & 1.8843 & -0.2464 \\
\hline $\mathrm{H}$ & 2.1014 & 2.8406 & -1.4083 \\
\hline $\mathrm{H}$ & -2.1683 & -5.6025 & -1.3789 \\
\hline $\mathrm{H}$ & -2.6828 & -7.9282 & -2.0898 \\
\hline
\end{tabular}

Cartesian coordinate, 2a, S1, Gas State

\begin{tabular}{cccccccc} 
& $\mathrm{x}$ & $\mathrm{y}$ & $\mathrm{z}$ & & $\mathrm{x}$ & $\mathrm{y}$ & $\mathrm{z}$ \\
\hline $\mathrm{C}$ & -0.1184 & 5.7802 & 3.5306 & $\mathrm{H}$ & 1.5421 & -6.8108 & -7.4209 \\
$\mathrm{C}$ & -1.0769 & 5.2665 & 4.4144 & $\mathrm{H}$ & 2.064 & -4.6063 & -6.4689 \\
$\mathrm{C}$ & 0.3995 & 7.0654 & 3.6976 & $\mathrm{H}$ & 0.8034 & -1.0777 & -3.3531 \\
$\mathrm{C}$ & -1.5557 & 3.9533 & 4.275 & $\mathrm{H}$ & -0.3184 & -1.3807 & -2.0394 \\
$\mathrm{C}$ & -1.0689 & 3.1075 & 3.1616 & $\mathrm{H}$ & 2.6566 & -2.1804 & -2.0361 \\
$\mathrm{C}$ & 0.3798 & 4.9439 & 2.4153 & $\mathrm{H}$ & 1.5309 & -2.5032 & -0.7201 \\
$\mathrm{C}$ & -1.5464 & 6.0762 & 5.483 & $\mathrm{H}$ & -0.0676 & -8.3214 & -6.382 \\
$\mathrm{C}$ & -0.0612 & 7.8635 & 4.7396 & $\mathrm{H}$ & 3.9566 & 0.0316 & -1.6082 \\
$\mathrm{C}$ & -2.9347 & 4.1919 & 6.2343 & $\mathrm{H}$ & 2.6425 & 0.7207 & -2.6204 \\
$\mathrm{C}$ & -1.0682 & 7.4117 & 5.6029 & $\mathrm{H}$ & 0.7244 & -0.504 & 0.6824 \\
$\mathrm{C}$ & -2.4794 & 3.4169 & 5.1772 & $\mathrm{H}$ & -1.4745 & 8.0653 & 6.3463 \\
$\mathrm{C}$ & 0.2177 & 2.8985 & 1.1092 & $\mathrm{H}$ & -1.8975 & -8.7318 & -4.4475 \\
$\mathrm{C}$ & 1.4481 & 2.0641 & 1.4794 & $\mathrm{~N}$ & -0.2467 & 3.718 & 2.2207 \\
$\mathrm{C}$ & -2.4729 & 5.501 & 6.3842 & $\mathrm{~N}$ & 1.8647 & 1.2422 & 0.339 \\
$\mathrm{C}$ & 2.8615 & 1.6819 & -0.6559 & $\mathrm{~N}$ & 0.2755 & -3.0898 & -3.0957 \\
$\mathrm{C}$ & 1.4488 & 0.0255 & 0.0738 & $\mathrm{~N}$ & 1.9957 & -0.4827 & -1.0061 \\
$\mathrm{C}$ & -0.8971 & -5.2416 & -2.8969 & $\mathrm{O}$ & -1.3504 & 1.9127 & 3.1094 \\
$\mathrm{C}$ & -0.2646 & -5.6815 & -4.1474 & $\mathrm{O}$ & 1.3303 & 5.3122 & 1.7296 \\
$\mathrm{C}$ & -1.783 & -6.1004 & -2.2608 & $\mathrm{O}$ & 1.9481 & -2.7941 & -4.6588 \\
$\mathrm{C}$ & 0.7153 & -4.8027 & -4.8043 & $\mathrm{O}$ & -0.9595 & -3.5899 & -1.2109 \\
$\mathrm{C}$ & 1.0334 & -3.4823 & -4.2102 & & & & \\
$\mathrm{C}$ & -0.5571 & -3.917 & -2.3257 & & & & \\
$\mathrm{C}$ & -0.5929 & -7.0192 & -4.7281 & & & & \\
$\mathrm{C}$ & -2.0877 & -7.3521 & -2.8083 & & & & \\
$\mathrm{C}$ & 1.0358 & -6.4921 & -6.5104 & & & & \\
$\mathrm{C}$ & -1.5658 & -7.8023 & -4.0342 & & & &
\end{tabular}




\begin{tabular}{lccc}
$\mathrm{C}$ & 1.3338 & -5.2401 & -5.9698 \\
$\mathrm{C}$ & 0.5738 & -1.7769 & -2.5398 \\
$\mathrm{C}$ & 1.7429 & -1.8203 & -1.5505 \\
$\mathrm{C}$ & 0.1073 & -7.3553 & -5.9144 \\
$\mathrm{C}$ & 2.9536 & 0.4676 & -1.603 \\
$\mathrm{H}$ & 1.1656 & 7.4503 & 3.0273 \\
$\mathrm{H}$ & 0.3688 & 8.8544 & 4.8718 \\
$\mathrm{H}$ & -3.646 & 3.7814 & 6.948 \\
$\mathrm{H}$ & -2.8416 & 2.3968 & 5.0644 \\
$\mathrm{H}$ & 0.452 & 3.544 & 0.254 \\
$\mathrm{H}$ & -0.5937 & 2.2358 & 0.7839 \\
$\mathrm{H}$ & 2.2855 & 2.7062 & 1.7741 \\
$\mathrm{H}$ & 1.2362 & 1.4045 & 2.3285 \\
$\mathrm{H}$ & -2.8419 & 6.068 & 7.2361 \\
$\mathrm{H}$ & 3.8159 & 1.8854 & -0.1622 \\
$\mathrm{H}$ & 2.5019 & 2.5754 & -1.1739 \\
$\mathrm{H}$ & -2.2414 & -5.8094 & -1.3179 \\
$\mathrm{H}$ & -2.7676 & -7.9972 & -2.2526 \\
\hline
\end{tabular}

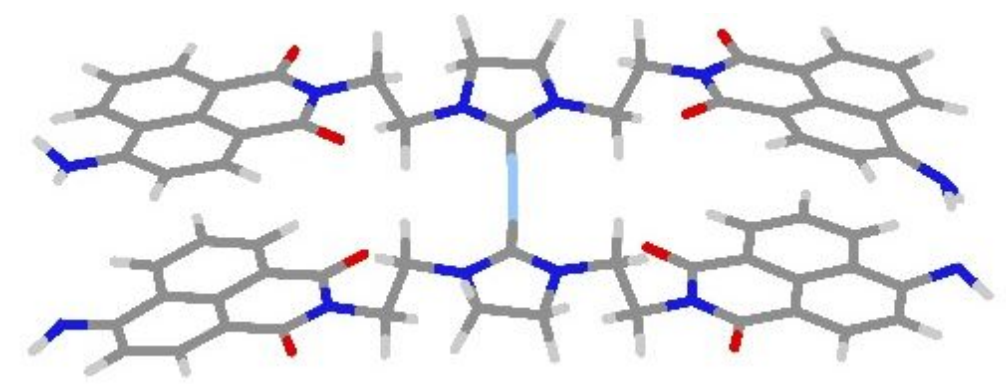

Figure S39. Molecular view of optimized geometries of 6 in First Excited-State $\left(\mathrm{S}_{1}\right)$.

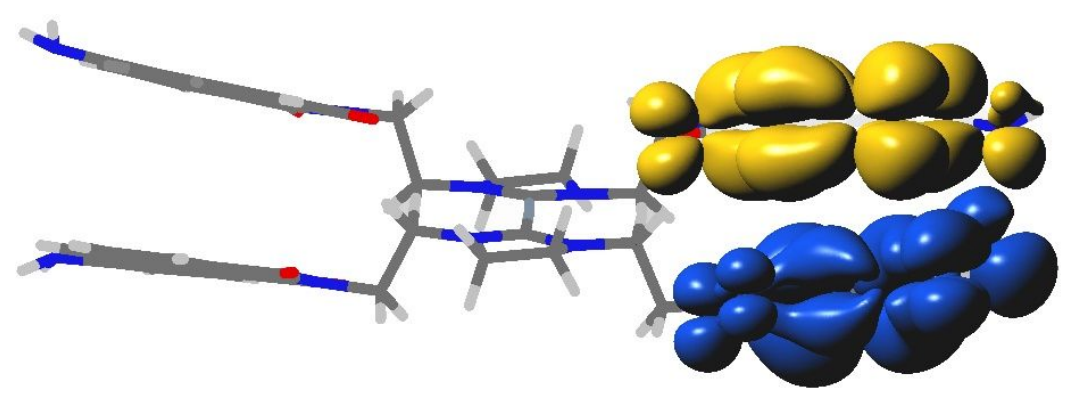

Figure S40. PBE0-DFT transition densities for $\mathrm{S}_{1} \rightarrow \mathrm{S}_{0}$ of $\mathbf{6}$. During the electronic transition, the electron density increases in the yellow areas and decreases in the blue areas. 


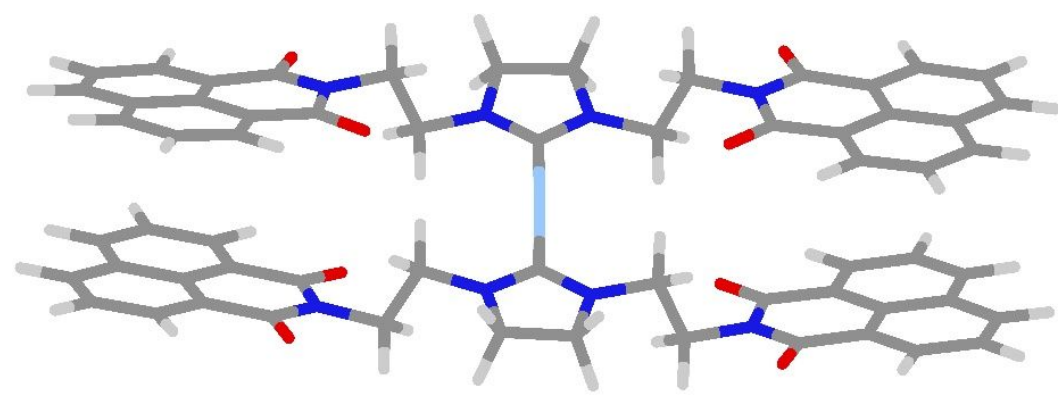

Figure S41. Molecular view of optimized geometries of $\mathbf{3}$ in First Excited-State $\left(\mathrm{S}_{1}\right)$.

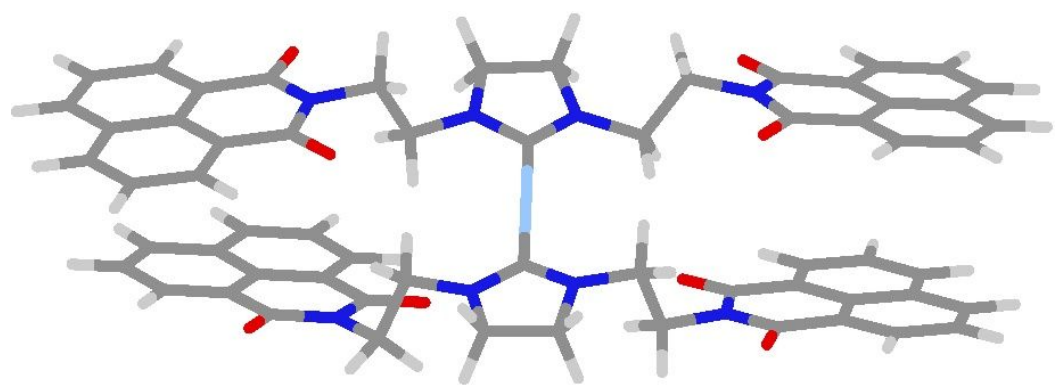

Figure S42. Molecular view of optimized geometries of $\mathbf{3}$ in ground-state $\left(\mathrm{S}_{0}\right)$ in DCM solvent.

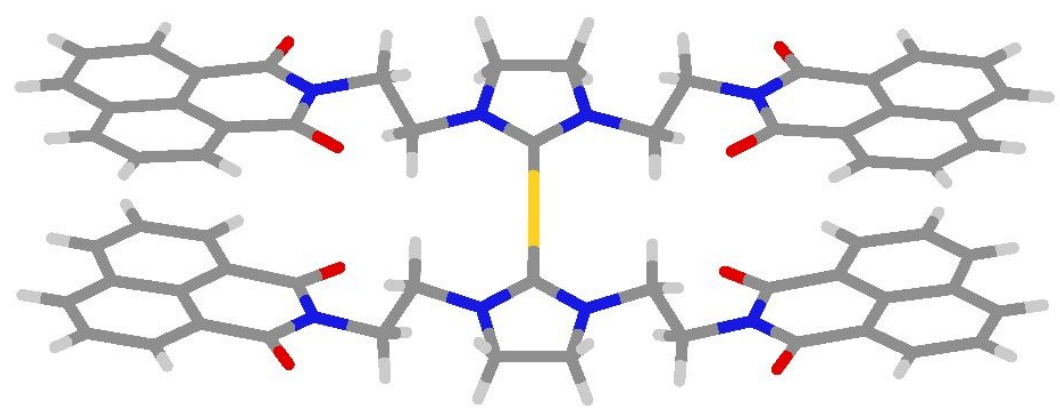

Figure S43. Molecular view of optimized geometries of 4 in First Excited-State $\left(S_{1}\right)$.

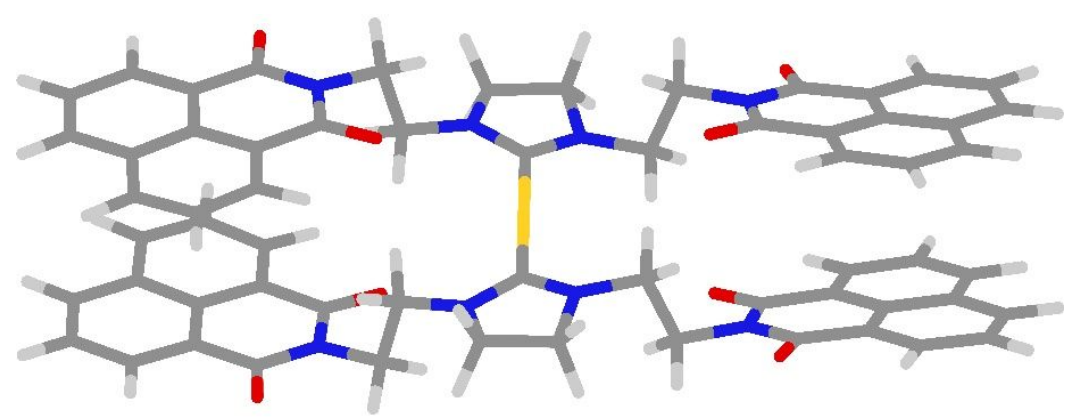

Figure S44. Molecular view of optimized geometries of $\mathbf{4}$ in ground-state $\left(\mathrm{S}_{0}\right)$ in DCM solvent. 


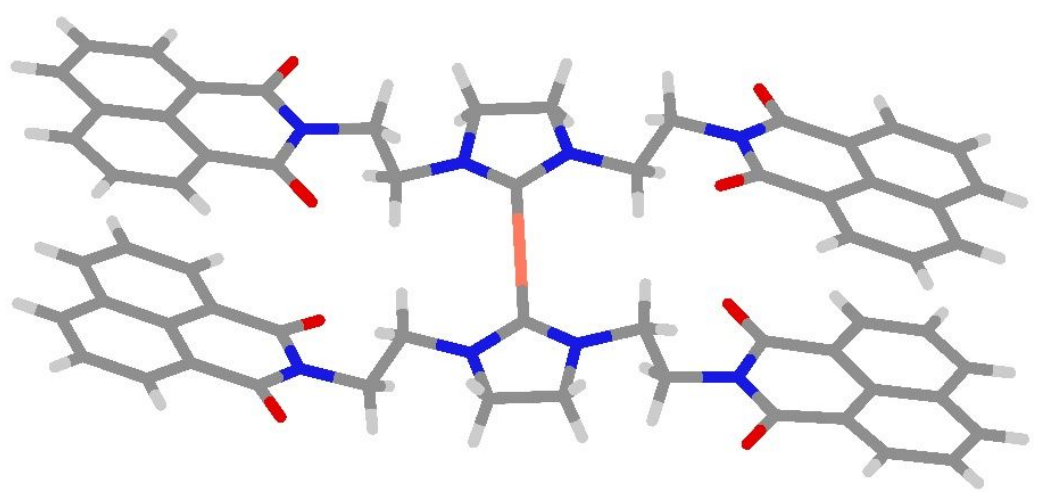

Figure S45. Molecular view of optimized geometries of 5 in First Excited-State $\left(\mathrm{S}_{1}\right)$.

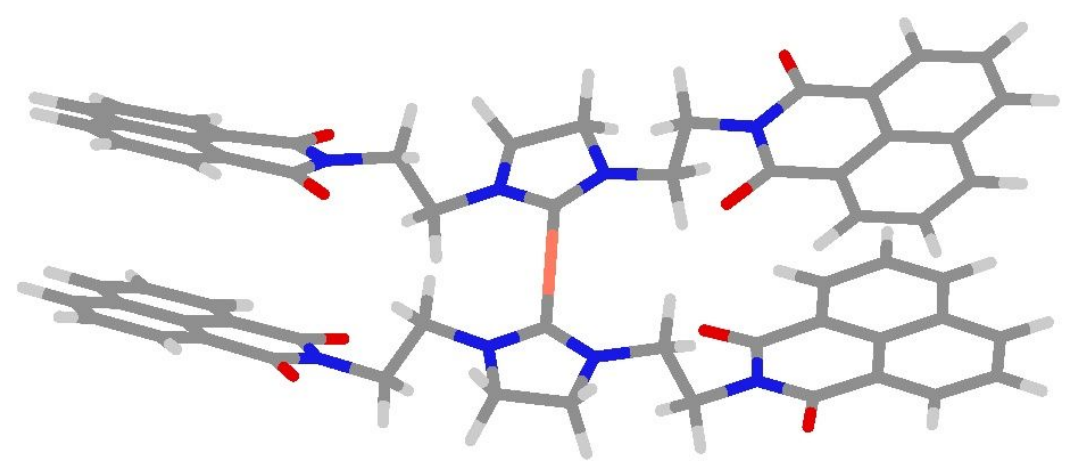

Figure S46. Molecular view of optimized geometries of 5 in ground-state $\left(\mathrm{S}_{0}\right)$ in DCM solvent.

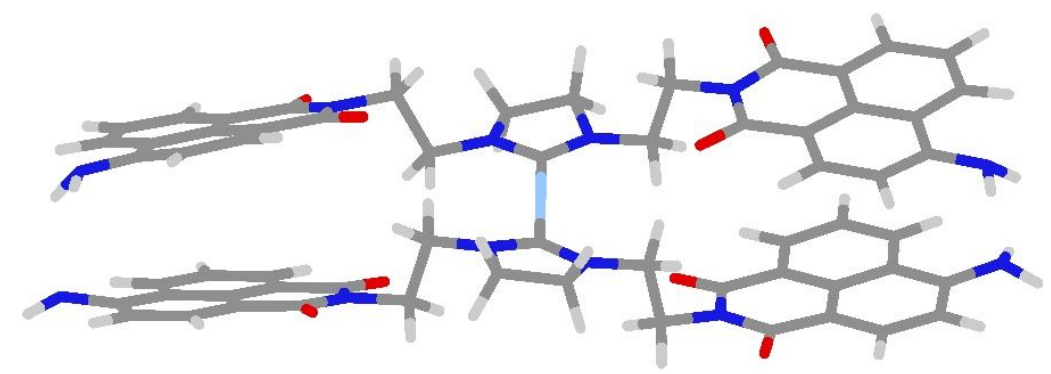

Figure S47. Molecular view of optimized geometries 6 in ground-state $\left(\mathrm{S}_{0}\right)$ in DCM solvent. 
(3)

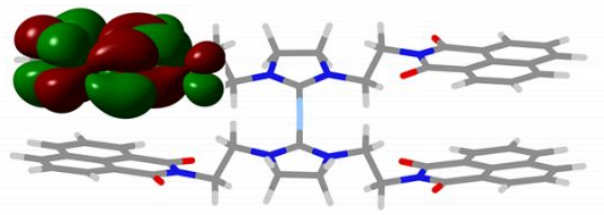

hole

(4)

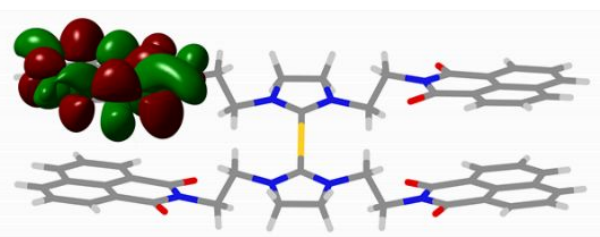

hole

(5)

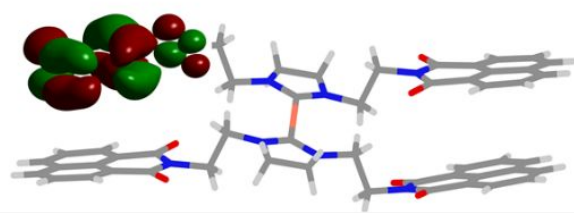

hole

(6)

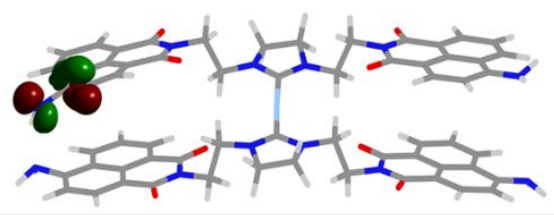

hole

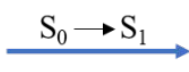

$\mathrm{S}_{0} \rightarrow \mathrm{S}_{1}$

$\mathrm{S}_{0 \rightarrow \mathrm{S}_{1}}$

$\stackrel{\mathrm{S}_{0} \rightarrow \mathrm{S}_{1}}{\longrightarrow}$

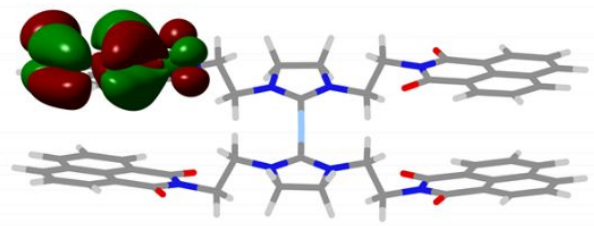

particle

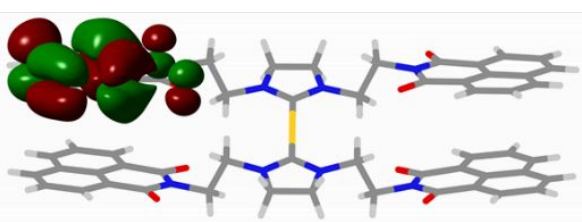

particle
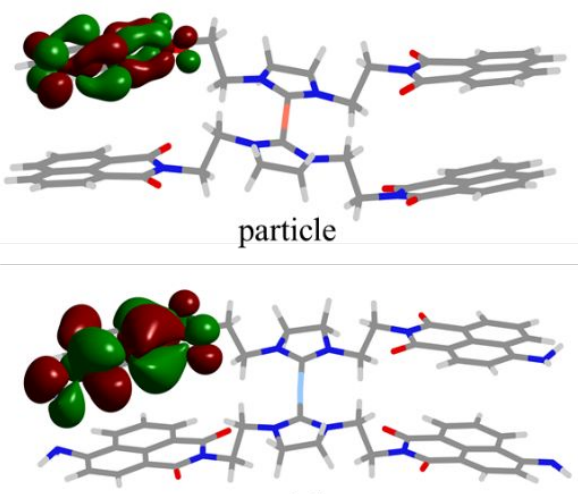

particle

Figure S48: The NTO calculations, $\mathrm{S} 0 \rightarrow \mathrm{S} 1$ transitions of 3-6.

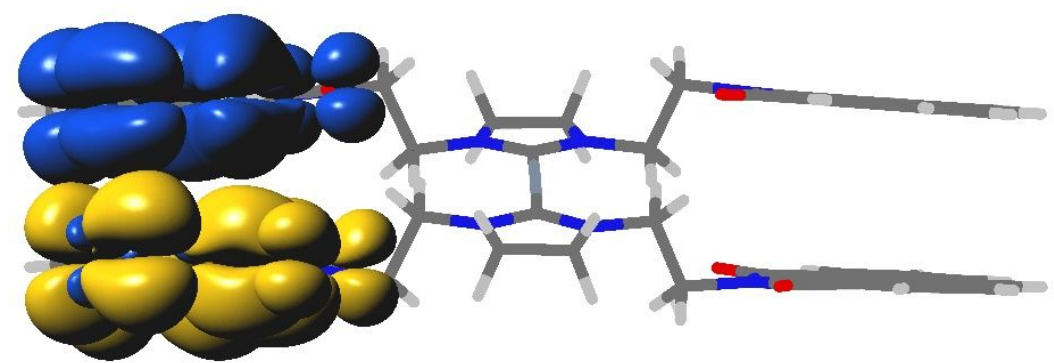

Figure S49. PBE0-DFT transition densities for $\mathrm{S}_{1} \rightarrow \mathrm{S}_{0}$ of 3. During the electronic transition, the electron density increases in the yellow areas and decreases in the blue areas.

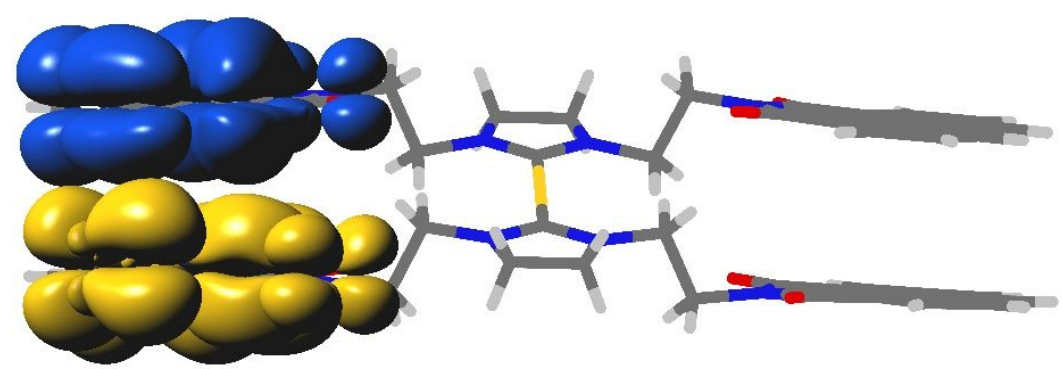

Figure S50. PBE0-DFT transition densities for $\mathrm{S}_{1} \rightarrow \mathrm{S}_{0}$ of 4 . During the electronic transition, the electron density increases in the yellow areas and decreases in the blue areas. 


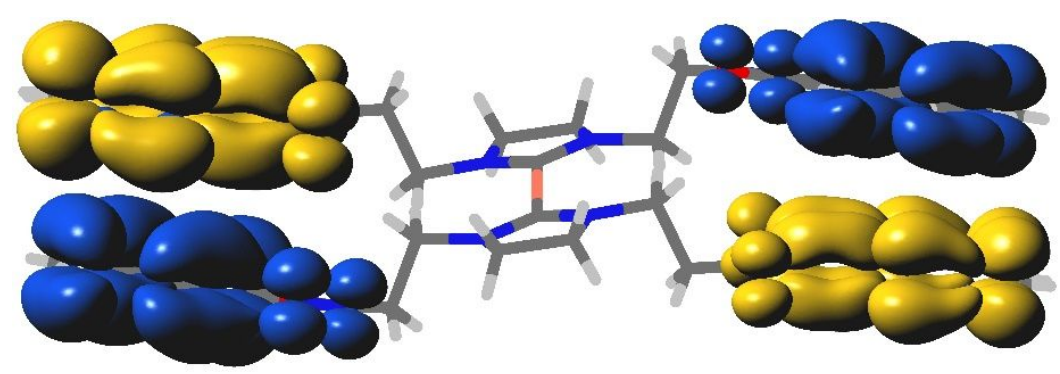

Figure S51. PBE0-DFT transition densities for $\mathrm{S}_{1} \rightarrow \mathrm{S}_{0}$ of 5. During the electronic transition, the electron density increases in the yellow areas and decreases in the blue areas.

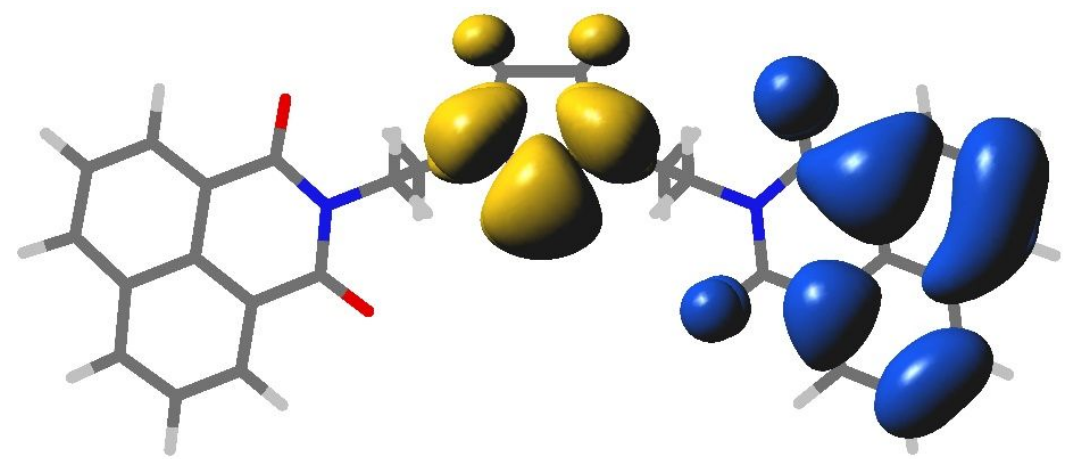

Figure S52. PBE0-DFT transition densities for $\mathrm{S}_{1} \rightarrow \mathrm{S}_{0}$ of 2a. During the electronic transition, the electron density increases in the yellow areas and decreases in the blue areas.

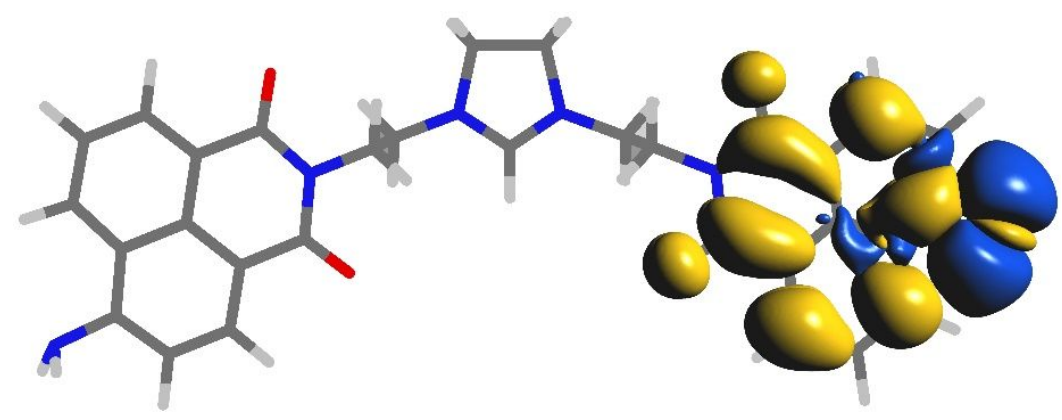

Figure S53. PBE0-DFT transition densities for $\mathrm{S}_{1} \rightarrow \mathrm{S}_{0}$ of 2c. During the electronic transition, the electron density increases in the yellow areas and decreases in the blue areas.

Table S6. Changing Procedure of Transition Dipole Moment (Debye) via Angular displacement of on NMI relative to another

\begin{tabular}{llll}
\hline $\begin{array}{l}\text { NMI...NMI } \\
\text { Angle } \\
\text { (Degree) }\end{array}$ & $\begin{array}{l}\text { Angular } \\
\text { (Debye) }\end{array}$ & $\begin{array}{l}\text { Angular } \\
\text { (Debye) }\end{array}$ & $\begin{array}{l}\text { Angle } \\
\text { (Degree) }\end{array}$ \\
\hline 90 & 5.34324 & 6.33452 & 90 \\
80 & 5.69413 & 6.12586 & 80 \\
70 & 6.16707 & 7.00048 & 70 \\
66 & 6.25482 & 7.15831 & 66 \\
62 & 6.65482 & 7.39832 & 62 \\
58 & 6.74994 & 7.54624 & 58 \\
54 & 6.85237 & 7.79879 & 54
\end{tabular}




\begin{tabular}{llll}
48 & 6.90077 & 7.89015 & 48 \\
44 & 7.01655 & 8.10541 & 44 \\
40 & 7.1249 & 8.83294 & 40 \\
38 & 7.38524 & 9.10972 & 38 \\
36 & 7.22012 & 8.65912 & 36 \\
34 & 6.4622 & 7.9646 & 34 \\
32 & 6.31067 & 7.75722 & 32 \\
30 & 6.15775 & 7.6572 & 30 \\
28 & 5.99645 & 7.55943 & 28 \\
26 & 5.82475 & 7.45312 & 26 \\
24 & 5.64894 & 7.34065 & 24 \\
22 & 5.46992 & 7.22252 & 22 \\
20 & 5.283 & 7.09722 & 20 \\
18 & 5.08976 & 6.96256 & 18 \\
16 & 4.90399 & 6.81766 & 16 \\
14 & 4.75248 & 6.66412 & 14 \\
12 & 4.63227 & 6.50137 & 12 \\
10 & 4.50245 & 6.32684 & 10 \\
8 & 4.32486 & 6.13948 & 8 \\
6 & 4.10048 & 6.01054 & 6 \\
4 & 3.86167 & 5.99642 & 4 \\
2 & 3.6818 & 5.81517 & \\
\hline
\end{tabular}

Table S7. Compound 3; orbitals contribution $\mathrm{S} 1 \rightarrow \mathrm{S} 0$ (Gas State)

\begin{tabular}{ccccc} 
No. & From & To & \multicolumn{2}{c}{ Contribution } \\
\hline 1 & HOMO - 4 & LUMO & 0.017159 & $1.7 \%$ \\
2 & HOMO - 3 & LUMO & 0.216893 & $21.7 \%$ \\
3 & HOMO - 3 & LUMO + 2 & 0.012952 & $1.3 \%$ \\
4 & HOMO - 2 & LUMO & 0.342697 & $34.3 \%$ \\
5 & HOMO - 2 & LUMO + 2 & 0.017915 & $1.8 \%$ \\
6 & HOMO - 1 & LUMO & 0.357247 & $35.7 \%$ \\
7 & HOMO - 1 & LUMO + 2 & 0.01426 & $1.4 \%$ \\
\hline
\end{tabular}

Table S8. Compound 4; orbitals contribution $\mathrm{S} 1 \rightarrow \mathrm{S} 0$ (Gas State) (Linear)

\begin{tabular}{ccccc} 
No. & From & To & \multicolumn{2}{c}{ Contribution } \\
\hline 1 & HOMO -5 & LUMO & 0.075701 & $7.5 \%$ \\
2 & HOMO -5 & LUMO + 2 & 0.073115 & $7.3 \%$ \\
3 & HOMO -4 & LUMO + 1 & 0.084393 & $8.4 \%$ \\
4 & HOMO -4 & LUMO + 3 & 0.080744 & $8.0 \%$ \\
5 & HOMO - 2 & LUMO & 0.044764 & $4.5 \%$ \\
6 & HOMO - 1 & LUMO + 1 & 0.305713 & $30.5 \%$
\end{tabular}




\begin{tabular}{ccccc}
7 & HOMO & LUMO & 0.252817 & $25.3 \%$ \\
\hline \multicolumn{5}{c}{ Table S9. Compound 5; orbitals contribution S1 $\rightarrow$ S0 (Gas State) } \\
No. & From & To & Contribution \\
\hline 1 & HOMO - 3 & LUMO & 0.016852 & $1.7 \%$ \\
2 & HOMO - 2 & LUMO + 1 & 0.015439 & $1.5 \%$ \\
3 & HOMO - 1 & LUMO + 1 & 0.388318 & $39.0 \%$ \\
4 & HOMO - 1 & LUMO + 2 & 0.014942 & $1.5 \%$ \\
5 & HOMO - 1 & LUMO + 3 & 0.05864 & $6 \%$ \\
6 & HOMO & LUMO & 0.402401 & $40.2 \%$ \\
7 & HOMO & LUMO + 2 & 0.072387 & $7.2 \%$ \\
\hline
\end{tabular}

Table S10. Compound 6; orbitals contribution $\mathrm{S} 1 \rightarrow \mathrm{S} 0$ (Gas State)

\begin{tabular}{lllll} 
No. & From & To & Contribution & \\
\hline 1 & HOMO & LUMO & 0.997995 & $99.7 \%$ \\
\hline
\end{tabular}

Table S11. Ag-NHC-NH-Nap orbitals contribution S1-S0 (Gas State)

\begin{tabular}{ccccc} 
No. & From & To & \multicolumn{2}{c}{ Contribution } \\
\hline 1 & HOMO & LUMO & 0.985322 & $98.5 \%$ \\
\hline
\end{tabular}

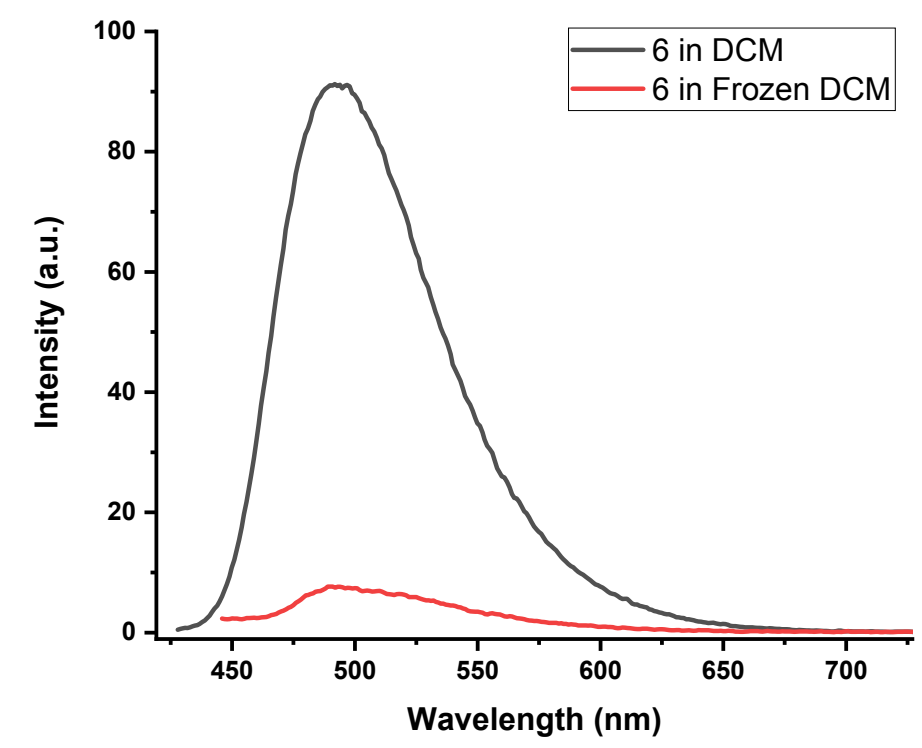

Figure S54. Emission spectra of 6 in DCM (black) and frozen DCM (red) 


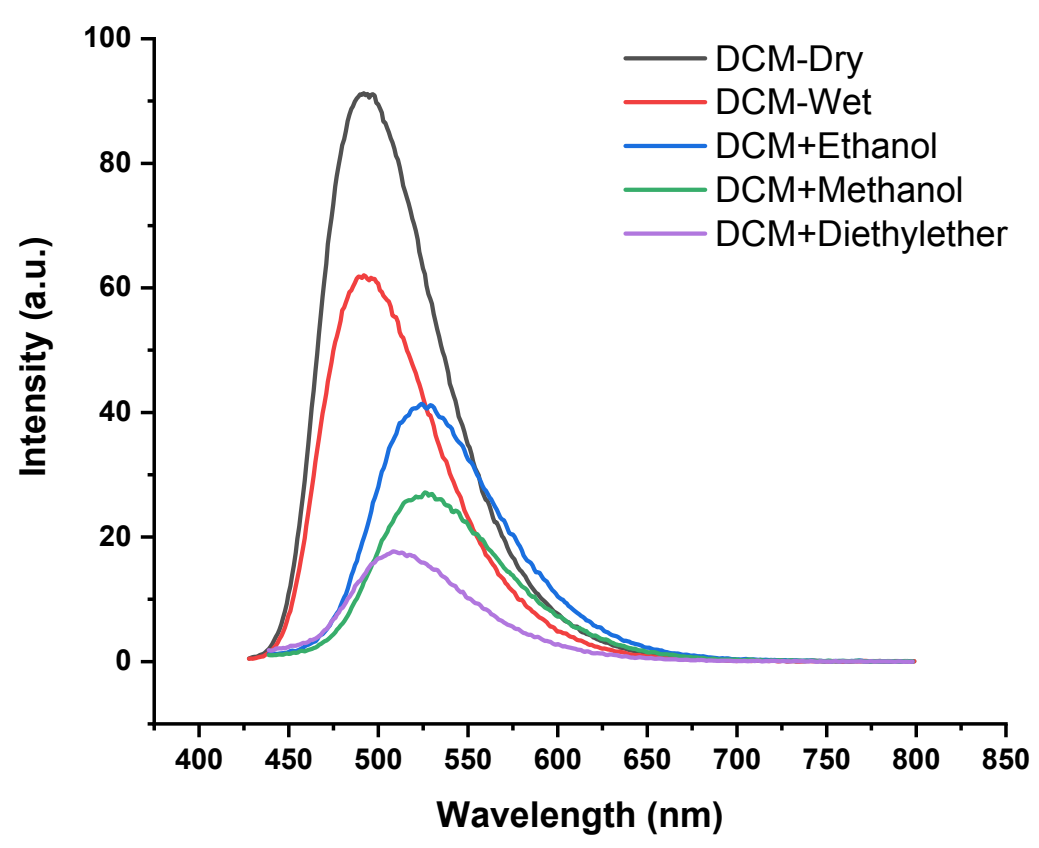

Figure S55. Emission spectra of 6 in dry DCM and DCM + H-bonding solvents

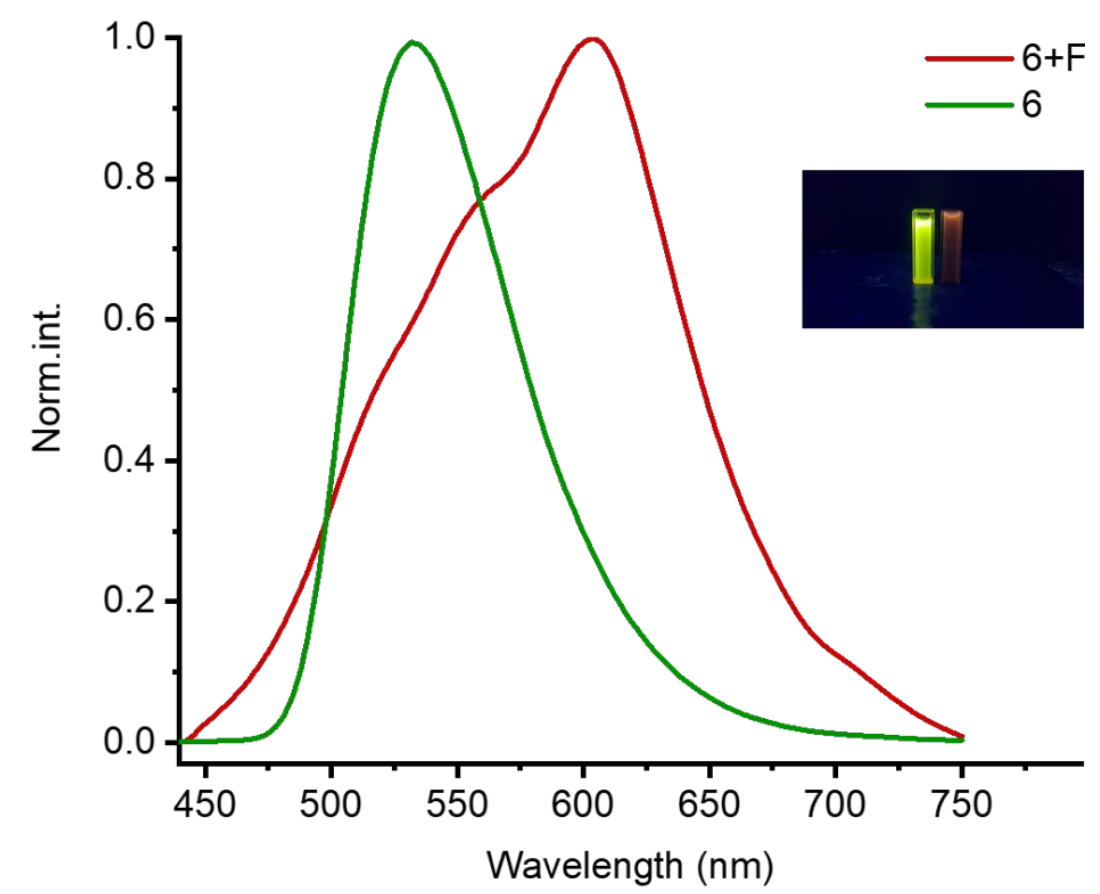

Figure S56. The emission spectra of 6 in dmso solution before (green line) and after (red line) addition of Tetrabuthylammonium fluoride 


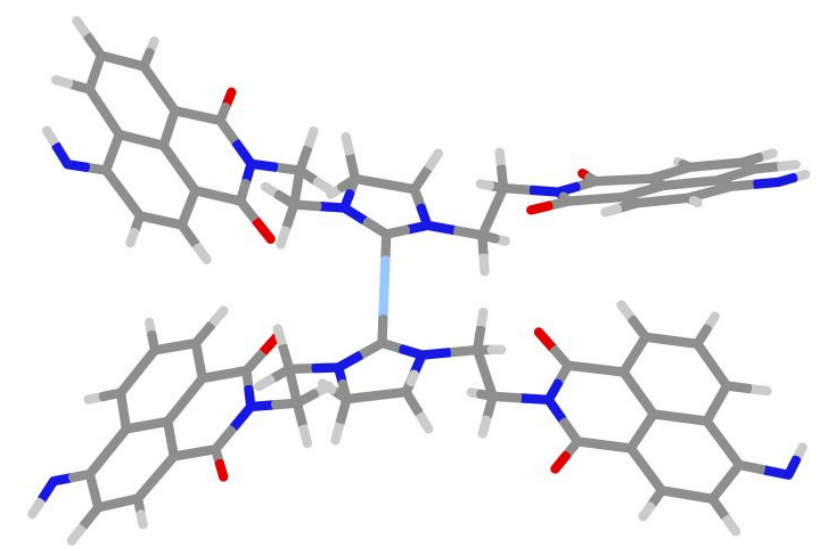

Figure S57. Molecular view of optimized geometries of deprotonated 6.

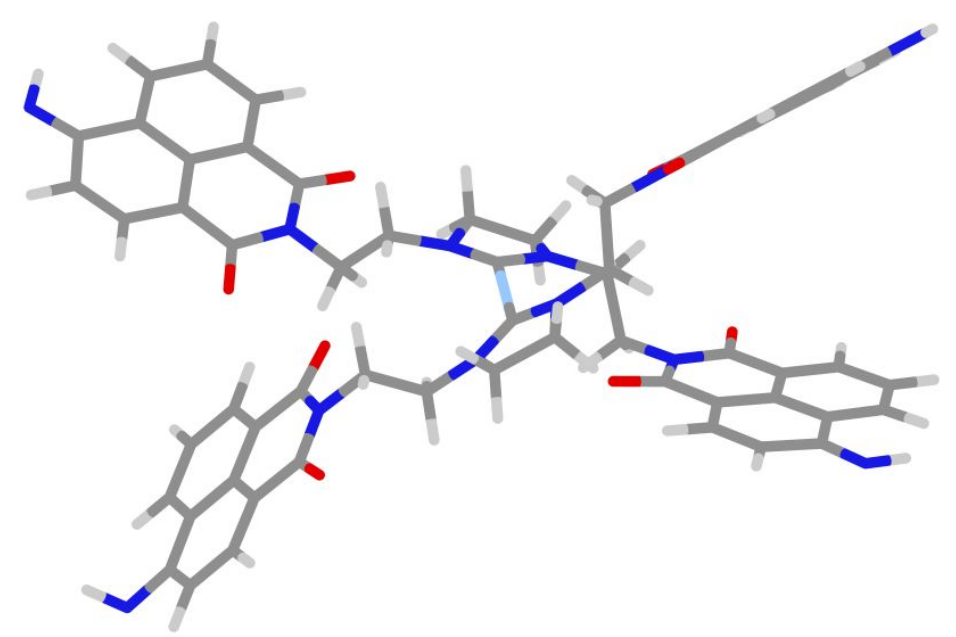

Figure S58. Molecular view of optimized geometries of deprotonated 6 in First Excited-State $\left(\mathrm{S}_{1}\right)$.

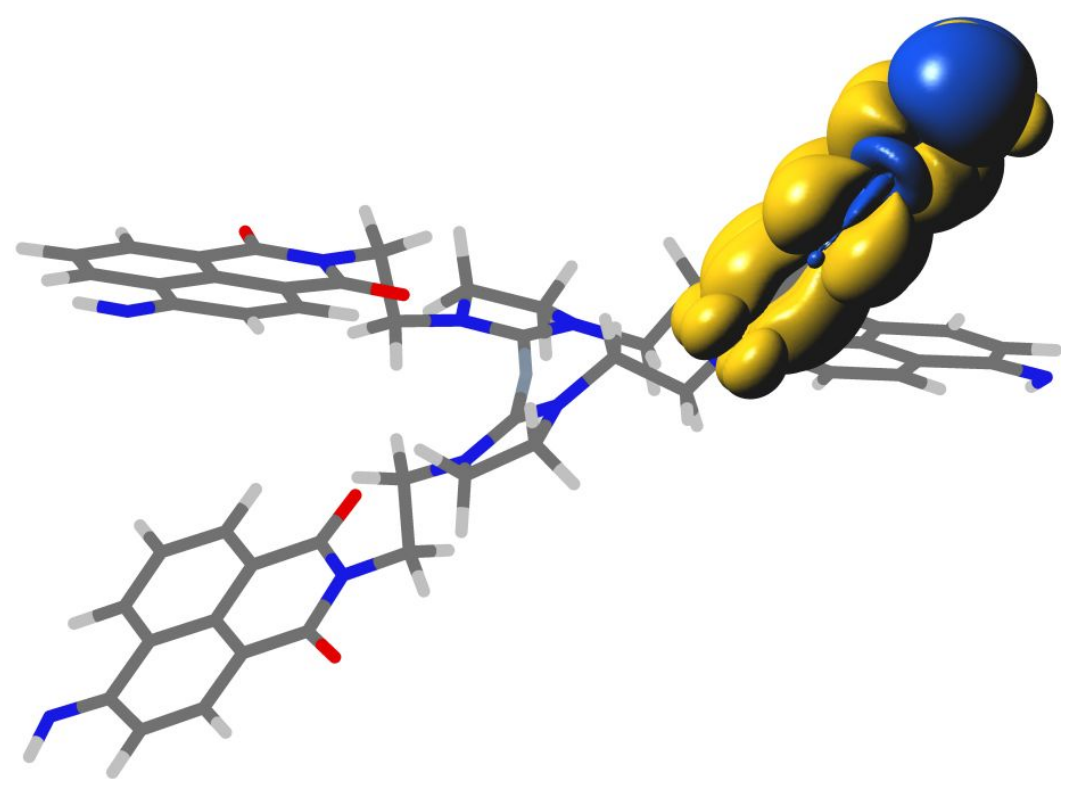

Figure S59. PBE0-DFT transition densities for $\mathrm{S}_{1} \rightarrow \mathrm{S}_{0}$ of deprotonated 6. During the electronic transition, the electron density increases in the yellow areas and decreases in the blue areas. 


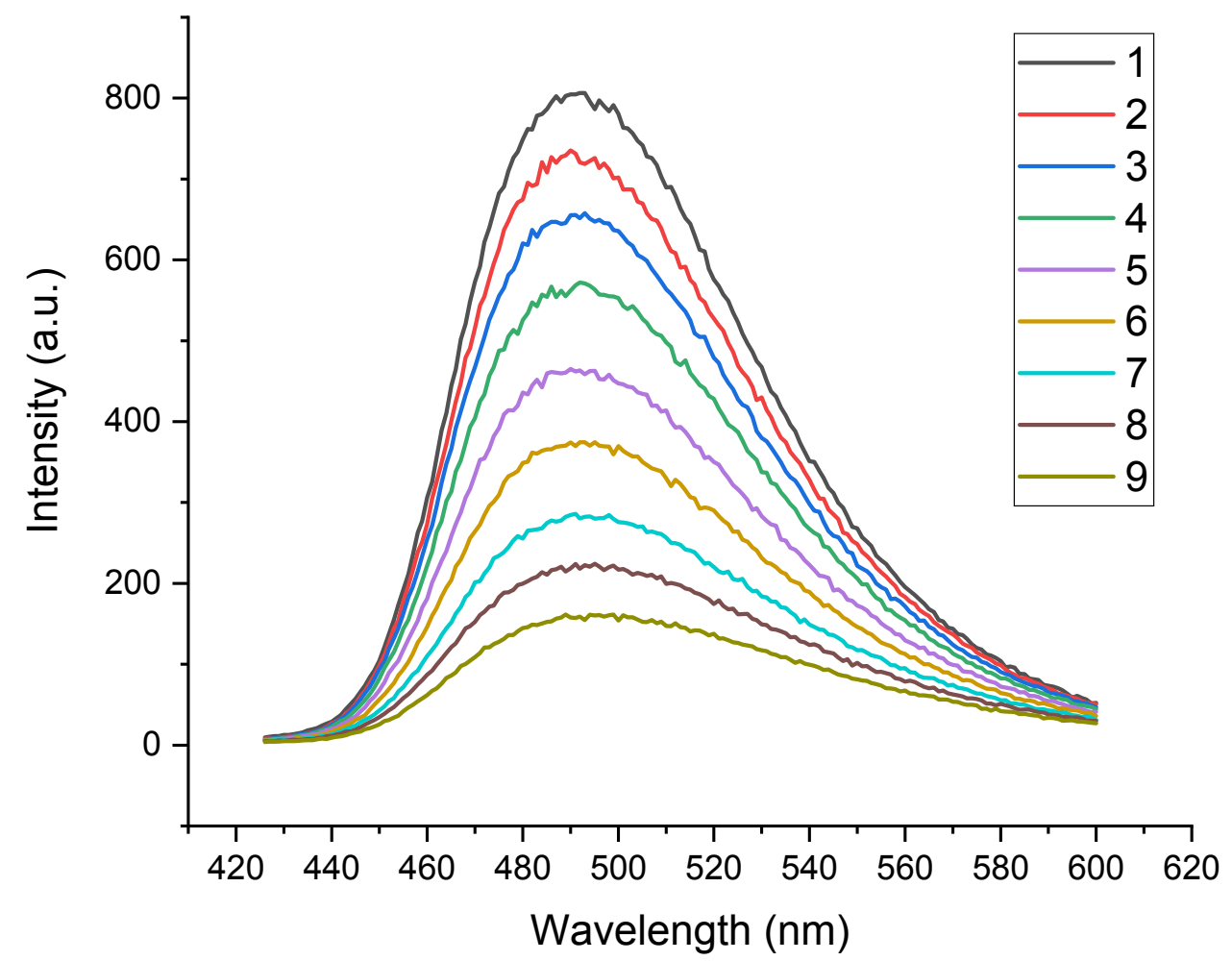

Figure S60. Quenching of excimeric emission of 6 in the presence of fluoride ion. 


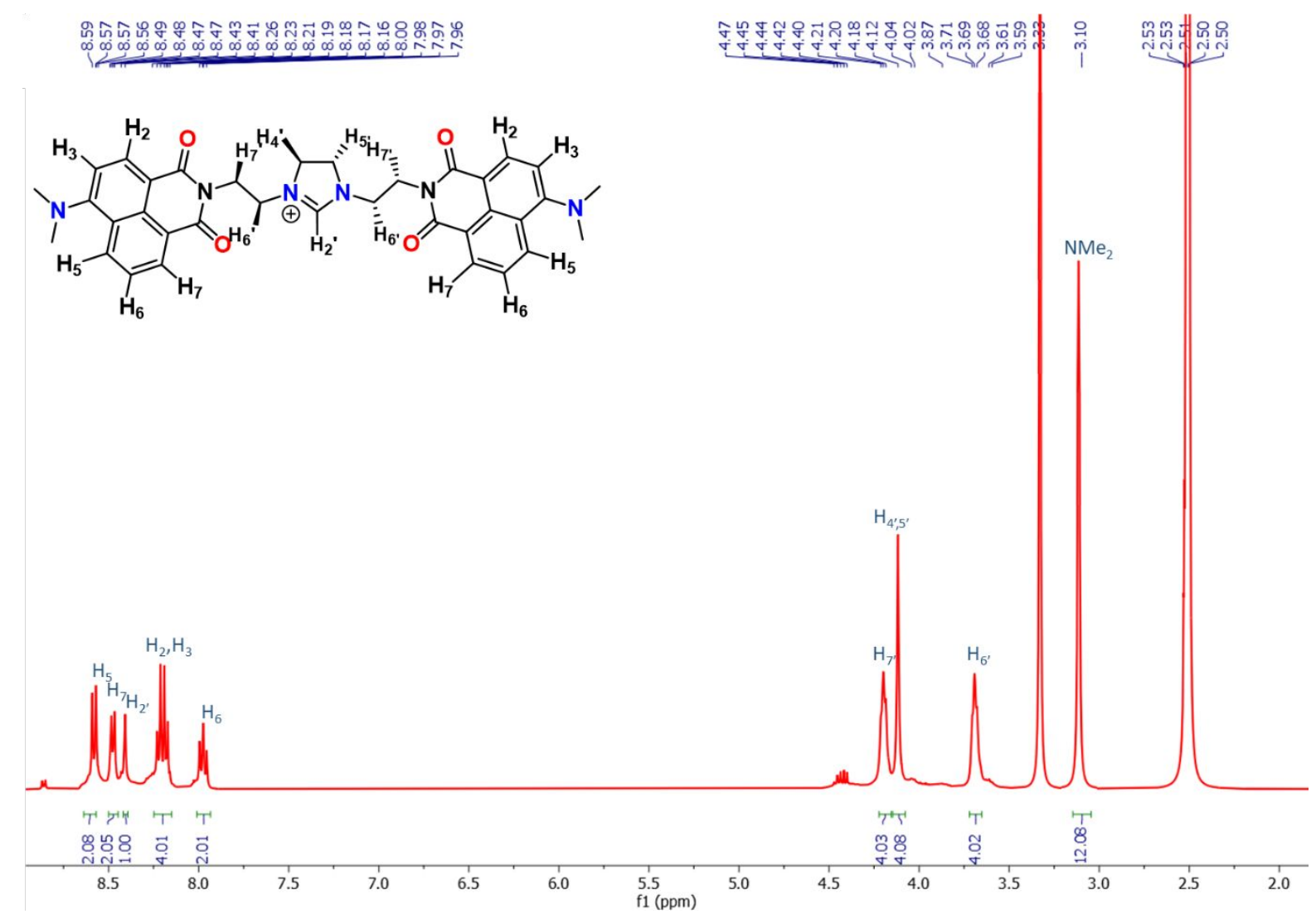

Figure S61. ${ }^{1} \mathrm{H}-\mathrm{NMR}$ spectrum of 4-dimethylamine derivative of 2a in DMSO- $d_{6}$ at $298 \mathrm{~K}$.

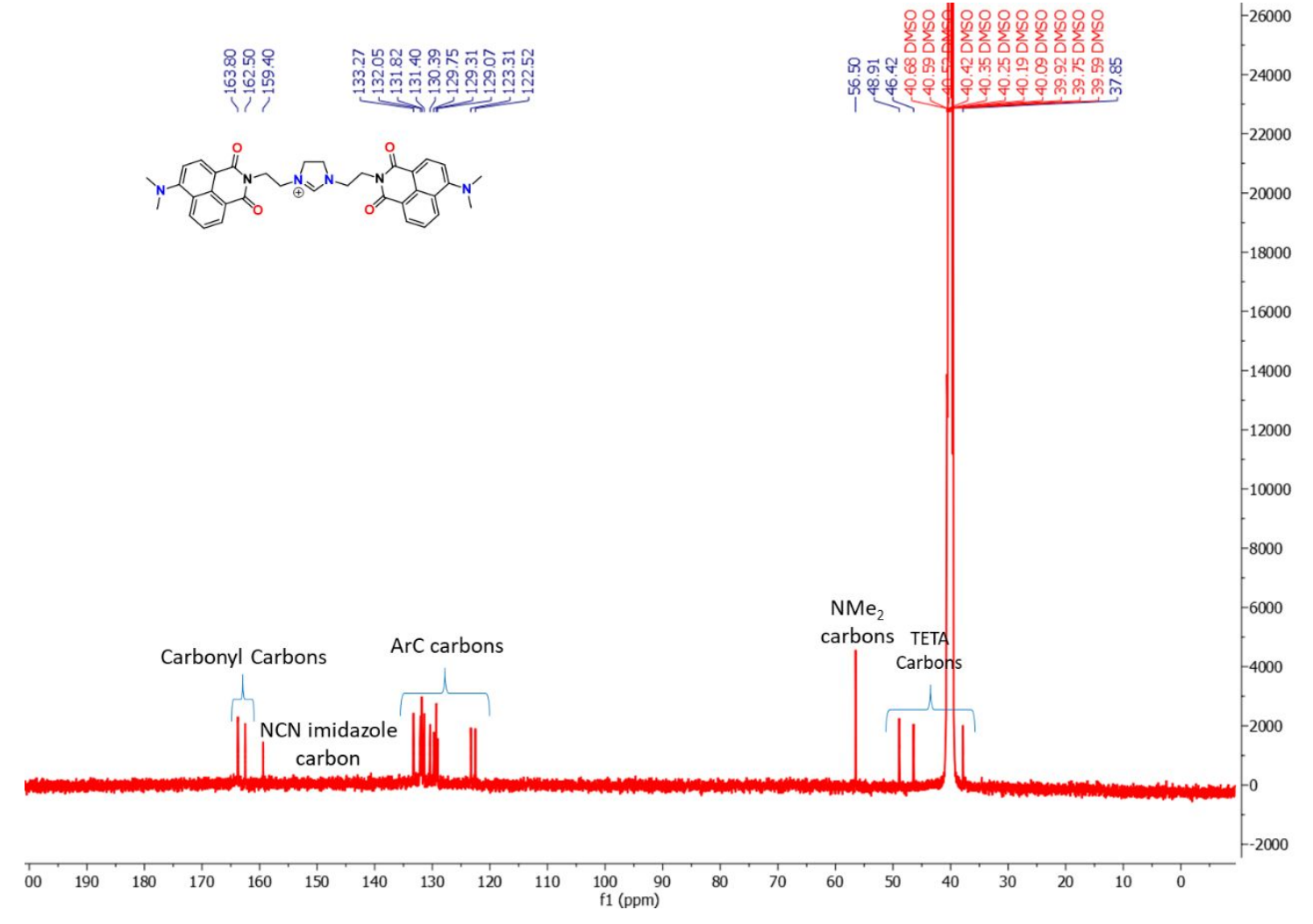

Figure S62. ${ }^{13} \mathrm{C}\left\{{ }^{1} \mathrm{H}\right\}$ NMR spectrum of 4-dimethylamine derivative of $\mathbf{2 a}$ in DMSO- $d_{6}$ at $298 \mathrm{~K}$. 


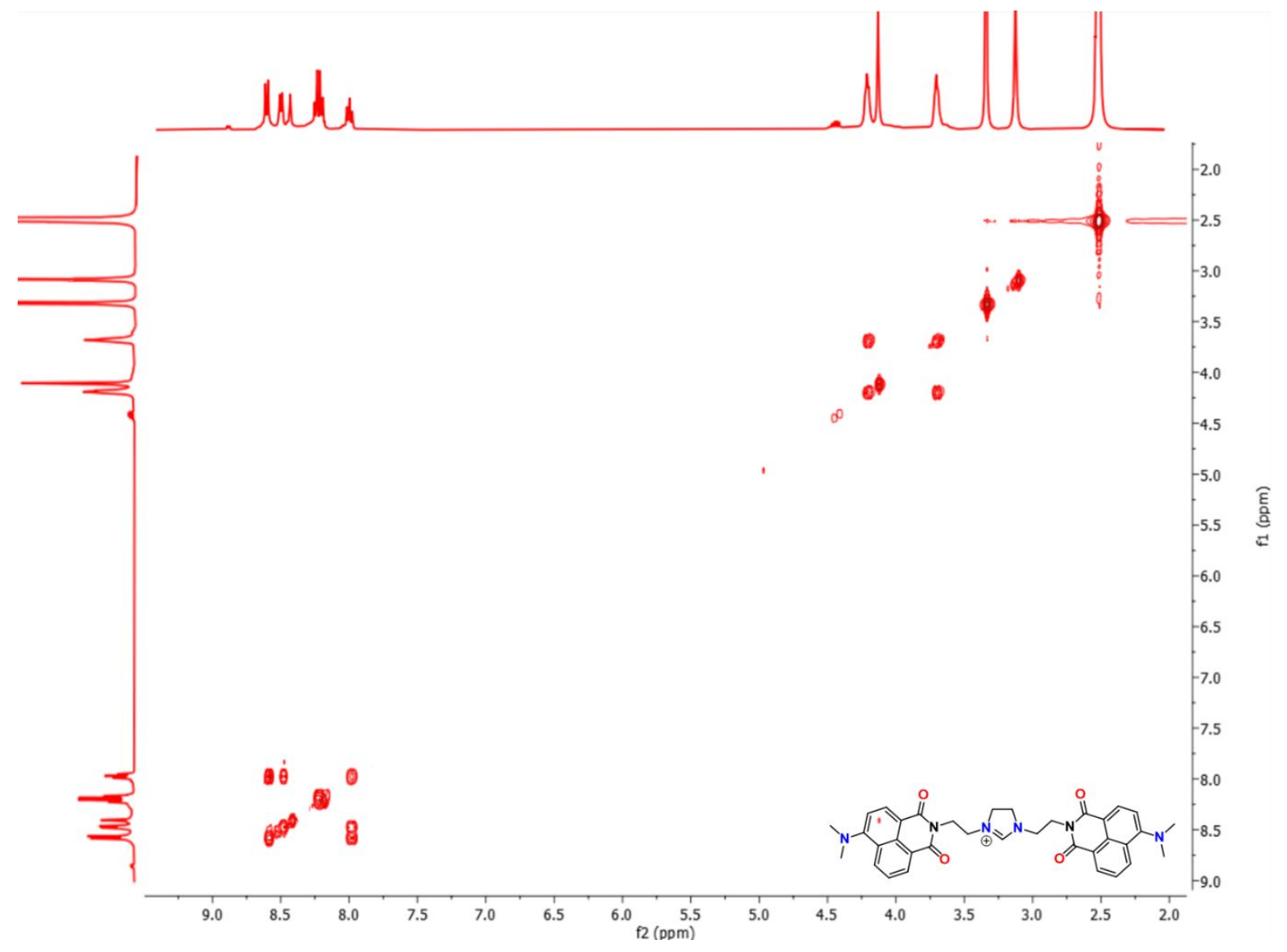

Figure S63. ${ }^{1} \mathrm{H}-{ }^{13} \mathrm{C}$ HSQC NMR spectrum of 4-dimethylamine derivative of 2a in DMSO- $d_{6}$ at $298 \mathrm{~K}$.

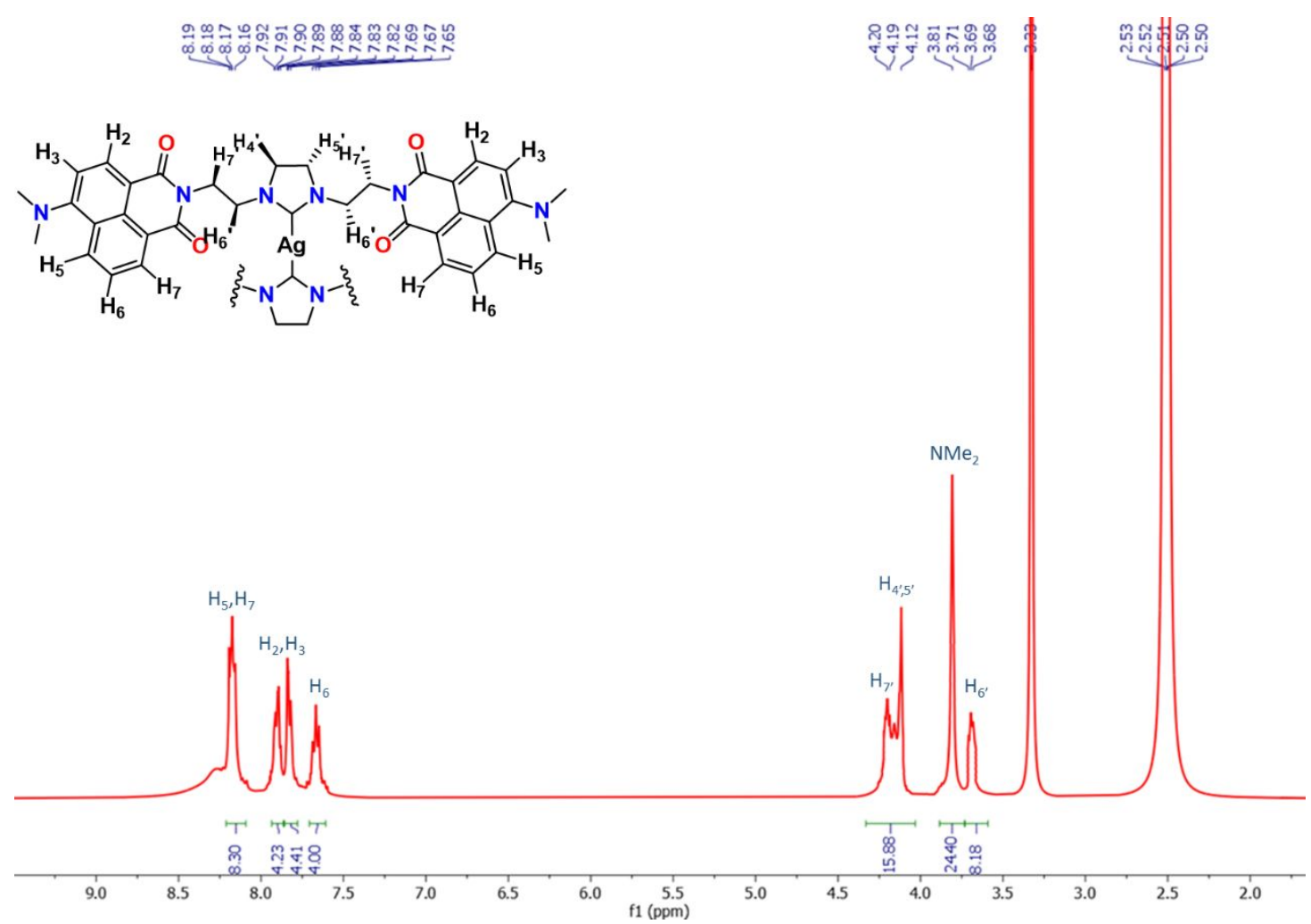

Figure S64. ${ }^{1} \mathrm{H}-\mathrm{NMR}$ spectrum of 4-dimethylamine derivative of 3 in DMSO- $d_{6}$ at $298 \mathrm{~K}$. 

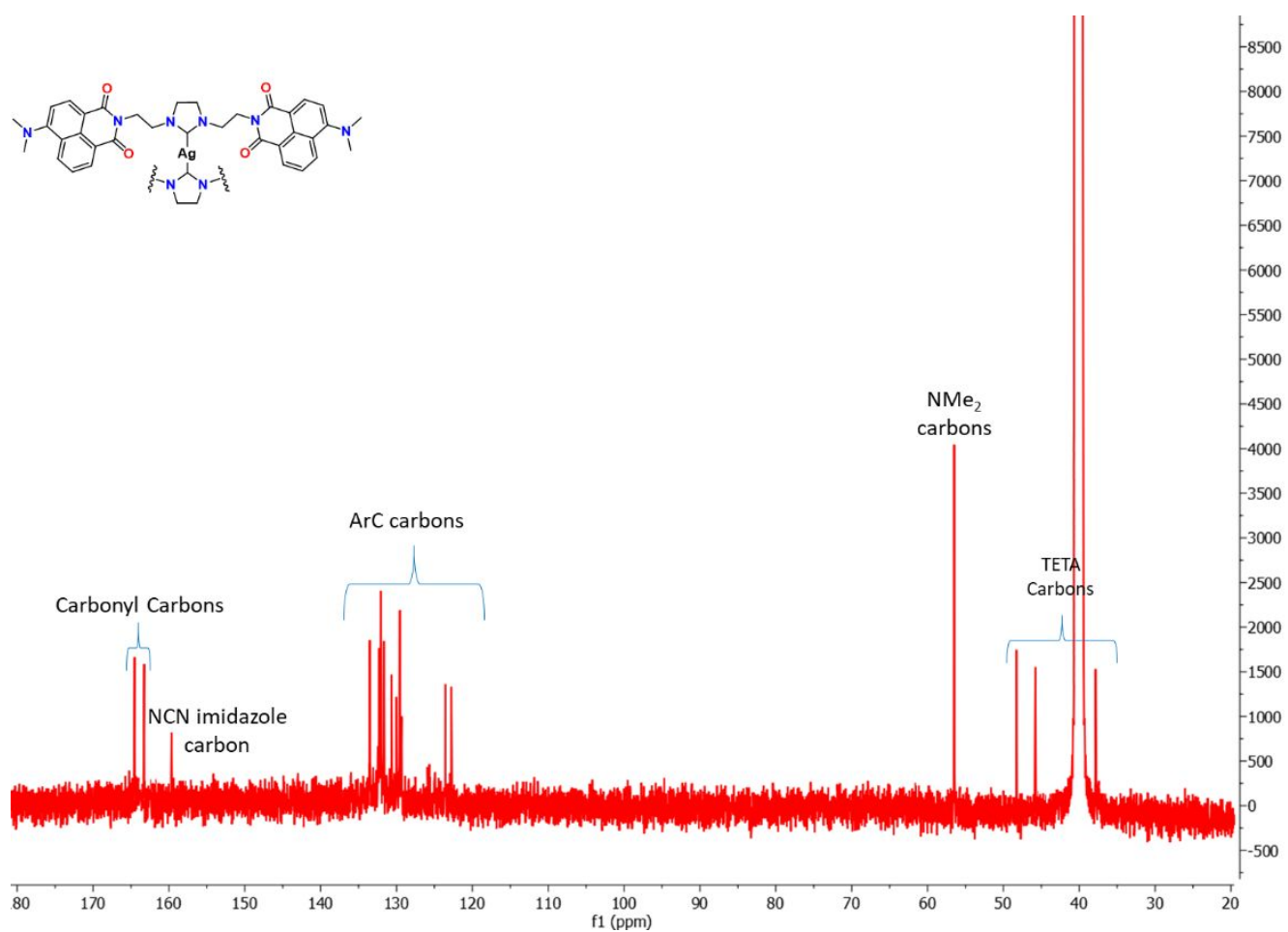

Figure S65. ${ }^{13} \mathrm{C}\left\{{ }^{1} \mathrm{H}\right\}$ NMR spectrum of 4-dimethylamine derivative of $\mathbf{3}$ in DMSO- $d_{6}$ at $298 \mathrm{~K}$

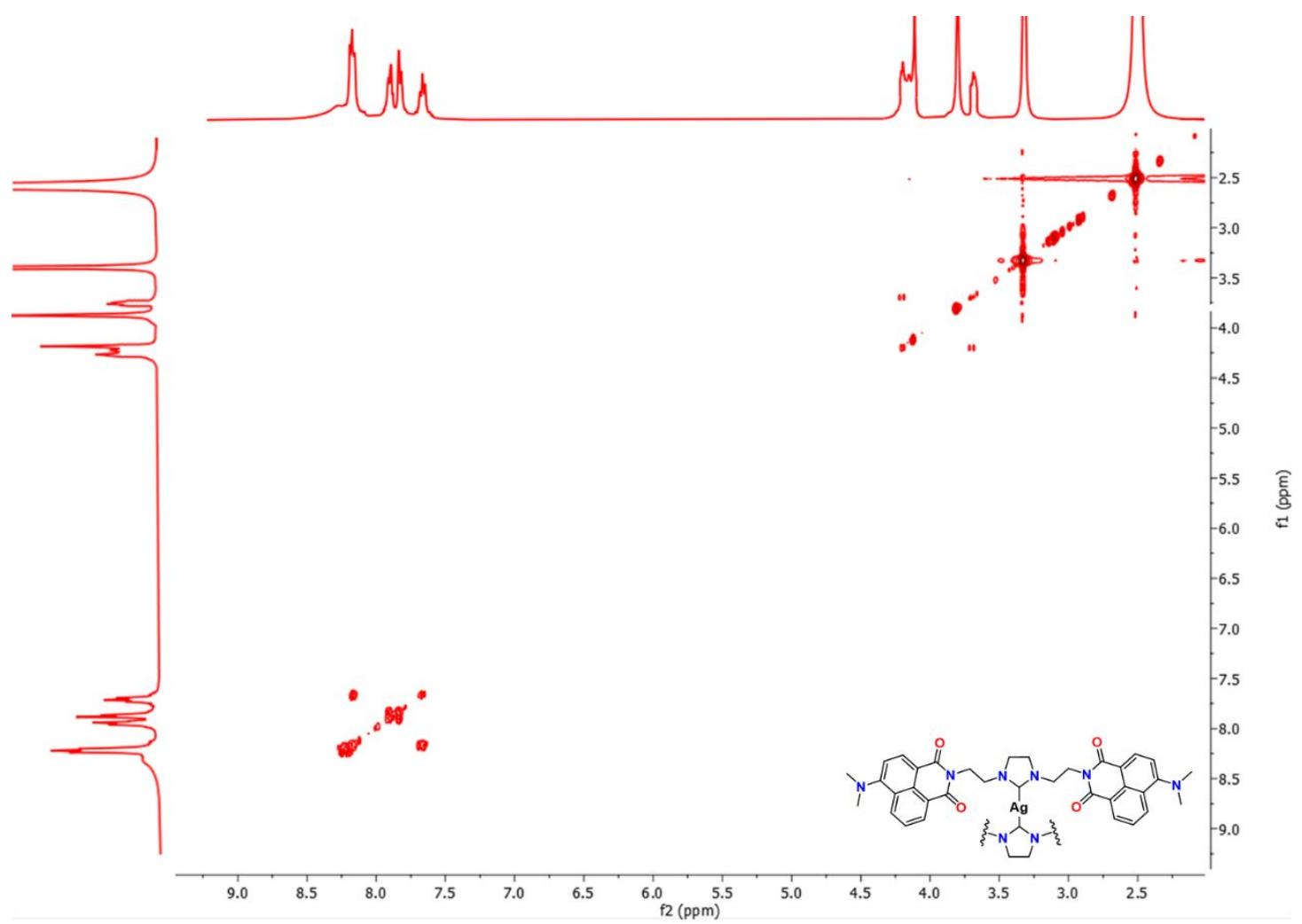

Figure S63. ${ }^{1} \mathrm{H}^{-13} \mathrm{C}$ HSQC NMR spectrum of 4-dimethylamine derivative of 2a in DMSO- $d_{6}$ at $298 \mathrm{~K}$. 


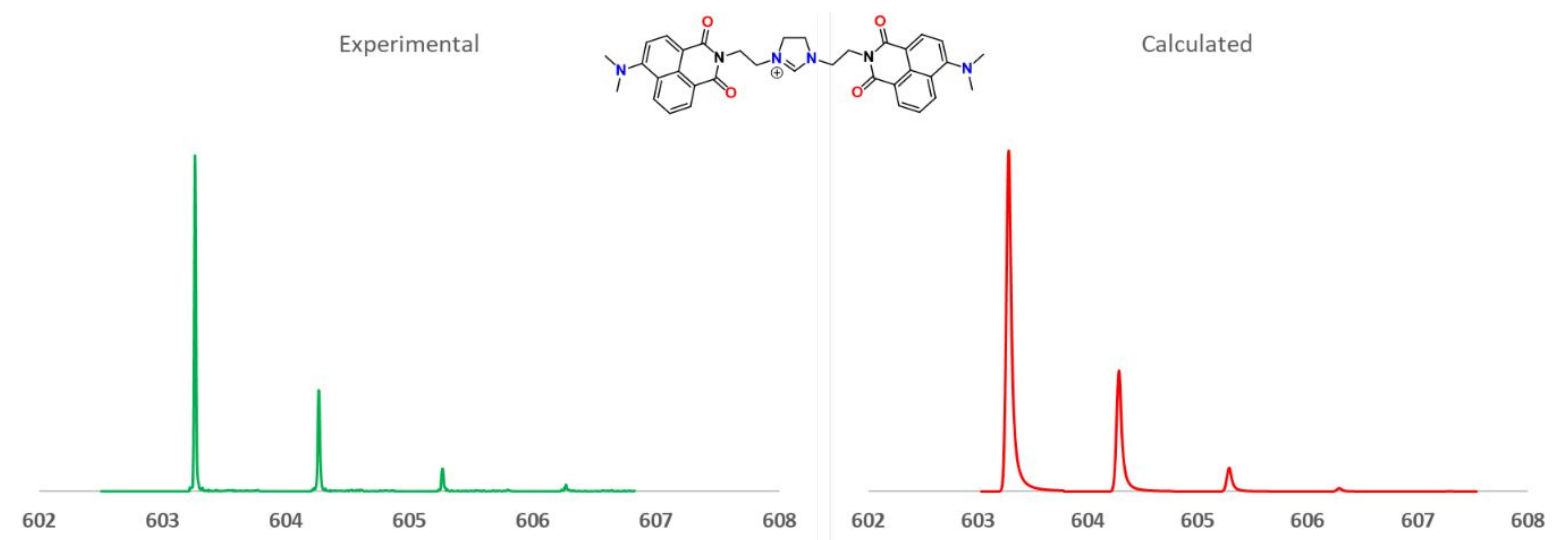

Figure S67. ESI $^{+}$HRMS spectrum of 4-dimethylamine derivative of 2a. (green) Expansion of the dicationic fragment signal $\left[\mathrm{C}_{35} \mathrm{H}_{39} \mathrm{~N}_{6} \mathrm{O}_{4}\right]^{+}$at $\mathrm{m} / \mathrm{z}=603$, (red) simulated isotopic pattern of the cationic fragment $\left[\mathrm{C}_{35} \mathrm{H}_{35} \mathrm{~N}_{6} \mathrm{O}_{4}\right]^{+}$

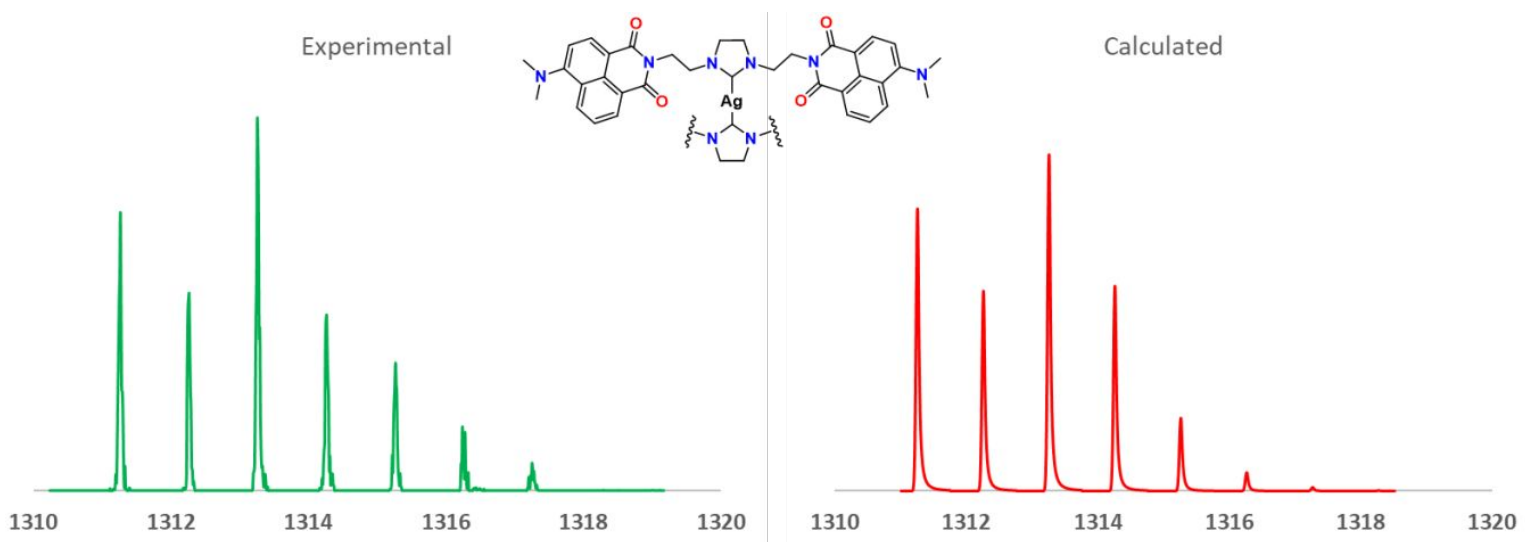

Figure S68. ESI $^{+}$HRMS spectrum of 4-dimethylamine derivative of 3. (green) Expansion of the dicationic fragment signal $\left[\mathrm{C}_{70} \mathrm{H}_{72} \mathrm{AgN}_{12} \mathrm{O}_{8}\right]^{+}$at $\mathrm{m} / \mathrm{z}=1313$, (red) simulated isotopic pattern of the cationic fragment $\left[\mathrm{C}_{70} \mathrm{H}_{70} \mathrm{AgN}_{12} \mathrm{O}_{8}\right]^{+}$

Table S12. Photophysical data in dichloromethane solution and solid state

\begin{tabular}{ccccc}
\hline Compound & $\lambda_{\mathrm{em}}{ }^{\mathrm{a}}(\mathrm{nm})$ solution/solid & $\lambda_{\mathrm{ex}}(\mathrm{nm})$ & $\mathrm{T}^{\mathrm{b}}(\mathrm{ns})$ solution/solid & $\Phi_{\mathrm{F}}{ }^{\mathrm{c}}$ solution/solid \\
\hline $\begin{array}{c}\text { 4-dimethylamine } \\
\text { derivative of 2a }\end{array}$ & $532 / 566$ & 432 & $10.64 / 7.51$ & $36.6 / 0.34$ \\
$\begin{array}{c}\text { 4-dimethylamine } \\
\text { derivative of 3 }\end{array}$ & $520 / 586$ & 432 & $28.18 / 23.55$ & $27.8 / 0.21$ \\
\hline
\end{tabular}

aMaximum wavelength of fluorescence emission spectra. ${ }^{b}$ Fluorescence lifetimes for emission in the maximum wavelength of fluorescence emission. ${ }^{c}$ Fluorescence quantum efficiency. 


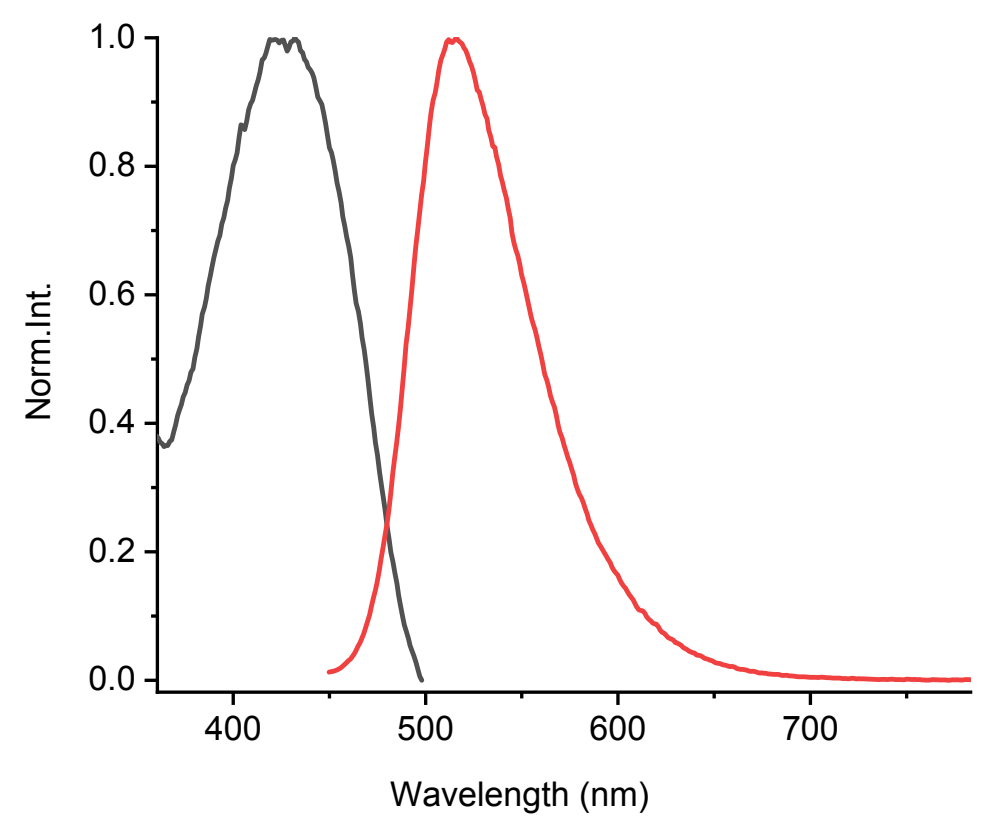

Figure S69. Excitation (black) and emission (red) spectra of 4-dimethylamine derivative of 3.

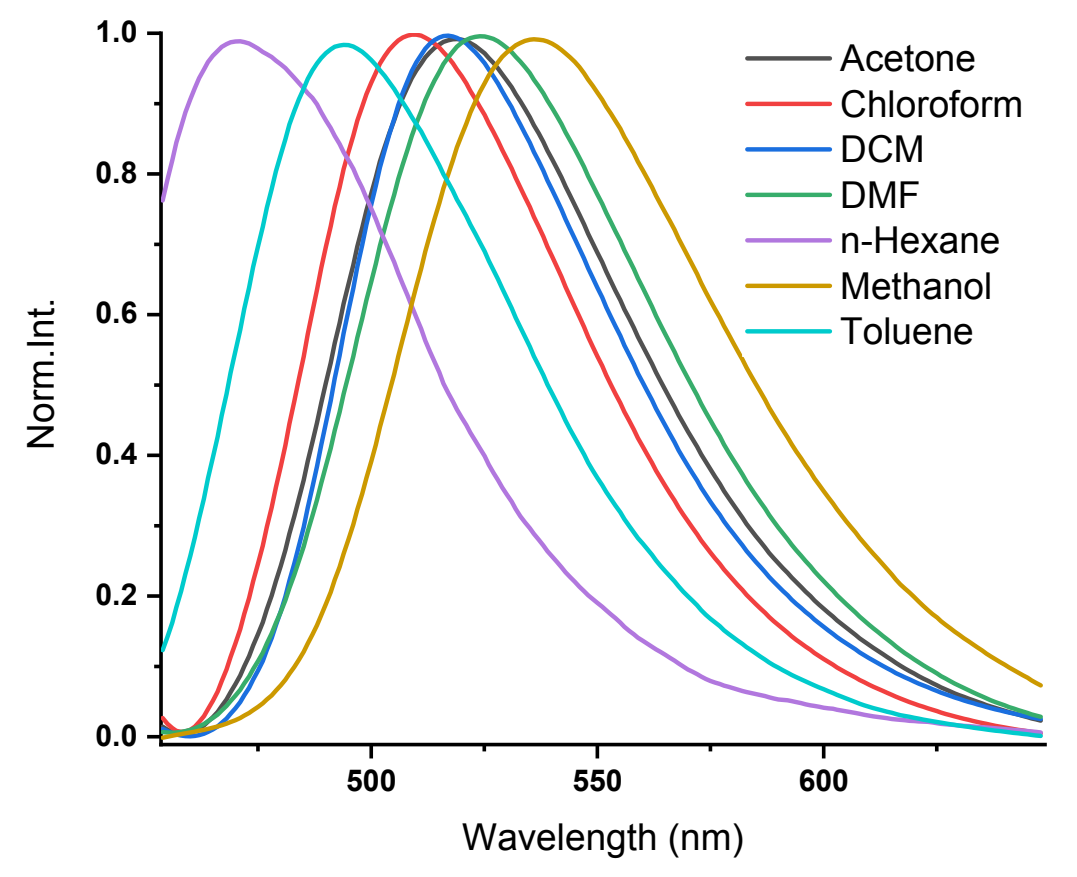

Figure S70. Emission spectra of 4-dimethylamine derivative of $\mathbf{3}$ in different solvents. 


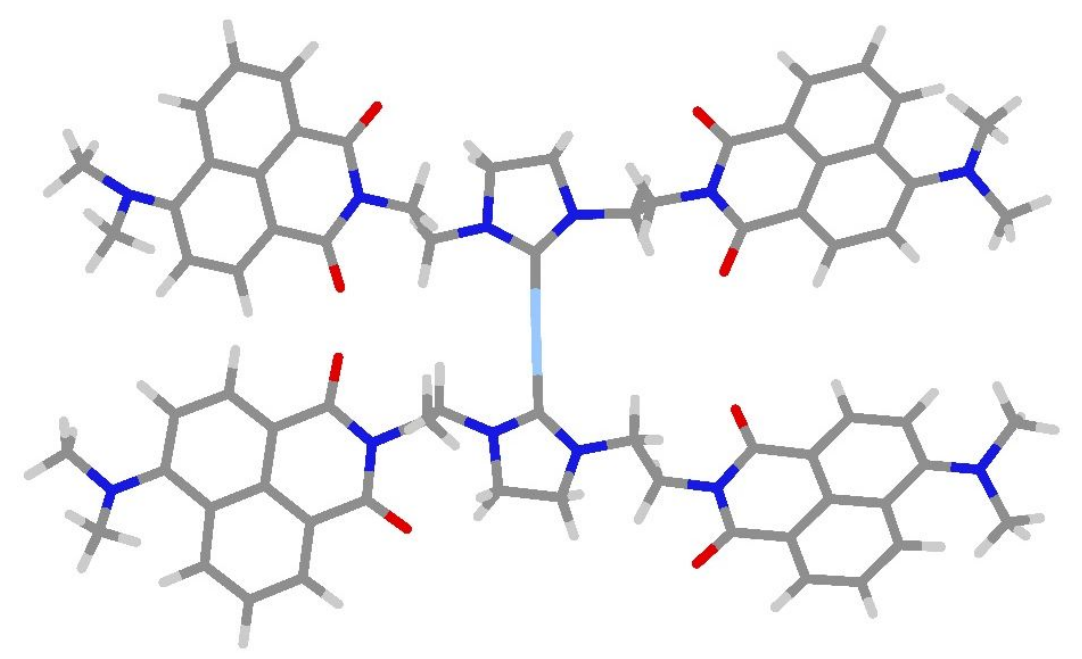

Figure S71. The optimized structure of 4-dimethylamine derivative of $\mathbf{3}$ in ground state.

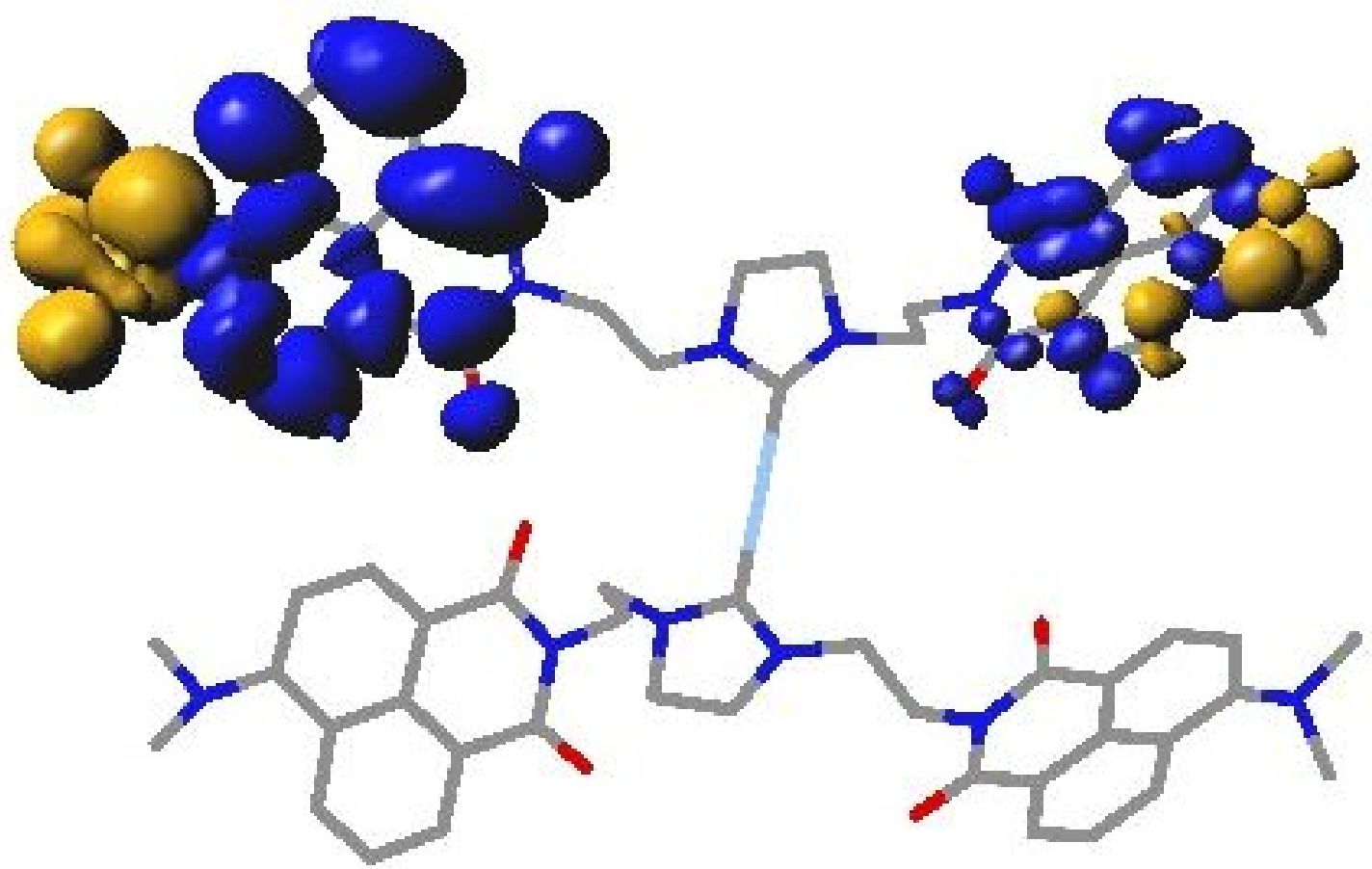

Figure S72. Transition density map of 4-dimethylamine derivative of $\mathbf{3}$. 


\section{References}

(1) Neese, F. Software update: the ORCA program system, version 4.0. WIREs Comput. Mol. Sci. 2018, 8, e1327.

(2) Clark, R. C.; Reid, J. S. The analytical calculation of absorption in multifaceted crystals. Acta Crystallogr., Sect. A: Found. Crystallogr. 1995, 51, 887-897.

(3) SuperNova Eos S2 System: Empirical absorption correction, 2011, Crys Alis-Software package; Oxford Diffraction Ltd.

(4) Dolomanov, O. V.; Bourhis, L. J.; Gildea, R. J.; Howard, J. A. K.; Puschmann, H. OLEX2: a complete structure solution, refinement and analysis program. J. Appl. Crystallogr. 2009, 42, 339-341. Auto Chem. 2.0, in conjunction with OLEX2; Agilent Technologies UK Ltd.: Yarnton, England, 2012.

(5) Spek, A. L. Platon: A Multipurpose Crystallographic Tool, version 1.17; Utrecht University: Utrecht, The Netherlands, 2013. Sheldrick, G. M. Acta Crystallogr., Sect. A: Found. Crystallogr. 2008, A64, 112- 122.

(6) Spek, A. L, Structure validation in chemical crystallography. Acta Crystallogr., Sect. D: Biol. Crystallogr. 2009, D65, 148-155.

(7) Viji, M.; Nair, A. K.; Nandajan P. C.; Ramaiah. D., Fluorescent chemodosimeter based on NHC complex for selective recognition of cyanide ions in aqueous medium. RSC Adv., 2014, 4, 47982. 